K. Yamato

Nagoya Math. J

Vol. 76 (1979), 35-114

\title{
AN EFFECTIVE METHOD OF COUNTING THE NUMBER OF LIMIT CYCLES
}

\author{
KAZUO YAMATO
}

\section{Introduction}

We are interested in determining, after a finite number of procedures, the number and the approximate positions of limit cycles for a given system.

For instance, let

$$
\text { (*) } \quad\left\{\begin{array}{l}
\dot{x}=f(x, y) \\
\dot{y}=g(x, y)
\end{array}\right.
$$

be a given autonomous system on an $(x, y)$-plane $\boldsymbol{R}^{2}$. Suppose that analytic expressions for the solutions of $(*)$ are not to be expected. Then in order to know the analytic properties of the solutions, we have to study the "pattern" described by the family of integral curves. For this purpose it is of basic importance to investigate the singular points (points such that $f(x, y)=g(x, y)=0$ ) and the limit cycles (isolated closed integral curves), because the singular points and the limit cycles dominate the global pattern. The problem of investigating the singular points is the one of algebraic equations, while as to the problem of investigating the limit cycles, any effective, general method has not been known yet.

The purpose of the present paper is to give a method by which the number and the approximate positions of limit cycles can be determined. Indeed we shall show that

(i) our problem (i.e. determining the number and the approximate positions of limit cycles) can be reduced to the problem of finding approximate solutions of a partial differential equation (denoted by $(\mathrm{E})$ below),

and as applications of our method to Liénard's equations we shall prove (ii) the classical theorem concerning the generating circles of limit Received October 3, 1978. 
cycles (the typical result by the classical perturbation technique).

(iii) the Liénard's theorem (the existence of a unique limit cycle).

(iv) the comparatively recent result (Hochstadt, Stephan and D'heedene) concerning the existence of an infinite number of limit cycles.

As for the features of our method we wish to emphasize the following:

(i) In our proof of the existence of limit cycles we have not to draw any geometrical figures. Moreover, the approximate positions of limit cycles are given by algebraic inequalities. In this sense, our theory plays the same role in the problems of limit cycles as analytic geometry plays in purely geometric problems.

(ii) The essential part of our existence-proof of limit cycles is to find the function by which the given system is to be multiplied. Therefore, after having found it, we can note in a small space the function as the key to the proof.

(iii) Because of the differential-topological property of our method, the function (mentioned above) can be used also in the study of the perturbed systems (and hence in the study of the forced systems).

Let us explain our method in more detail. Let $X$ be the vector field corresponding to $(*)$, i.e., $X=f \partial / \partial x+g \partial / \partial y$. As usual, let $\operatorname{div} X$ be the divergence of $X$ (with respect to the ordinary area-element $d x d y$ ), i.e., $\operatorname{div} X=f_{x}+g_{y}$. We consider the following condition:

$$
\begin{aligned}
(X \operatorname{div} X)(x, y)=\{ & \left.f(\operatorname{div} X)_{x}+g(\operatorname{div} X)_{y}\right\}(x, y)<0 \\
& \quad \text { for any }(x, y) \in \Sigma=\{(x, y) \mid(\operatorname{div} X)(x, y)=0\} .
\end{aligned}
$$

This condition means that for each point $p$ in $\Sigma$, the tangent vector $X_{p}$ at $p$ points into the set $\{(x, y) \mid(\operatorname{div} X)(x, y)<0\}$. Therefore, if $(*)$ satisfies condition (D), then each closed integral curve for $(*)$ is contained in a connected component of $R^{2}-\Sigma$, and the function $\operatorname{div} X$ has a constant sign along each closed integral curve; hence each closed integral curve is positively or negatively asymptotically stable according as it is contained in $\{(x, y) \mid(\operatorname{div} X)(x, y)<0\}$ or in $\{(x, y) \mid(\operatorname{div} X)(x, y)>0\}$. In this way, if system $(*)$ satisfies condition (D), we can estimate the number of limit cycles of $(*)$ in terms of the topology of $\boldsymbol{R}^{2}-\Sigma$.

Now, when $(*)$ does not satisfies (D), what can we say? At this point, notice that $\operatorname{div} X$ can take a different form when we multiply $X$ by a positive function $e^{u(x, y)}$, and note that the vector field $e^{u} X$ gives exactly the same pattern as $X$. (We do not try to change the area-element on 
$\boldsymbol{R}^{2}$, for a certain reason.) Then, if we can choose a function $e^{u}$ so that the new vector field $Y=e^{u} X$ satisfies

$$
(Y \operatorname{div} Y)(x, y)<0
$$

for any $(x, y) \in \Sigma^{\prime}=\{(x, y) \mid(\operatorname{div} Y)(x, y)=0\}$, then we can say the same thing for $(*)$ as for systems satisfying condition (D).

Well, but how shall we find such a function $e^{u}$ ? Indeed, it is not easy to solve directly the partial differential inequality

$$
e^{u} X\left(\operatorname{div} e^{u} X\right)<0 \quad \text { on } \Sigma^{\prime} .
$$

Our answer is to find approximate solutions $e^{u}$ of the partial differential equation

$$
e^{u} X\left(\operatorname{div} e^{u} X\right)-\left(\operatorname{div} e^{u} X\right)^{2}=-\lambda^{2}
$$

(which is equivalent to

$$
\left.(X-\operatorname{div} X)(X u)+(X-\operatorname{div} X) \operatorname{div} X+\lambda^{2} e^{-2 u}=0\right),
$$

where $\lambda$ is a constant. Note that for the solution (or approximate solution) $e^{u}$ of $(\mathrm{E})$ with $\lambda \neq 0$, the vector field $e^{u} X$ satisfies condition $\left(\mathrm{D}^{\prime}\right)$. Hence if we find an approximate solution $e^{u}$ of $(\mathrm{E})$, then we can estimate the number of limit cycles. Furthermore, as we shall see, our knowledge of the positions of limit cycles becomes more accurate as the approximate solution of (E) becomes more correct. Thus, our problem of determining the number and the approximate positions of limit cycles reduces to the problem of finding approximate solutions of (E).

Let us explain equation (E). Analytically, the solution $e^{u}$ of (E) is the "best" function (in the sense of calculus of variations) of all functions satisfying $\left(\mathrm{D}^{\prime}\right)$. Geometrically, equation (E) means that the 1-parameter transformation group $\left\{\phi_{t}\right\}$ generated by $e^{u} X$ has the following property. Recall that $\operatorname{div}\left(e^{u} X\right)$ gives the relation between the area $\Delta_{0}(p ; U)$ of an infinitesimal neighborhood $U$ of a point $p$ in $R^{2}$ and the area $\Delta_{t}(p ; U)$ of the infinitesimal neighborhood $\phi_{t}(U)$ of the point $\phi_{t}(p)$, by the formula

$$
\Delta_{t}(p ; U)=\Delta_{0}(p ; U) \exp \int_{0}^{t}\left(\operatorname{div} e^{u} X\right)\left(\phi_{t}(p)\right) d t
$$

Equation (E) means that the function $\Delta_{t}(p ; U)$ of $t$ behaves as the function 


$$
\frac{1}{c e^{\lambda t}+(1-c) e^{-\lambda t}}
$$

where $c$ is a constant $(0 \leqslant c \leqslant 1)$ depending on the point $p$.

It should be remarked that the solution $e^{u}$ of $(\mathrm{E})$ is a "generalized" integrating factor for $X$. An integrating factor for $X$, if any, is a function $e^{u}$ satisfying $\operatorname{div}\left(e^{u} X\right)=0$. (If such a function $e^{u}$ exists, then the problem of solving $(*)$ reduces to a mere quadrature.) Note that if $e^{u}$ is an integrating factor for $X$, then $e^{u}$ satisfies (E) with $\lambda=0$. Hence the solution $e^{u}$ of $(\mathrm{E})$ can be viewed as a generalized integrating factor for $X$. Although integrating factors exist only in the exceptional case, our "integrating factors" exist for almost every $X$.

Let us now describe the contents of this paper.

In $\S 1$, we deal with a vector field $X$ defined on a closed surface $M$. On $M$ we fix an area-element $\omega$. Then we can define the divergence $\operatorname{div} X$ of $X$ and the set $\Sigma=\{p \in M \mid(\operatorname{div} X)(p)=0\}$. We begin with a geometric theorem (Theorem 1.1.1), which asserts that if $X$ satisfies

$$
(X \operatorname{div} X)(p)<0 \quad \text { for any } p \in \Sigma \text {, }
$$

then we have

the number of limit cycles $\leqslant \#\{\Sigma\}-1+$ the genus of $M$, where $\#\{\Sigma\}$ is the number of connected components of $\Sigma$.

The lower bound of the number of limit cycles is given by Proposition 1.2.1.

One of the main results of this paper is Theorem 1.1.2 which makes Theorem 1.1.1 useful. Theorem 1.1.2 asserts that if $X$ is "strongly" structurally stable, then there exists a function $e^{u}: M \rightarrow R$ such that

$$
\left(e^{u} X-\operatorname{div} e^{u} X\right)\left(\operatorname{div} e^{u} X\right)=-\lambda^{2},
$$

where $\lambda$ is a positive constant.

Practically the following (Theorem 1.2.2.) is more important. If $e^{v}$ is an approximate solution of (E), i.e., if $e^{v}$ satisfies

$$
-(\lambda+\varepsilon)^{2}<\left(e^{v} X-\operatorname{div} e^{v} X\right)\left(\operatorname{div} e^{v} X\right)<-(\lambda-\varepsilon)^{2}
$$

with constants $\varepsilon, \lambda, 0<\varepsilon<\lambda$, then the set

$$
\left\{p \in M \mid\left(\operatorname{div} e^{v} X\right)^{2}(p) \geqslant(\lambda-\varepsilon)^{2}\right\}
$$


contains all limit cycles. Moreover, this set approaches the limit cycles (and singular points and separatrices) as $\varepsilon \rightarrow 0$.

By this theorem we can get the approximate positions of limit cycles as accurately as we want. Let us roughly explain this theorem. If a vector field $e^{u} X$ satisfies (E), then the set $\left\{p \in M \mid\left(\operatorname{div} e^{u} X\right)^{2}=\lambda^{2}\right\}$ is an invariant set and contains all the closed integral curves (which are necessarily limit cycles). Hence if a function $e^{v}$ approximates the function $e^{u}$, then the set $\left\{p \in M \mid\left(\operatorname{div} e^{v} X\right)^{2}=\lambda^{2}\right\}$ also approximates the set above for $e^{u} X$, and so gives the approximate positions of limit cycles.

1.3 and 1.4 are the proofs of Theorems 1.1.1 and 1.2.2 respectively. It may be noted that the formula in Lemma 1.4.2 gives a new type of relation between a curvilinear integral and a surface integral. We end this section by giving an example satisfying condition (D).

In $\S 2$ (which can be read independently of the rest of this paper), we discuss the applications of our method to Liénard's equations

$$
\left\{\begin{array}{l}
\dot{x}=y-f(x) \\
\dot{y}=-x, \quad f(-x)=-f(x) .
\end{array}\right.
$$

In order to solve equation $(\mathrm{E})$, with $X=(y-f) \partial / \partial x-x \partial / \partial y$, we use a perturbation technique (except 2.5).

We begin with the modifications of Theorem 1.1.1 adapted for Liénard's equations, and in 2.2 we give the approximate (or formal) solution of (E). Using this approximate solution we obtain the classical result (cf. Lefschetz $[9$, p. 320]) that the generating circles of limit cycles are given by the zeros of $\int_{0}^{2 \pi} \cos \theta f(r \cos \theta) d \theta$.

In 2.3 we study the case $f(x)=\mu \sin x$ and prove the result (due to Hochstadt, Stephan [6], and D'heedene [3]) that the system

$$
\left\{\begin{array}{l}
\dot{x}=y-\mu \sin x \\
\dot{y}=-x \quad(\mu: \text { nonzero constant })
\end{array}\right.
$$

has an infinite number of limit cycles.

In order to explain 2.4 and 2.5, we have to note that equation (E) may reduce to the equation

$$
\operatorname{div}\left(e^{u} X\right)= \pm \lambda \text {. }
$$

It is geometrically obvious that if a vector field $X$ on $R^{2}$ has a unique 
limit cycle with nontrivial characteristic exponent and has a unique singular point, say $(0,0)$, then there is a function $e^{u}: \boldsymbol{R}^{2}-\{(0,0)\} \rightarrow \boldsymbol{R}$ such that the divergence $\operatorname{div}\left(e^{u} X\right)$ has a constant sign. (Indeed, as mentioned in 2.1, we can make $\operatorname{div}\left(e^{u} X\right)=$ constant.) Recall that instead of multiplying $X$ by $e^{u}$, if we multiply the area-element $d x d y$ by $e^{u}$, then the divergence $\operatorname{div}_{\omega} X$ of $X$ with respect to the area-element $\omega=e^{u} d x d y$ is given by $e^{-u}\left(\operatorname{div} e^{u} X\right)=X u+\operatorname{div} X$. Therefore, the equation $\operatorname{div}_{\omega} X=$ constant is linear (in $u$ ), while the equation $\operatorname{div}\left(e^{u} X\right)=$ constant is nonlinear. Thus in order to study a Liénard's equation with a unique limit cycle (or without limit cycle), we had better deal with the equation $\operatorname{div}_{\omega} X=$ constant. Under this view, the van der Pol equation is discussed in 2.4, and the Liénard's theorem is proved in 2.5.

$\S 3$ is devoted to the proof of Theorem 1.1.2. Here it may be appropriate to insert the geometric idea for the proof of this theorem. In order to simplify the explanation, let us deal with a nonsingular vector field $X$ on $T^{2}=R^{2} / Z^{2}$, and suppose that $X$ has a global cross-section and that there are only two closed integral curves with nontrivial characteristic exponent. We want to give a function $e^{u}$ such that $e^{u} X$ satisfies (E). Let $\mathrm{A}, \mathrm{B}$ be the two closed annular domains bounded by the two limit cycles. Let $\alpha, \beta$ be the areas of A, B respectively. (Clearly $\alpha+\beta=1$.) Then we introduce a $C^{2}$ vector field $Y$ on $T^{2}$ as follows:

$$
Y=f(x) \frac{\partial}{\partial x}+\frac{\partial}{\partial y}
$$

where

$$
f(x)=\left\{\begin{array}{l}
\frac{\alpha \lambda}{\pi} \sin \frac{\pi}{\alpha} x \quad \text { for } x \in[0, \alpha], \\
-\frac{\beta \lambda}{\pi} \sin \frac{\pi}{\beta}(x-\alpha) \quad \text { for } x \in[\alpha, 1] .
\end{array}\right.
$$

Note that $Y$ has just two limit cycles, $x=0, x=\alpha$, and the areas of the two annular domains bounded by these limit cycles are $\alpha, \beta$ respectively. Furthermore it is easily verified that

$$
(Y-\operatorname{div} Y) \operatorname{div} Y=-\lambda^{2} .
$$

In order to get a function $e^{u}$ such that $e^{u} X$ satisfies (E), it suffices to construct a homeomorphism $h: T^{2} \rightarrow T^{2}$ (diffeomorphism almost everywhere) such that $h$ is area-preserving and such that each integral curve for $X$ 
is mapped by $h$ onto an integral curve for $Y$ (In fact, define $e^{u}$ by the formula $h_{*}\left(e^{u} X\right)=Y$ ). We construct such a homeomorphism $h$ as follows. Let $\gamma$ be a noncompact integral curve for $X$. There is a unique point $p$ on $\gamma$ such that for an infinitesimal neighborhood $U$ of $p$, the area of the (infinitesimal) domain $\bigcup_{t \geqslant 0} \phi_{t}(U)$ is equal to the area of $\bigcup_{t \leqslant 0} \phi_{t}(U)$, where $\left\{\phi_{t}\right\}$ is the 1-parameter transformation group generated by $X$. Such points $p$, when $\gamma$ runs over all noncompact integral curves, constitute two circles $S_{A} \subset A, S_{B} \subset B$. Since $h$ must be area-preserving and orbits-preserving, the circles $S_{A}, S_{B}$ must be mapped by $h$ to the circles $x=\alpha / 2, x=$ $\alpha+\beta / 2$ respectively. If the image of one point in $S_{A}$ is once determined, then the images of other points in $S_{A}$ are uniquely determined again by the requirement that $h$ is area-preserving and orbits-preserving. Similarly, the images of other points in $T^{2}$ are also determined. In this way the map $h$ is constructed.

Let us return to the contents of $\S 3$. In 3.1 we consider some geometric conditions for a vector field. Lemma 3.1.1 gives a sufficient condition in order that a pathpolygon is an $\alpha$ or $\omega$-limit pathpolygon. In 3.2 we introduce the notion of an elementary vector field and investigate its basic properties. The vector field $e^{u} X$ satisfying the condition (E) above is, by definition, an elementary vector field. A typical example of elementary vector fields is the vector field $Y$ on $T^{2}$ mentioned above. The geometric conditions in 3.1 are, roughly speaking, the geometric characterization of elementary vector fields. The precise formulation is Theorem 3.3.1, which is more general than Theorem 1.1.2. A variant of Theorem 3.3.1 is Proposition 3.3.2, to whose proof 3.4-3.7 are devoted. Lastly, in 3.8 the proof of Theorem 1.1.2 is completed.

We mention Cherkas [1] for the recent review concerning limit cycles. We also mention Bellman [13] for the various methods to solve equation (E).

Finally we wish to refer to [12]. In the analysis of vector fields the divergence is one of the basic notions. The corresponding notion of differential forms is the exterior derivative. The paper [12] deals with the geometric properties of integral manifolds defined by a completely integrable 1-form by means of the exterior derivative.

The author wishes to express his appreciation to Prof. Shiraiwa who made a number of useful comments on the manuscript. 


\section{§1. The number and the approximate positions of limit cycles}

Let $M$ be a connected, orientable, closed (i.e., compact and withoutboundary) 2-dimensional manifold of class $C^{3}$, and let $\omega$ be an area-element (i.e., a nonsingular 2 -form) of class $C^{2}$ on it. We fix $\omega$.

1.1. An upper bound of the number of limit cycles of a vector field with condition (D)

Let $X$ be a vector field of class $C^{2}$ on $M$. Denote by $\Sigma$ the set of zeros of $\operatorname{div} X$, that is,

$$
\Sigma=\{p \in M \mid(\operatorname{div} X)(p)=0\} .
$$

We consider the following condition concerning $X$ :

$$
(X \operatorname{div} X)(p)<0 \quad \text { for any } p \in \Sigma \text {. }
$$

Note that condition (D) implies that $\Sigma$ is a closed 1-dimensional submanifold of class $C^{1}$ in $M$ (see 1.3) and hence $\Sigma$ is a union of a finite number of circles.

We begin with the following simple fact. It shows the importance of condition (D) and is our starting point for discussing the qualitative property of $X$.

TheOREM 1.1.1. Suppose that $X$ satisfies condition (D). Then the number of limit cycles is equal to or less than $\sharp\{\Sigma\}+g-1$, where $\sharp\{\Sigma\}$ denotes the number of connected components of $\Sigma$, and $g$ is the genus of the surface $M$. Especially, in the case where $M$ is a sphere (resp. torus), the equality holds if $X$ has, as singular points, only sources and sinks (resp. if $X$ is nonsingular).

This theorem will be proved in 1.3 .

Unfortunately, vector fields satisfying condition (D) do not make a dense subset in the set of all $C^{2}$ vector fields on $M$, although they make an open subset. In order to overcome the difficulty, we try to change the velocity of $X$ by multiplying a positive function $e^{u}$ so that the new vector field $e^{u} X$ (having obviously the same qualitative property as $X$ ) satisfies condition (D). The following theorem tells us not only the possibility but also how to seek out such a function $e^{u}$. Recall that a vector field $X$ on $M$ is said to be structurally stable if a $C^{1}$-small perturbation of $X$ does not change the qualitative property of $X$. (For the precise definition, see [10].) 
Theorem 1.1.2. Let $X$ be a vector field of class $C^{2}$ on $M$. Assume that $X$ is structurally stable, and that $(\operatorname{div} X)(p) \neq 0$ for any singular point $p$ of $X$. Then there exists uniquely a continuous function $u: M \rightarrow R$ and uniquely a positive constant $\lambda$ satisfying the following conditions:

(i) The function $u$ is of class $C^{2}$ with respect to $X$, that is, the derivatives $X u, X^{2} u$ exist, and are continuous on $M$.

(ii) The function $u$ is a solution of differential equation

$$
(X-\operatorname{div} X)(X u)+(X-\operatorname{div} X) \operatorname{div} X+\lambda^{2} e^{-2 u}=0 .
$$

(iii) The integral of $u$ on $M$ is equal to zero, that is,

$$
\int_{M} u \omega=0
$$

We shall prove this theorem in $\S 3$.

Remark. Let $X, u, \lambda$ be as above. Then, using an elementary formula $\operatorname{div} e^{u} X=e^{u}(X u+\operatorname{div} X)$, we see that the vector field $Y=e^{u} X$ satisfies the identity

$$
(Y-\operatorname{div} Y) \operatorname{div} Y=-\lambda^{2}
$$

and hence especially condition (D). (Strictly speaking, although the assumption concerning the smoothness of $Y$ is not satisfied, it makes no matter.)

1.2. A lower bound of the number and the approximate position of limit cycles

Proposition 1.2.1. Suppose that a $C^{2}$ vector field $X$ satisfies condition (D). If $X$ is structurally stable, then the number of limit cycles is equal to or greater than the number of the connected components of $M-\Sigma$ which contain neither sources nor sinks.

This is an immediate consequence of the Poincaré-Bendixson theorem, the characterization theorem of Peixoto [10], and the plus or minus invariance of each connected component of $M-\Sigma$ (see 1.3). The following theorem will be proved in 1.4 .

Theorem 1.2.2. Let $X$ be a $C^{2}$ vector field on $M$, and let $\varepsilon, \lambda$ be two numbers such that $0<\varepsilon<\lambda$. Assume that there exists a $C^{2}$ function $v: M$ $\rightarrow \boldsymbol{R}$ satisfying the inequalities 
$-(\lambda+\varepsilon)^{2} e^{-2 v}<(X-\operatorname{div} X) X v+(X-\operatorname{div} X) \operatorname{div} X<-(\lambda-\varepsilon)^{2} e^{-2 v}$.

Then the sets

$$
\begin{aligned}
M_{\lambda-\varepsilon} & =\left\{p \in M \mid e^{v}(X v+\operatorname{div} X)(p) \geqslant \lambda-\varepsilon\right\}, \\
M_{-(\lambda-\varepsilon)} & =\left\{p \in M \mid e^{v}(X v+\operatorname{div} X)(p) \leqslant-(\lambda-\varepsilon)\right\}
\end{aligned}
$$

have the following properties:

(i) The sets $M_{\lambda-8}, M_{-(2-s)}$ are 2-dimensional, compact, $C^{1}$ submanifolds (with boundary) of $M$, and are invariant in the negative direction of $X$, in the positive direction of $X$ respectively.

(ii) Every closed trajectory $\gamma$ is contained in $M_{\lambda-s} \cup M_{-(\lambda-s)}$, and is an $\alpha$ or $\omega$-limit cycle according as $\gamma$ is contained in $M_{\lambda-\varepsilon}$ or in $M_{-(\lambda-\varepsilon)}$.

(iii) The respective areas of the sets $M_{\lambda-s}, M_{-(\lambda-6)}$ are less than

$$
\frac{2}{\pi} \frac{(1+\mu)}{(1-\mu)^{2}} \cdot \arctan \left(\mu^{(1-\mu) / 2(1+\mu)}\right) \cdot \int_{M} \omega, \quad \text { where } \mu=\frac{\varepsilon}{\lambda} .
$$

Remark 1. Let $X, v$ be as above. Then the vector field $Y=e^{v} X$ satisfies condition (D). In fact, it is an immediate consequence of the inequality $(Y-\operatorname{div} Y) \operatorname{div} Y<-(\lambda-\varepsilon)^{2}$ which is equivalent to the righthand side of the above inequalities for $v$.

Remark 2. Assertion (iii) implies that if $X$ is nonsingular on $M_{\lambda-s}$ $\cup M_{-(\lambda-\varepsilon)}$, then the sets $M_{\lambda-\varepsilon}$ and $M_{-(\lambda-\varepsilon)}$ approach limit cycles as $\varepsilon \rightarrow 0$.

\subsection{Proof of Theorem 1.1.1}

Let $X$ satisfy condition (D). Since $X(\operatorname{div} X)=0$ at singular points of $X$, the set $\Sigma$ contains no singular point. This fact and condition (D) imply that any point in $\Sigma$ is not a critical point of the $C^{1}$ function $\operatorname{div} X$. By the implicit function theorem we see that $\Sigma$ is a closed 1-dimensional submanifold of class $C^{1}$ in $M$, and that $\{p \in M \mid(\operatorname{div} X)(p) \leqslant 0\},\{p \in M \mid(\operatorname{div} X)(p)$ $\geqslant 0\}$ are compact 2-dimensional submanifolds of $M$ whose boundaries coincide with $\Sigma$. Furthermore, condition (D) implies that for any point $p$ of $\Sigma$, the tangent vector of $X$ at $p$ points toward the interior of $\{p \in M \mid \operatorname{div} X$ $\leqslant 0$. From this it follows that no limit cycle can traverse $\Sigma$, and hence that each limit cycle is contained in a connected component of $\{p \in$ $M \mid(\operatorname{div} X)(p) \leqslant 0\}$ or of $\{p \in M \mid(\operatorname{div} X)(p) \geqslant 0\}$. We must therefore estimate the number of limit cycles contained in each connected component.

Let $F$ be a connected component of $\{p \in M \mid(\operatorname{div} X)(p) \leqslant 0\}$. Note that 
$F$ is a connected, compact surface with boundary. Let $\sharp\{\partial F\}$ denote the number of connected components of the boundary $\partial F$. Let $g(F)$ be the genus of the closed surface obtained by attaching $\#\{\partial F\}$ closed disks to $F$ (i.e. by identifying each boundary of the disks with each connected component of $\partial F$ ).

Lemma 1.3.1. The number of limit cycles in $F$ is equal to or less than $g(F)+\#\{\partial F\}-1$.

Proof. For simplicity of notation, we put $g=g(F), h=\sharp\{\partial F\}$. Let $H_{1}\left(F ; Z_{2}\right)$ be the 1 st real homology group of $F$ with coefficients $Z / 2 Z$. It is easy to see that the dimension of $H_{1}\left(F ; Z_{2}\right)$ is equal to $2 g+h-1$. Let $I: H_{1}\left(F ; Z_{2}\right) \times H_{1}\left(F ; Z_{2}\right) \rightarrow Z_{2}$ be the bilinear map defined by intersection. We note that the rank of $I$ is equal to $2 g$. Suppose now that there were, in $F, g+h$ limit cycles $C_{1}, C_{2}, \cdots, C_{g+h}\left(C_{i} \cap C_{j}=\phi\right.$ if $\left.i \neq j\right)$. Using the facts that the integral of $\operatorname{div} X$ on an invariant set is zero (cf. [7, p. 281]) and that $(\operatorname{div} X)(p)<0$ for any interior point $p$ of $F$, we see that any $C_{i_{1}}, C_{i_{2}}, \cdots, C_{i_{j}}$ can not bound a 2-dimensional submanifold of $F$. Hence the homology classes $\left[C_{1}\right], \cdots,\left[C_{g+h}\right]$ in $H_{1}\left(F ; Z_{2}\right)$ are linearly independent. On the other hand, it is clear that $I\left(\left[C_{i}\right],\left[C_{j}\right]\right)=0$ for any $i, j$. From these two facts we see that the rank of $I$ is equal to or less than $2 g-2$. This contradiction proves our lemma.

From this lemma it follows immediately that the number of limit cycles in $\{p \in M \mid(\operatorname{div} X)(p) \leqslant 0\}$ is equal to or less than

$$
\sum_{F}(g(F)+\#\{\partial F\}-1),
$$

where $F$ runs over all the connected components of $\{p \in M \mid(\operatorname{div} X)(p) \leqslant 0\}$. To arrange the expression, we recall that the Euler characteristic of a closed surface $S$ is equal to $2(1-$ genus of $S)$. Then we have, for each connected component $F$,

$$
\sharp\{\partial F\}+\sum_{p \in F} \operatorname{index}(p)=2(1-g(F)),
$$

where $p$ runs over the set of singular points in $F$, and index $(p)$ denotes the index of $X$ at $p$. Using this relation and the fact that

$$
\sum_{F} \sharp\{\partial F\}=\sharp\{\Sigma\},
$$

we conclude that the number of limit cycles in $\{p \in M \mid(\operatorname{div} X)(p) \leqslant 0\}$ is equal to or less than 


$$
\frac{1}{2}\left\{\#\{\Sigma\}-\sum_{\operatorname{div}} \underset{X(p) \leqslant 0}{ } \text { index }(p)\right\},
$$

where $p$ runs over the set of singular points in $\{p \in M \mid(\operatorname{div} X)(p) \leqslant 0\}$ : Clearly, we have the similar inequality for the number of limit cycles in $\{p \in M \mid(\operatorname{div} X)(p) \geqslant 0\}$. Consequently, the number of limit cycles in $M$ is equal to or less than

$$
\sharp\{\Sigma\}-\frac{1}{2} \sum_{p \in M} \operatorname{index}(p)
$$

i.e.,

$$
\sharp\{\Sigma\}+g-1 \text {. }
$$

To prove the latter part of our theorem, let us consider the case where $M$ is a torus and $X$ is nonsingular. Then we can see easily that each connected component of $\{p \in M \mid(\operatorname{div} X)(p) \leqslant 0\}$ or $\{p \in M \mid(\operatorname{div} X)(p)$ $\geqslant 0\}$ is homeomorphic to a closed annulus and contains at least one limit cycle. From this it follows that the number of connected components of $\{p \in M \mid(\operatorname{div} X)(p) \leqslant 0\},\{p \in M \mid(\operatorname{div} X)(p) \geqslant 0\}$ is equal to $\sharp\{\Sigma\}$ and hence the number of limit cycles is equal to or greater than $\sharp\{\Sigma\}$. From this and the former part of our theorem, we conclude that the number of limit cycles coincides with $\sharp\{\Sigma\}$, as desired. As for the case where $M$ is a sphere, we can prove similarly that the number of limit cycles is equal to $\sharp\{\Sigma\}-1$. (Our assumption on singular points implies that the index of each singular point is 1 . Using the Poincaré-Hopf theorem we see that $M-\Sigma$ is a union of two open disks and $\sharp\{\Sigma\}-1$ open annuluses.)

\subsection{Proof of Theorem 1.2.2}

Let the notation be as in Theorem 1.2.2. Introduce a vector field $Y=e^{v} X$. Recall the elementary formula $\operatorname{div} Y=e^{v}(X v+\operatorname{div} X)$. Then the assumption concerning $v$ can be written

$$
-(\lambda+\varepsilon)^{2}<(Y-\operatorname{div} Y) \operatorname{div} Y<-(\lambda-\varepsilon)^{2} .
$$

The sets $M_{\lambda-\varepsilon}, M_{-(\lambda-\varepsilon)}$ can be written

$$
\begin{aligned}
M_{\lambda-\varepsilon} & =\{p \in M \mid(\operatorname{div} Y)(p) \geqslant \lambda-\varepsilon\}, \\
M_{-(\lambda-\varepsilon)} & =\{p \in M \mid(\operatorname{div} Y)(p) \leqslant-(\lambda-\varepsilon)\} .
\end{aligned}
$$

and hence the boundaries of $M_{\lambda-6}, M_{-(\lambda-\varepsilon)}$ coincide with $\{p \in M \mid(\operatorname{div} Y)(p)$ $=\lambda-\varepsilon\},\{p \in M \mid(\operatorname{div} Y)(p)=-(\lambda-\varepsilon)\}$ respectively. 
To prove (i), let $p$ be a point of the boundary of $M_{\lambda-6}$. From the inequality $(Y-\operatorname{div} Y) \operatorname{div} Y<-(\lambda-\varepsilon)^{2}$, it follows that $(Y \operatorname{div} Y)(p)<0$, and hence that the tangent vector $Y_{p}$ points toward the exterior of $M_{\lambda-\varepsilon}$. It is clear that the boundary of $M_{\lambda-\varepsilon}$ is a 1-dimensional, closed manifold of class $C^{1}$, because the $C^{1}$ function $\operatorname{div} Y: M \rightarrow R$ has no critical point on the boundary of $M_{\lambda-6}$. Consequently, $M_{\lambda-\sigma}$ is a 2-dimensional compact submanifold of class $C^{1}$ in $M$ and is invariant in the negative direction of $Y$ and hence of $X$. Similarly, the assertion for $M_{-(\lambda-s)}$ can be verified.

To prove (ii) let $\gamma(s)$ be a closed trajectory for $Y$. By the mean value theorem we see that there is a number $s_{0}$ such that $d /\left.d s\right|_{s=s_{0}}(\operatorname{div} Y)(\gamma(s))$ $=0$, i.e. $(Y \operatorname{div} Y)\left(\gamma\left(s_{0}\right)\right)=0$. Hence $\left\{(\operatorname{div} Y)\left(\gamma\left(s_{0}\right)\right)\right\}^{2}>(\lambda-\varepsilon)^{2}$. This means that $\gamma\left(s_{0}\right) \in M_{\lambda-\varepsilon} \cup M_{-(\lambda-\varepsilon)}$. Part (i) shows that $\gamma(s) \in M_{\lambda-\varepsilon} \cup M_{-(\lambda-\varepsilon)}$ for all $s$. The latter part of (ii) is obvious.

To prove (iii), we begin with a comparison theorem.

LEMMA 1.4.1. Let $a: R \rightarrow \boldsymbol{R}$ be a continuous function such that

$$
(\lambda-\varepsilon)^{2}<-a(s)<(\lambda+\varepsilon)^{2}
$$

for all $s \in R$, where $\lambda, \varepsilon$ are constants satisfying $\lambda>\varepsilon>0$. Let $h=h(s)$ be the solution of a differential equation

$$
\frac{d^{2} h}{d s^{2}}=-a(s) h
$$

with initial condition $h(0)=1,(d h / d s)(0)=0$. Then $h$ satisfies the following inequalities:

$$
\begin{gathered}
\cosh (\lambda-\varepsilon) s \leqslant h(s) \leqslant \cosh (\lambda+\varepsilon) s \quad \text { for all } s \in \boldsymbol{R}, \\
\frac{h^{\prime}(s)}{h(s)} \leqslant(\lambda+\varepsilon) \tanh (\lambda+\varepsilon) s \quad \text { for } \text { all } s \geqslant 0 .
\end{gathered}
$$

$\left(\right.$ Here $h^{\prime}(s)=d h / d s$, as usual. $)$

Proof. Recall that

$$
\cosh (\lambda \pm \varepsilon) s=\frac{1}{2}\left(e^{(\lambda \pm \sigma) s}+e^{-(\lambda \pm \varepsilon) s}\right)
$$

and that the functions $g(s)=\cosh (\lambda \pm \varepsilon) s$ satisfy the differential equations

$$
\frac{d^{2} g}{d s^{2}}=(\lambda \pm \varepsilon)^{2} g
$$

with initial condition $g(0)=1, g^{\prime}(0)=0$. Now, consider two functions 


$$
\eta_{ \pm}(s)=\cosh (\lambda \pm \varepsilon) s-h(s) .
$$

To prove $\eta_{+}(s) \geqslant 0, \eta_{-}(s) \leqslant 0$, we observe that $\eta_{ \pm}(0)=0, \eta_{ \pm}^{\prime}(0)=0$, and that

$$
\frac{d^{2} \eta_{+}(s)}{d s^{2}}>-a(s) \eta_{+}(s), \quad \frac{d^{2} \eta_{-}(s)}{d s^{2}}<-a(s) \eta_{-}(s) \text {. }
$$

Since $-a(s)>0$, we conclude that $\eta_{+}(s)>0$ for $s \neq 0$, and $\eta_{-}(s)<0$ for $s \neq 0$. Consequently, we obtain

$$
\eta_{+}(s) \geqslant 0, \quad \eta_{-}(s) \leqslant 0
$$

for all $s$, which show that $h$ satisfies the former inequalities in our lemma. To prove the latter inequality, note that $G(s)=(\lambda+\varepsilon) \tanh (\lambda+\varepsilon) s$ satisfies the differential equation

$$
\frac{d G}{d s}=(\lambda+\varepsilon)^{2}-G^{2}
$$

with initial condition $G(0)=0$. Using the inequality $-a(s)<(\lambda+\varepsilon)^{2}$, we observe that $g(s)=h^{\prime}(s) / h(s)$ satisfies the differential inequality

$$
\frac{d g}{d s}<(\lambda+\varepsilon)^{2}-g^{2}
$$

with initial condition $g(0)=0$. By the usual argument we conclude that $g(s)<G(s)$ for $s>0$, and hence that $g(s) \leqslant G(s)$ for all $s \geqslant 0$. This completes the proof of the lemma.

We prepare another lemma. Recall that $Y=e^{v} X$. Let $\left\{\psi_{s}\right\}$ be the 1-parameter transformation group generated by $Y$. For a 2-dimensional compact submanifold $N$ of $M$, we introduce the following notations. Denote by $\partial N$ the boundary of $N$, and by area $(N)$ the area of $N$, i.e.,

$$
\operatorname{area}(N)=\int_{N} \omega
$$

We denote by $\psi_{s}(N)$ the set $\left\{\psi_{s}(p) \mid p \in N\right\}$. Note that the 2-form $\left.\omega\right|_{N}$ on $N$ defines naturally an orientation of $N$, and the orientation of $N$ defines an orientation of $\partial N$ in the canonical way.

Lemma 1.4.2. Let $N$ be a 2-dimensional compact submanifold of $M$. Assume that there exist two positive constants $\mu, \nu$ such that $(\operatorname{div} Y)\left(\psi_{s}(x)\right)$ $<-\nu$ for all $s>\mu$ and all $x \in N$. Then we have 


$$
\operatorname{area}\left(\psi_{s}(N)\right)=-\int_{x \in \partial N}\left\{\int_{s}^{\infty}\left(\exp \int_{0}^{\sigma}(\operatorname{div} Y)\left(\psi_{\sigma}(x)\right) d \sigma\right) d \sigma\right\} \iota_{Y} \omega
$$

for any $s \in R$, where $\iota_{Y}$ is the interior product of $Y$ and $\omega$, i.e., the 1-form defined by $\left(\iota_{Y} \omega\right)(\xi)=\omega(Y, \xi)$.

Proof. Consider two functions

$$
\begin{aligned}
& f(s)=\operatorname{area}\left(\psi_{s}(N)\right) \\
& g(s)=-\int_{x \in \partial N}\left\{\int_{s}^{\infty}\left(\exp \int_{0}^{\sigma}(\operatorname{div} Y)\left(\psi_{\sigma}(x)\right) d \sigma\right) d \sigma\right\} \iota_{Y} \omega .
\end{aligned}
$$

Note that for a fixed $s$, the integral

$$
\int_{s}^{\infty}\left(\exp \int_{0}^{\sigma}(\operatorname{div} Y)\left(\psi_{\sigma}(x)\right) d \sigma\right) d \sigma
$$

converges uniformly for $x$ in $\partial N$, and hence is continuous on $\partial N$, because

$$
\exp \int_{0}^{\sigma}(\operatorname{div} Y)\left(\psi_{\sigma}(x)\right) d \sigma \leqslant \text { constant } \cdot e^{-\nu \sigma}
$$

for any $\sigma \geqslant 0$ and any $x \in \partial N$. It is obvious that $\lim _{s \rightarrow \infty} f(s)=0=\lim _{s \rightarrow \infty} g(s)$. We contend that $f^{\prime}(s)=g^{\prime}(s)$. This will prove that $f(s)=g(s)$. Using elementary properties of Lie differentiation $\mathscr{L}_{Y}$, we observe that

$$
\begin{aligned}
f^{\prime}(s) & =\frac{d}{d s} \int_{\psi_{s}(N)} \omega=\frac{d}{d s} \int_{N} \psi_{s}^{*} \omega=\int_{N} \mathscr{L}_{Y}\left(\psi_{s}^{*} \omega\right) \\
& =\int_{N} \psi_{s}^{*}\left(\mathscr{L}_{Y} \omega\right)=\int_{N} \psi_{s}^{*}((\operatorname{div} Y) \omega)=\int_{\psi_{s}(N)}(\operatorname{div} Y) \omega .
\end{aligned}
$$

On the other hand, using the fact that the pull-back $\psi_{s}^{*} \omega$ of $\omega$ by $\psi_{s}$ is given by

$$
\left\{\exp \int_{0}^{s}(\operatorname{div} Y)\left(\psi_{\sigma}(x)\right) d \sigma\right\} \omega
$$

and recalling the formula $\mathscr{L}_{Y} \omega=d\left(\iota_{Y} \omega\right)$ (cf. [7, p. 282]), we observe that

$$
\begin{aligned}
g^{\prime}(s) & =\int_{\partial N}\left\{\exp \int_{0}^{s}(\operatorname{div} Y)\left(\psi_{\sigma}(x)\right) d \sigma\right\} \iota_{Y} \omega=\int_{\partial N} \iota_{Y}\left(\psi_{s}^{*} \omega\right) \\
& =\int_{\partial\left(\psi_{s}(N)\right)} \iota_{Y} \omega=\int_{\psi_{s}(N)}(\operatorname{div} Y) \omega .
\end{aligned}
$$

Consequently, we obtain $f^{\prime}(s)=g^{\prime}(s)$, as desired. This proves our lemma.

Now, we return to the proof of Theorem 1.2.2. Consider a 2-dimensional compact submanifold 


$$
N=\{p \in M \mid(\operatorname{div} Y)(p) \leqslant 0\} .
$$

Let $\sigma$ be a positive number defined by the formula

$$
\lambda-\varepsilon=(\lambda+\varepsilon) \tanh (\lambda+\varepsilon) \sigma .
$$

We contend that

$$
\begin{gathered}
\operatorname{area}\left(\psi_{\sigma}(N)\right)=-\int_{\partial N}\left\{\int_{\sigma}^{\infty}\left(\exp \int_{0}^{s}(\operatorname{div} Y)\left(\psi_{s}(x)\right) d s\right) d s\right\} \iota_{Y} \omega, \\
M_{-(\lambda-s)} \subset \psi_{\sigma}(N) .
\end{gathered}
$$

Formula (1) is a direct consequence of Lemma 1.4.2 and the assumption $(Y-\operatorname{div} Y) \operatorname{div} Y<-(\lambda-\varepsilon)^{2}$. To prove (2), it suffices to prove that

$$
\psi_{\sigma}(p) \notin M_{-(2-\sigma)}-\partial M_{-(2-\varepsilon)}
$$

for any $p$ in $\partial N$, because $M_{-(\lambda-s)}, N$ are invariant by $\psi_{s}(s \geqslant 0)$, and $M_{-(\lambda-s)}$ $\subset N$. To prove this, fix a point $p$ in $\partial N$, and consider a function

$$
h(s)=\exp \left\{-\int_{0}^{s}(\operatorname{div} Y)\left(\psi_{s}(p)\right) d s\right\} .
$$

Then $h(s)$ satisfies $h(0)=1, h^{\prime}(0)=0$. Furthermore,

$$
\begin{aligned}
h^{\prime}(s) & =-(\operatorname{div} Y)\left(\psi_{s}(p)\right) h(s), \\
h^{\prime \prime}(s) & =-\left(Y \operatorname{div} Y-(\operatorname{div} Y)^{2}\right)\left(\psi_{s}(p)\right) h(s) .
\end{aligned}
$$

Applying Lemma 1.4.1 to $h(s)$ and $a(s)=\left(Y \operatorname{div} Y-(\operatorname{div} Y)^{2}\right)\left(\psi_{s}(p)\right)$, we obtain

$$
\cosh (\lambda-\varepsilon) s \leqslant \exp \left\{-\int_{0}^{s}(\operatorname{div} Y)\left(\psi_{s}(p)\right) d s\right\} \leqslant \cosh (\lambda+\varepsilon) s
$$

for all $s \in R$, and

$$
-(\operatorname{div} Y)\left(\psi_{s}(p)\right) \leqslant(\lambda+\varepsilon) \tanh (\lambda+\varepsilon) s
$$

for all $s \geqslant 0$. From (4) and our definition of $\sigma$, it follows that

$$
(\operatorname{div} Y)\left(\psi_{\sigma}(p)\right) \geqslant-(\lambda-\varepsilon),
$$

and hence that

$$
\psi_{\sigma}(p) \notin\{q \in M \mid(\operatorname{div} Y)(q)<-(\lambda-\varepsilon)\} .
$$

Consequently, we see that $M_{-(\lambda-s)} \subset \psi_{\sigma}(N)$, as desired.

Next, we contend that 


$$
-\frac{2}{\lambda+\varepsilon} \arctan \left(e^{-(\lambda+\varepsilon) \sigma}\right) \int_{\partial N} \iota_{Y} \omega \leqslant \operatorname{area}\left(\psi_{\sigma}(N)\right)
$$

$$
\operatorname{area}\left(\psi_{\sigma}(N)\right) \leqslant-\frac{2}{\lambda-\varepsilon} \arctan \left(e^{-(\lambda-\varepsilon) \sigma}\right) \int_{\partial N} \iota_{Y} \omega
$$

$$
-\frac{\pi}{2(\lambda+\varepsilon)} \int_{\partial N} \iota_{Y} \omega \leqslant \operatorname{area}(N) \leqslant-\frac{\pi}{2(\lambda-\varepsilon)} \int_{\partial N}{ }^{\iota_{Y}} \omega .
$$

To prove these inequalities, we use the formulas

$$
\int_{s}^{\infty} \frac{1}{\cosh (\lambda \pm \varepsilon) s} d s=\frac{2}{\lambda \pm \varepsilon} \arctan \left(e^{-(\lambda \pm \varepsilon) s}\right)
$$

(Note that $\arctan 1 / x=\pi / 2-\arctan x$.) Furthermore, we note that

$$
\int_{\partial N} \iota_{Y} \omega<0
$$

because of the definition of the orientation of $\partial N$ and because of the positive invariance of $N$ with respect to $Y$. Then, from (1), (3), we obtain (5), (5'), and (6). Moreover, these inequalities yield

$$
\frac{\operatorname{area}\left(\psi_{0}(N)\right)}{\operatorname{area}(N)} \leqslant \frac{4(\lambda+\varepsilon)}{\pi(\lambda-\varepsilon)} \arctan \left\{\left(\frac{\varepsilon}{\lambda}\right)^{(\lambda-\varepsilon) / 2(\lambda+\varepsilon)}\right\} .
$$

In fact, by definition, we see that

$$
e^{-(\lambda-\varepsilon) \sigma}=\left(\frac{\varepsilon}{\lambda}\right)^{(\lambda-\varepsilon) / 2(\lambda+\varepsilon)} .
$$

Hence, from ( $\left.5^{\prime}\right)$ and the left-hand side of (6), we get at once (7).

To prove (iii) of our theorem, we need another inequality:

$$
\operatorname{area}(N) \leqslant \frac{\lambda}{2(\lambda-\varepsilon)} \operatorname{area}(M)
$$

To prove this, we consider the set $P=\{p \in M \mid(\operatorname{div} Y)(p) \geqslant 0\}$. Then, we have

$$
\frac{\pi}{2(\lambda+\varepsilon)} \int_{\partial P} \iota_{Y} \omega \leqslant \operatorname{area}(P) \leqslant \frac{\pi}{2(\lambda-\varepsilon)} \int_{\partial P} \iota_{Y} \omega
$$

which correspond to (6). Since area $(M)=$ area $(N)+$ area $(P)$, it is obvious that

$$
\operatorname{area}(N)=\frac{1}{2} \operatorname{area}(M)+\frac{1}{2}(\operatorname{area}(N)-\operatorname{area}(P)) .
$$


Then, from the right-hand side of (6), the left-hand side of $\left(6^{\prime}\right)$, and the fact that $\partial N=-\partial P$ (as oriented manifolds), it follows that

$$
\operatorname{area}(N) \leqslant \frac{1}{2} \operatorname{area}(M)+\frac{\varepsilon \pi}{2(\lambda-\varepsilon)(\lambda+\varepsilon)} \int_{\partial P} \iota_{Y} \omega .
$$

On the other hand, by the left-hand sides of $(6),\left(6^{\prime}\right)$, we see that

$$
\frac{\pi}{\lambda+\varepsilon} \int_{\partial P} \iota_{Y} \omega \leqslant \operatorname{area}(M) .
$$

Consequently, we get

$$
\operatorname{area}(N) \leqslant \frac{\lambda}{2(\lambda-\varepsilon)} \operatorname{area}(M)
$$

Now, using (2), (7), and (8), we obtain immediately

$$
\operatorname{area}\left(M_{-(\lambda-\varepsilon)}\right) \leqslant \frac{2 \lambda(\lambda+\varepsilon)}{\pi(\lambda-\varepsilon)^{2}} \cdot \arctan \left\{\left(\frac{\varepsilon}{\lambda}\right)^{(\lambda-\varepsilon) / 2(\lambda+\varepsilon)}\right\} \cdot \operatorname{area}(M) \text {. }
$$

This is one of the desired inequalities. Similarly, we obtain the inequality for area $\left(M_{\lambda-\varepsilon}\right)$. This completes the proof of our theorem.

1.5. An example satisfying condition (D)

We shall give a simple example to which we can apply Theorem 1.1.1.

Let $T^{2}$ be the torus $\left\{(x, y) \in R^{2}\right\} / Z^{2}$, and let $\omega=d x d y$ (i.e. the ordinary area-element on $T^{2}$ ). Let $f(x, y)$ be a $C^{2}$ function on $T^{2}$, and let $\mu$ be a real number. Consider a vector field

$$
X=\mu f(x, y) \frac{\partial}{\partial x}+\frac{\partial}{\partial y}
$$

on $T^{2}$. Since $\operatorname{div} X=\mu f_{x}$ and

$$
X(\operatorname{div} X)-(\operatorname{div} X)^{2}=\mu^{2}\left(f f_{x x}-f_{x}^{2}\right)+\mu f_{x y},
$$

we have:

If $f f_{x x}-f_{x}^{2}<0$ on $T^{2}$, then for $\mu$ with sufficiently large $|\mu|$, the vector field $X$ satisfies condition (D), and hence the number of limit cycles of $X$ is equal to the number of connected components of $\Sigma=\left\{(x, y) \in T^{2} \mid f_{x}(x, y)=0\right\}$.

\section{§2. Application to Liénard's equations}

Let us consider a system of the form 


$$
\left\{\begin{array}{l}
\dot{x}=y-f(x) \\
\dot{y}=-x, \quad f(-x)=-f(x),
\end{array}\right.
$$

on an $(x, y)$-plane $\boldsymbol{R}^{2}$. In this section, as an application of our theory, we investigate the number of limit cycles of $(L)$ in some cases. We shall discuss in 2.3 the case $f(x)=\mu \sin x$, and in 2.4 the case $f(x)=\mu\left(x^{3}-x\right)$, where $\mu$ is a constant. In the previous section, we studied under the assumption that the surface on which $X$ is defined is compact. For the study of (L), we have to reformulate the assertions in $\S 1$.

2.1. Modifications of Theorem 1.1.1

Let $X$ be the vector field

$$
X=(y-f(x)) \frac{\partial}{\partial x}-x \frac{\partial}{\partial y}
$$

on $R^{2}$. We assume that $f$ is an odd function of $x$. For simplicity, we assume that $f$ is of class $C^{\infty}$. Let $\omega=e^{w(x, y)} d x d y$ be an area element of class $C^{\infty}$ on $R^{2}$. (In practice, we shall deal with the usual case $\omega=$ $d x d y$.) Denote by $\operatorname{div} X$ the divergence of $X$ with respect to $\omega$. It is an elementary fact that $\operatorname{div} X=X w-f_{x}$. Moreover, for a $C^{1}$ function $u: R^{2}$ $\rightarrow R$, we have

$$
\operatorname{div}\left(e^{u} X\right)=(X+\operatorname{div} X) e^{u}=e^{u}(X u+\operatorname{div} X) .
$$

(We consider $X+\operatorname{div} X$ as an operator.) It is obvious that $e^{u} X$ has the same qualitative property as $X$. In order to investigate the system (L) or the vector field $X$, we modify Theorem 1.1.1 as follows.

Theorem 2.1.1. Assume that there exists a $C^{2}$ function $u: R^{2} \rightarrow R$ such that the vector field $Y=e^{u} X$ satisfies the following condition:

$$
(Y \operatorname{div} Y)(x, y)<0 \quad \text { for any }(x, y) \in \Sigma,
$$

where

$$
\Sigma=\{(x, y) \mid(\operatorname{div} Y)(x, y)=0\} .
$$

Let $n(0 \leqslant n \leqslant \infty)$ be the number of compact connected components of $\Sigma$. Then the number of limit cycles of (L) is equal to $n-1$ or $n$.

Proof. If $\Sigma=\phi$, then $\operatorname{div} Y$ has a fixed sign on $R^{2}$ and hence there is no limit cycle (Criterium of Bendixson [9, p. 238]). Suppose now $\Sigma \neq \phi$. 
Note that $\Sigma$ is written as a disjoint union

$$
\Sigma=J_{1} \cup J_{2} \cup \cdots \cup J_{n} \cup L_{1} \cup \cdots \cup L_{m}
$$

where $J_{i}$ are Jordan curves, $L_{k}$ are open, complete lines, and $0 \leqslant m \leqslant \infty$. This is an immediate consequence of condition $\left(\mathrm{D}^{\prime}\right)$ and the implicit function theorem (applied to the function $\operatorname{div} Y: \boldsymbol{R}^{2} \rightarrow \boldsymbol{R}$ ). Furthermore, we observe that each trajectory $\gamma$ intersects $\Sigma$ transversely (if there is any intersection), and the intersection of $\gamma$ and each connected component of $\Sigma$ is at most one point.

Now, if $n=0$, then there is no limit cycle. Because if there were a limit cycle $C$, then by Green's theorem we should have $\int_{D}(\operatorname{div} Y) \omega=0$ where $D$ is the disk whose boundary is $C$. Hence there would be a point $p$ in $D$ such that $(\operatorname{div} Y)(p)=0$. Hence $p \in L_{i}$ for some $i$. Since $L_{i}$ is open and complete, we should conclude that $L_{i} \cap C$ consists of at least two points. This is a contradiction.

Suppose now $n \geqslant 1$. Since the only singular point of $X$ is the origin, we may assume that $J_{1}, \cdots, J_{n}$ are ordered so that the domain bounded by $J_{1}$ is a disk which contains no other $J_{j}$, and so that the domain bounded by $J_{i}$ and $J_{i+1}(1 \leqslant i \leqslant n-1)$ is an annular domain which contains no other $J_{j}$. It is obvious that the disk bounded by $J_{1}$ contains no limit cycle, because $\operatorname{div} Y$ has a fixed sign on the interior of the disk. Furthermore, we see that each of the $n-1$ annular domains contains a unique limit cycle, because each annular domain is invariant in the positive or negative direction of $X$, and $\operatorname{div} Y$ has a fixed sign on the interior of the annular domain. Hence there exist exactly $j-1$ limit cycles in the domain bounded by $J_{j}(j=1, \cdots, n)$. If $n=\infty$, our theorem is obviously proved. Suppose $n<\infty$. Let $U$ be the unbounded connected component of $R^{2}-J_{n}$. By the same argument as above, we conclude that the number of limit cycles in $U$ is at most one. This proves Theorem 2.1.1.

Considering Theorem 1.1.2, we have a more precise modification of Theorem 1.1.1.

Theorem 2.1.2. Assume that there exists a $C^{2}$ function $u: R^{2} \rightarrow R$ and $a$ positive constant $\varepsilon$ such that the vector field $Y=e^{u} X$ satisfies the inequality

$$
(Y-\operatorname{div} Y) \operatorname{div} Y<-\varepsilon^{2},
$$


which is equivalent to

$$
(X-\operatorname{div} X) X u+(X-\operatorname{div} X) \operatorname{div} X+\varepsilon^{2} e^{-2 u}<0 .
$$

Furthermore, assume that $\int_{R^{2}} \omega=\infty$. Then the number of limit cycles of $(\mathrm{L})$ is equal to the number of compact connected components of $\Sigma=$ $\{(x, y) \mid(\operatorname{div} Y)(x, y)=0\}$.

Proof. From our assumption $(Y-\operatorname{div} Y) \operatorname{div} Y<-\varepsilon^{2}$, it follows at once that the condition $\left(\mathrm{D}^{\prime}\right)$ in the preceding theorem is satisfied. We use the same notation as in the proof of the theorem. To prove our theorem, it suffices to prove that if $n<\infty$, the set $U$ (the unbounded connected component of $R^{2}-J_{n}$ ) contains at least one limit cycle. To prove this, we may assume that the vector field $X$ points outward along $J_{n}$. Denote by $C^{+}\left(J_{n}\right)$ the union of positive half trajectories starting at points in $J_{n}$. It is clear that $C^{+}\left(J_{n}\right)-J_{n}$ is an open set in $R^{2}$ and contained in $U$, and that $J_{n}$ is a connected component of the boundary $\partial C^{+}\left(J_{n}\right)$. We contend that

$$
C^{+}\left(J_{n}\right) \subsetneq J_{n} \cup U \text {. }
$$

Indeed, from the assumption $(Y-\operatorname{div} Y) \operatorname{div} Y<-\varepsilon^{2}$, we see that there is a positive constant $\varepsilon_{0}$ such that

$$
\operatorname{div} Y<-\varepsilon_{0} \text { on } C^{+}\left(J_{n}\right)-N\left(J_{n}\right),
$$

where $N\left(J_{n}\right)$ is a neighborhood of $J_{n}$. Using this inequality, we observe that the area of $C^{+}\left(J_{n}\right)$ is finite, i.e.,.

$$
\int_{C+\left(J_{n}\right)} \omega<\infty
$$

From the assumption $\int_{R^{2}} \omega=\infty$, we conclude that $C^{+}\left(J_{n}\right) \subsetneq J_{n} \cup U$, as desired. Hence $\partial C^{+}\left(J_{n}\right)-J_{n} \neq \phi$. Let $\gamma$ be a trajectory passing through a point in $\partial C^{+}\left(J_{n}\right)-J_{n}$. It is obvious that $\gamma \subset \partial C^{+}\left(J_{n}\right)-J_{n}$. If either the $\alpha$-limit set or the $\omega$-limit set of $\gamma$ is nonempty, then by the PoincaréBendixson theorem we conclude that there is a limit cycle in $U$. On the other hand, since our system $(\mathrm{L})$ is of the special form, we have the following:

LEMMA 2.1.3. There is no trajectory of $(\mathrm{L})$ whose $\alpha$ and $\omega$-limit sets both are empty. 
This lemma is an immediate consequence of the following two facts (proved by the argument similar to [5, p. 219]).

(i) If a trajectory $\gamma$ through a point $\left(x_{0}, f\left(x_{0}\right)\right)\left(x_{0} \neq 0\right)$ has not the origin $(0,0)$ as its $\alpha$-limit point or $\omega$-limit point, then $\gamma$ intersects the positive $y$-axis $\{(0, y) \mid y>0\}$ and the negative $y$-axis $\{(0, y) \mid y<0\}$.

(ii) For any $y_{+}, y_{-}\left(y_{+}>0, y_{-}<0\right)$, either the positive half trajectory starting at $\left(0, y_{+}\right)$or the negative half trajectory starting at $\left(0, y_{-}\right)$intersects the curve $\{(x, f(x)) \mid x>0\}$.

Consequently our theorem is proved.

In the study of the equation of van der Pol, the following theorem will be useful.

THEOREM 2.1.4. Assume that $f_{x}(0)<0$, and that $\int_{R^{2}} \omega=\infty$. Furthermore, assume that there exists a $C^{1}$ function $u: R^{2}-\{(0,0)\} \rightarrow R$ and $a$ positive constant $\varepsilon$ such that the vector field $Y=e^{u} X$ on $R^{2}-\{(0,0)\}$ satisfies

$$
\operatorname{div} Y<-\varepsilon \text { on } R^{2}-\{(0,0)\} \text {. }
$$

Then there exists uniquely a closed integral curve for $(\mathrm{L})$. This integral curve is asymptotically stable.

Proof. The assumption $f_{x}(0)<0$ implies that the origin $(0,0)$ is a source of $(\mathrm{L})$. Hence there is a Jordan curve $J$ about $(0,0)$ such that the vector field $X$ points outward along $J$. The same argument as in the proof of the preceding theorem shows that the unbounded connected component of $R^{2}-J$ contains a unique limit cycle. It is obvious that the limit cycle is asymptotically stable.

Remark. Assume that $f_{x}(0) \neq 0$, and assume that

$$
\int_{0}^{\tau}(\operatorname{div} X)(\gamma(t)) d t \neq 0
$$

for each nontrivial periodic solution $\gamma(t)$, with period $\tau$, of $(L)$. Let $\lambda$ be a positive real number. Then there exists a continuous function $u: R^{2}$ $\rightarrow \boldsymbol{R}$ such that

(i) the derivatives $X u, X(X u)$ exist and are continuous on $R^{2}$.

(ii) the vector field $Y=e^{u} X$ satisfies

$$
(Y-\operatorname{div} Y) \operatorname{div} Y=-\lambda^{2} .
$$

If, in addition, we assume that (L) has only one limit cycle, then we can 
take a continuous function $u^{\prime}: \boldsymbol{R}^{2}-\{(0,0)\} \rightarrow \boldsymbol{R}$ such that

(i) the derivative $X u^{\prime}$ exists on $R^{2}-\{(0,0)\}$ and is continuous.

(ii) the vector field $Y=e^{u^{\prime}} X$ satisfies

$$
(\operatorname{div} Y)^{2}=\lambda^{2} \quad \text { on } \quad R^{2}-\{(0,0)\} .
$$

The proof of these facts is similar to the one of Theorem 1.1.2.

2.2. Approximate solutions of $\left(e^{u} X-\operatorname{div}\left(e^{u} X\right)\right) \operatorname{div}\left(e^{u} X\right)=-\lambda^{2}$

From 2.1 we know that the problem of counting the number of limit cycles of $(\mathrm{L})$ is reduced to the one of solving the partial differential inequality

$$
(X-\operatorname{div} X) X u+(X-\operatorname{div} X) \operatorname{div} X+\varepsilon^{2} e^{-2 u}<0,
$$

where $\varepsilon$ is a positive constant. Furthermore, we know that if the system (L) is "structurally stable", then there is a function $u$ satisfying the partial differential equation

$$
(X-\operatorname{div} X) X u+(X-\operatorname{div} X) \operatorname{div} X+\lambda^{2} e^{-2 u}=0,
$$

where $\lambda$ is an arbitrary positive constant. (Equation (E) is equivalent to the equation $\left(e^{u} X-\operatorname{div}\left(e^{u} X\right)\right) \operatorname{div}\left(e^{u} X\right)=-\lambda^{2}$.) Therefore, it is important to seek out approximate solutions of (E). In the present paper, in order to obtain the approximate solutions, we use a perturbation technique. For this purpose we introduce some notations.

In polar coordinates $(r, \theta)(x=r \cos \theta, y=r \sin \theta)$, system (L) becomes

$$
\left\{\begin{array}{l}
\dot{r}=-\cos \theta f(r \cos \theta), \\
\dot{\theta}=-1+\frac{1}{r} \sin \theta f(r \cos \theta),
\end{array}\right.
$$

and the vector field $X$ is written

$$
X=-\cos \theta f(r \cos \theta) \frac{\partial}{\partial r}+\left(-1+\frac{1}{r} \sin \theta f(r \cos \theta)\right) \frac{\partial}{\partial \theta} .
$$

Put

$$
X_{1}=-\cos \theta f(r \cos \theta) \frac{\partial}{\partial r}+\frac{1}{r} \sin \theta f(r \cos \theta) \frac{\partial}{\partial \theta}
$$

Then we have $X=-\partial / \partial \theta+X_{1}$. For a function $g=g(x, y)$, we write $g_{\theta}$ $=\partial g / \partial \theta, g_{r}=\partial g / \partial r, \cdots$. 
For a continuous function $g$ of angular variable $\theta$, we put

$$
\bar{g}=\frac{1}{2 \pi} \int_{0}^{2 \pi} g(\theta) d \theta
$$

If the mean value $\bar{g}$ vanishes, then we denote by $\int g d \theta$ the primitive function whose mean value vanishes. It is obvious that

$$
\int g d \theta=\int_{0}^{\theta} g d \theta-\frac{1}{2 \pi} \int_{0}^{2 \pi}\left(\int_{0}^{\theta} g d \theta\right) d \theta \text {. }
$$

The assumption $\bar{g}=0$ implies that $\int g d \theta$ is a function of angular variable $\theta$, i.e., a periodic function with period $2 \pi$. For a continuous function $g$ of $\theta$, it is obvious that $\int(g-\bar{g}) d \theta$ is a function of $\theta$. Note that if $g(\theta)$ is odd (i.e., $g(-\theta)=-g(\theta)$ ), then $\int g d \theta$ is defined (and even), because $\bar{g}$ $=0$. If $g(\theta)$ is even and $\bar{g}=0$, then the function $\int_{0}^{\theta} g d \theta$ is odd and hence we have $\int g d \theta=\int_{0}^{\theta} g d \theta$.

Proposition 2.2.1. Assume that $f$ is written in the form

$$
f(x)=\mu f_{1}(x)
$$

where $\mu$ is a constant, and $f_{1}$ is independent of $\mu$. Let $\lambda$ be a real number. Put

$$
a=\frac{1}{2 \pi} \int_{0}^{2 \pi}(\operatorname{div} X) d \theta, \quad b=\frac{1}{2 \pi} \int_{0}^{2 \pi} \cos \theta f(r \cos \theta) d \theta,
$$

and put

$$
\chi_{1}=X_{1}-\operatorname{div} X \text {. }
$$

(We consider $\chi_{1}$ as an operator.) Let $U_{0}(r), U_{2}(r), \cdots, U_{2 n}(r)(n \geqslant 1)$ be $C^{\infty}$ functions depending only on $r$. Define

$$
u=u_{0}+\mu u_{1}+\mu^{2} u_{2}+\cdots+\mu^{2 n} u_{2 n}
$$

as follows.

$$
\begin{gathered}
u_{0}=U_{0}(r) . \\
\mu u_{1}=A-u_{0 r} B,
\end{gathered}
$$


where

$$
A=\int(\operatorname{div} X-a) d \theta, \quad B=\int(\cos \theta f(r \cos \theta)-b) d \theta, \quad u_{0 r}=\frac{d u_{0}}{d r} .
$$

(2) $\mu^{2} u_{2}=\int\left(X_{1} \mu u_{1}\right) d \theta+\left(a-b u_{0 r}\right)_{r} \int B d \theta+\left(a-b u_{0 r}\right) \int A d \theta+\mu^{2} U_{2}(r)$.

In general, for $2 \leqslant m \leqslant n$,

$(2 m-1) \quad \mu^{2 m-1} u_{2 m-1}=\int\left(X_{1} \mu^{2 m-2} u_{2 m-2}-\overline{\left.X_{1} \mu^{2 m-2} u_{2 m-2}\right)} d \theta+\iint\left(\chi_{1} \Phi_{2 m-2}\right) d \theta d \theta\right.$, where

$$
\Phi_{2 m-2}=\left(\mu^{2 m-2} u_{2 m-2}\right)_{\theta}-X_{1}\left(\mu^{2 m-3} u_{2 m-3}\right) .
$$

(2m) $\quad \mu^{2 m} u_{2 m}=\int\left(X_{1} \mu^{2 m-1} u_{2 m-1}\right) d \theta+\iint\left(\chi_{1} \Phi_{2 m-1}-\overline{\chi_{1} \Phi_{2 m-1}}\right) d \theta d \theta+\mu^{2 m} U_{2 m}(r)$, where

$$
\Phi_{2 m-1}=\left(\mu^{2 m-1} u_{2 m-1}\right)_{\theta}-X_{1}\left(\mu^{2 m-2} u_{2 m-2}\right)=-\overline{X_{1} \mu^{2 m-2} u_{2 m-2}}+\int\left(\chi_{1} \Phi_{2 m-2}\right) d \theta .
$$

Then we have:

$$
\begin{aligned}
(X- & \operatorname{div} X) X u+(X-\operatorname{div} X) \operatorname{div} X+\lambda^{2} e^{-2 u} \\
= & b\left(b u_{0 r}-a\right)_{r}+a\left(b u_{0 r}-a\right)+\lambda^{2} e^{-2 u} \\
& -\overline{\chi_{1} \Phi_{3}}-\cdots-\overline{\chi_{1} \Phi_{2 k+1}}-\cdots-\overline{\chi_{1} \Phi_{2 n-1}} \\
& -\left(X_{1} \mu^{2 n} u_{2 n}\right)_{\theta}-\chi_{1} \Phi_{2 n}-\chi_{1}\left(-X_{1} \mu^{2 n} u_{2 n}\right)
\end{aligned}
$$

where

$$
\Phi_{2 n}=\left(\mu^{2 n} u_{2 n}\right)_{\theta}-X_{1}\left(\mu^{2 n-1} u_{2 n-1}\right) .
$$

Proof. For simplicity of notation, we put

$$
E=(X-\operatorname{div} X) X u+(X-\operatorname{div} X) \operatorname{div} X+\lambda^{2} e^{-2 u} .
$$

Recall that $X=-\partial / \partial \theta+X_{1}, \chi_{1}=X_{1}-\operatorname{div} X$. Then we have

$$
\begin{aligned}
E & =\left(-\frac{\partial}{\partial \theta}+\chi_{1}\right)\left(-u_{\theta}+X_{1} u\right)+\left(-\frac{\partial}{\partial \theta}+\chi_{1}\right) \operatorname{div} X+\lambda^{2} e^{-2 u} \\
& =u_{\theta \theta}-\left(X_{1} u\right)_{\theta}-\chi_{1}\left(u_{\theta}-X_{1} u\right)-(\operatorname{div} X)_{\theta}+\chi_{1}(\operatorname{div} X)+\lambda^{2} e^{-2 u}
\end{aligned}
$$

Substituting $u=u_{0}+\mu u_{1}+\cdots+\mu^{2 n} u_{2 n}$ in this expression, and considering the powers of $\mu$, since $u_{0}=U_{0}(r)$, we get 
60

$$
\begin{aligned}
E= & \left(\mu u_{1}\right)_{\theta \theta}-\left(X_{1} u_{0}\right)_{\theta}-(\operatorname{div} X)_{\theta} \\
& +\left(\mu^{2} u_{2}\right)_{\theta \theta}-\left(X_{1} \mu u_{1}\right)_{\theta}-\chi_{1}\left(\mu u_{1 \theta}-X_{1} u_{0}\right)+\chi_{1}(\operatorname{div} X)+\lambda^{2} e^{-2 u} \\
& +\left(\mu^{3} u_{3}\right)_{\theta \theta}-\left(X_{1} \mu^{2} u_{2}\right)_{\theta}-\chi_{1}\left(\mu^{2} u_{2 \theta}-X_{1} \mu u_{1}\right) \\
& +\cdots \\
& +\left(\mu^{2 n} u_{2 n}\right)_{\theta \theta}-\left(X_{1} \mu^{2 n-1} u_{2 n-1}\right)_{\theta}-\chi_{1}\left(\mu^{2 n-1} u_{2 n-1 \theta}-X_{1} \mu^{2 n-2} u_{2 n-2}\right) \\
& -\left(X_{1} \mu^{2 n} u_{2 n}\right)_{\theta}-\chi_{1}\left(\mu^{2 n} u_{2 n \theta}-X_{1} \mu^{2 n-1} u_{2 n-1}\right)-\chi_{1}\left(-X_{1} \mu^{2 n} u_{2 n}\right) .
\end{aligned}
$$

For $1 \leqslant j \leqslant 2 n$, put

$$
\Phi_{j}=\left(\mu^{j} u_{j}\right)_{\theta}-X_{1} \mu^{j-1} u_{j-1}
$$

Then

$$
\begin{aligned}
E= & \Phi_{1 \theta}-(\operatorname{div} X)_{\theta} \\
& +\Phi_{2 \theta}-\chi_{1} \Phi_{1}+\chi_{1}(\operatorname{div} X)+\lambda^{2} e^{-2 u} \\
& +\Phi_{3 \theta}-\chi_{1} \Phi_{2} \\
& +\cdots \\
& +\Phi_{j \theta}-\chi_{1} \Phi_{j-1} \\
& +\cdots \\
& +\Phi_{2 n \theta}-\chi_{1} \Phi_{2 n-1} \\
& -\left(X_{1} \mu^{2 n} u_{2 n}\right)_{\theta}-\chi_{1} \Phi_{2 n}-\chi_{1}\left(-X_{1} \mu^{2 n} u_{2 n}\right)
\end{aligned}
$$

On the other hand, by our definition we see that $\mu^{j} u_{j}$ is an even or odd function of $\theta$ according as $j$ is even or odd, because A, B are clearly odd, and the operators $X_{1}, \chi_{1}$ preserve the property of "even" or "odd". Hence we also see that $\Phi_{j}$ is an even or odd function of $\theta$ according as $j$ is odd or even. Let us return to the expression for $E$ obtained above. Since $\Phi_{1}=\operatorname{div} X-a+b u_{0 r}$, we see that the 1 st term $\Phi_{1 \theta}-(\operatorname{div} X)_{\theta}$ certainly vanishes. It is easy to see that

$$
\Phi_{2 \theta}=-\chi_{1}\left(a-b u_{0 r}\right)+b\left(b u_{0 r}-a\right)_{r}+a\left(b u_{0 r}-a\right) .
$$

Hence the 2 nd term $\Phi_{2 \theta}-\chi_{1}\left(\Phi_{1}-\operatorname{div} X\right)+\lambda^{2} e^{-2 u}$ is equal to

$$
b\left(b u_{0 r}-a\right)_{r}+a\left(b u_{0 r}-a\right)+\lambda^{2} e^{-2 u} .
$$

Now, for $3 \leqslant j \leqslant 2 n$, the definitions of $\mu^{j} u_{j}, \Phi_{j}$ yield

$$
\Phi_{j \theta}=\chi_{1} \Phi_{j-1}-\overline{\chi_{1} \Phi_{j-1}} .
$$

(If $j$ is odd, we have $\overline{\chi_{1} \Phi_{j-1}}=0$, because $\chi_{1} \Phi_{j-1}$ is odd.) Consequently, we see that $E$ is equal to the desired expression. 
Remark. The formula (*) in the proof will be used later.

The following fact can be directly verified.

Proposition 2.2.2. Let $u=u_{0}+\mu u_{1}+\cdots+\mu^{2 n} u_{2 n}$ be as in the preceding proposition. Then we have

$$
\operatorname{div}\left(e^{u} X\right)=e^{u}\left(a-b u_{0 r}-\Phi_{2}-\Phi_{3}-\cdots-\Phi_{2 n}+X_{1} \mu^{2 n} u_{2 n}\right),
$$

where

$$
\Phi_{j}=\left(\mu^{j} u_{j}\right)_{\theta}-X_{1}\left(\mu^{j-1} u_{j-1}\right) .
$$

The following lemma gives us how to define the function $u_{0}$.

Lemma 2.2.3. Let $a, b$ be the same as in Proposition 2.2.1. Assume that $b=b(r)$ has only a finite number of zeroes. Let $b_{0}=0<b_{1}<\cdots<b_{m}$ be the nonnegative zeroes, and assume that $b_{r}\left(b_{i}\right) \neq 0$ for each $b_{i}$, in other words, each $b_{i}$ is a simple root of $b(r)=0$. Let $\kappa, \lambda$ be two positive numbers. Furthermore, assume that $w(x, y)$ depends only on $r=\sqrt{x^{2}+y^{2}}$, and that

$$
\int_{b_{i}^{2} \leqslant x^{2}+y^{2} \leqslant b_{i+1}^{2}} e^{w} d x d y=\kappa \quad \text { for each } i=0,1, \cdots, m,
$$

where $b_{m+1}=\infty$. Suppose that $b(r)<0$ for $r \in\left(0, b_{1}\right)$. Define a function $u_{0}(r)$ by the following formulas:

$$
\begin{aligned}
e^{u_{0}\left(b_{i}\right)} & =\frac{(-1)^{i} \lambda}{a\left(b_{i}\right)} \\
e^{u_{0}(r)} & =-\frac{\kappa \lambda}{2 \pi^{2}} \frac{1}{r e^{w} b} \sin \left(\frac{\pi}{\kappa} \int_{[0, r]} \omega\right) \quad \text { for } r \neq b_{i},
\end{aligned}
$$

where

$$
\int_{[0, r]} \omega=\int_{0 \leqslant x^{2}+y^{2} \leqslant r^{2}} e^{w} d x d y
$$

Then $u_{0}(r)$ is a $C^{\infty}$ function of $r$, and satisfies the differential equation

$$
b\left(b u_{0 r}-a\right)_{r}+a\left(b u_{0 r}-a\right)+\lambda^{2} e^{-2 u_{0}}=0 .
$$

Furthermore we have

$$
e^{u_{0}}\left(a-b u_{0 r}\right)=\lambda \cos \left(\frac{\pi}{\kappa} \int_{[0, r]} \omega\right) .
$$

Remark. In the case where $b(r)>0$ for $r \in\left(0, b_{1}\right)$, we define $u_{0}(r)$ by the formulas: 


$$
\begin{aligned}
e^{u_{0}\left(b_{i}\right)} & =\frac{(-1)^{i+1} \lambda}{a\left(b_{i}\right)}, \\
e^{u_{0}(r)} & =\frac{\kappa \lambda}{2 \pi^{2}} \frac{1}{r e^{w} b} \sin \left(\frac{\pi}{\kappa} \int_{[0, r]} \omega\right) \quad \text { for } r \neq b_{i} .
\end{aligned}
$$

Then $u_{0}(r)$ satisfies the same conclusion as in the lemma, except that the last formula is replaced by

$$
e^{u_{0}}\left(a-b u_{0 r}\right)=-\lambda \cos \left(\frac{\pi}{\kappa} \int_{[0, r]} \omega\right) .
$$

Before proving the lemma, we note the following fact.

Lemma 2.2.4. Let $a, b$ be as in Proposition 2.2.1. Assume that $w(x, y)$ depends only on $r=\sqrt{x^{2}+y^{2}}$. Then we have

$$
\begin{aligned}
& a=-\frac{1}{2 \pi} \int_{0}^{2 \pi} f_{x}(r \cos \theta) d \theta-b w_{r}, \\
& b_{r}=\frac{1}{2 \pi} \int_{0}^{2 \pi} f_{x}(r \cos \theta) d \theta-\frac{b}{r} .
\end{aligned}
$$

Proof. The former relation can be directly verified. By integration by parts, we have

$$
\frac{1}{2 \pi} \int_{0}^{2 \pi} \sin ^{2} \theta f_{x}(r \cos \theta) d \theta=\frac{b}{r}
$$

From this we obtain the latter relation.

Proof of Lemma 2.2.3. To prove the smoothness of $u_{0}(r)$, clearly it suffices to prove the smoothness of $e^{u_{0}(r)}$ at $r=b_{i}$. Let $i \geqslant 1$. Note that the $C^{\infty}$ function $b(r)$ is written

$$
b(r)=(-1)^{i+1}\left(r-b_{i}\right) \beta(r),
$$

where $\beta(r)$ is a $C^{\infty}$ function with $\beta\left(b_{i}\right)>0$. Using Lemma 2.2.4, we see that $\beta\left(b_{i}\right)=(-1)^{i} a\left(b_{i}\right)$. Note that the $C^{\infty}$ function $\sin \left(\pi / \kappa \int_{[0, r]} \omega\right)$ is also. written

$$
\sin \left(\frac{\pi}{\kappa} \int_{[0, r]} \omega\right)=(-1)^{i}\left(r-b_{i}\right) \sigma(r),
$$

where $\sigma(r)$ is a $C^{\infty}$ function with $\sigma\left(b_{i}\right)>0$. Using the fact 


$$
\frac{d}{d r} \int_{[0, r]} \omega=2 \pi r e^{w}
$$

we see that $\sigma\left(b_{i}\right)=\left(2 \pi^{2} / \kappa\right) b_{i} e^{w\left(b_{i}\right)}$. From these expressions it follows that

$$
e^{u_{0}(r)}=\frac{\kappa \lambda}{2 \pi^{2}} \frac{\sigma(r)}{r e^{w} \beta(r)}
$$

on a neighborhood of $r=b_{i}$. This shows that $e^{u_{0}(r)}$ is of class $C^{\infty}$ at $r=b_{i}$. Similarly, we can verify that $e^{u_{0}(r)}$ is of class $C^{\infty}$ at $r=0$. Consequently, we see that $u_{0}(r)$ is a $C^{\infty}$ function. Now, differentiating the both sides of

$$
b e^{u_{0}}=-\frac{\kappa \lambda}{2 \pi^{2}} \frac{1}{r e^{\omega}} \sin \left(\frac{\pi}{\kappa} \int_{[0, r]} \omega\right),
$$

we get

$$
e^{u_{0}}\left(b_{r}+b u_{0 r}\right)=-\frac{\kappa \lambda}{2 \pi^{2}}\left(\frac{1}{r e^{w}}\right)_{r} \sin \left(\frac{\pi}{\kappa} \int_{[0, r]} \omega\right)-\lambda \cos \left(\frac{\pi}{\kappa} \int_{[0, r]} \omega\right) .
$$

The first term of the right-hand side is equal to $-b e^{u_{0}}\left(w_{r}+1 / r\right)$. Since $b_{r}=-a-b\left(w_{r}+1 / r\right)$, we obtain

$$
e^{u_{0}}\left(a-b u_{0 r}\right)=\lambda \cos \left(\frac{\pi}{\kappa} \int_{[0, r]} \omega\right)
$$

which is the latter formula in our lemma. Again differentiating the both sides of this formula, we get

$$
e^{u_{0}}\left\{\left(a-b u_{0 r}\right)_{r}+u_{0 r}\left(a-b u_{0 r}\right)\right\}=\left(b e^{u_{0}}\right)^{-1} \lambda^{2} \sin ^{2}\left(\frac{\pi}{\kappa} \int_{[0, r]} \omega\right) .
$$

Since

$$
b\left(a-b u_{0 r}\right)_{r}+a\left(a-b u_{0 r}\right)=b\left\{\left(a-b u_{0 r}\right)_{r}+u_{0 r}\left(a-b u_{0 r}\right)\right\}+\left(a-b u_{0 r}\right)^{2},
$$

we obtain

$$
\begin{aligned}
b\left(a-b u_{0 r}\right)_{r}+a\left(a-b u_{0 r}\right) & =\lambda^{2} e^{-2 u_{0}}\left\{\sin ^{2}\left(\frac{\pi}{\kappa} \int \omega\right)+\cos ^{2}\left(\frac{\pi}{\kappa} \int \omega\right)\right\} \\
& =\lambda^{2} e^{-2 u_{0}} .
\end{aligned}
$$

This proves our lemma.

By taking $u=u_{0}+\mu u_{1}+\mu^{2} u_{2}$ as an approximate solution of (E), we can now prove the following fact. 
Theorem (Lefschetz [9, p. 320]). Let $f, b$ be the same as in Proposition 2.2.1. Assume that $b=b(r)$ has only a finite number of zeroes, and let $b_{0}=0<b_{1}<\cdots<b_{m}$ be the nonnegative zeroes, and $R$ a number greater than $b_{m}$. Assume that $b_{r}\left(b_{i}\right) \neq 0$ for each $b_{i}$. Then there exists a positive number $\mu_{0}$ such that if $0<|\mu|<\mu_{0}$, then the system (L) on the disk $\left\{(x, y) \mid x^{2}\right.$ $\left.+y^{2} \leqslant R^{2}\right\}$ has precisely $m$ limit cycles. As $\mu \rightarrow 0$, each limit cycle tends to a circle $\left\{(x, y) \mid x^{2}+y^{2}=b_{i}^{2}\right\}$ for some $b_{i}(1 \leqslant i \leqslant m)$.

Proof. Put $D_{R}=\left\{(x, y) \mid x^{2}+y^{2} \leqslant R^{2}\right\}$. It is easy to construct a $C^{\infty}$ function $w(r)$ so that

$$
2 \pi \int_{b_{i}}^{b_{i+1}} r e^{w(r)} d r=1
$$

for $i=0, \cdots, m$, where $b_{m+1}=\infty$. Let $\omega=e^{w(r)} d x d y$, and let $\kappa=1, \lambda=|\mu|$. Then we can apply Lemma 2.2.3 or the remark. Define $u_{0}(r)$ by the formulas in Lemma 2.2 .3 or in the remark according as $b(r)<0$ or $>0$ on $\left(0, b_{1}\right)$. By the definition of $u_{0}(r)$, we have at once

$$
b\left(b u_{0 r}-a\right)_{r}+a\left(b u_{0 r}-a\right)+\mu^{2} e^{-2 u_{0}}=0 .
$$

Note that $u_{0}(r)$ is independent of $\mu$. Let $U_{2}(r) \equiv 0$. Defining $\mu u_{1}, \mu^{2} u_{2}$ by the formulas in Proposition 2.2.1, and putting $u=u_{0}+\mu u_{1}+\mu^{2} u_{2}$, we get

$$
\begin{aligned}
& (X-\operatorname{div} X) X u+(X-\operatorname{div} X) \operatorname{div} X \\
& \quad=b\left(b u_{0 r}-a\right)_{r}+a\left(b u_{0 r}-a\right)-\left(X_{1} \mu^{2} u_{2}\right)_{\theta}-\chi_{1} \Phi_{2}-\chi_{1}\left(-X_{1} \mu^{2} u_{2}\right) .
\end{aligned}
$$

Hence

$$
(X-\operatorname{div} X) X u+(X-\operatorname{div} X) \operatorname{div} X=-\mu^{2} e^{-2 u_{0}}+[\mu]_{3},
$$

where $[\mu]_{3}$ is a function such that

$$
\left|[\mu]_{3}\right| \leqslant K_{3}|\mu|^{3}
$$

for any $(x, y) \in D_{R}$ and any $\mu$ with $|\mu|<1$, where $K_{3}$ is a positive constant. Since $e^{-2 u}=e^{-2 u_{0}}\left(1-2\left(\mu u_{1}+\mu^{2} u_{2}\right)+\cdots\right)$, for each positive integer $n$, we can find a constant $\mu_{n}>0$ such that

$$
-\left(\frac{n+1}{n} \mu\right)^{2} e^{-2 u}<-\mu^{2} e^{-2 u_{0}}+[\mu]_{3}<-\left(\frac{n-1}{n} \mu\right)^{2} e^{-2 u}
$$

for any $(x, y) \in D_{R}$ and any $\mu$ with $0<|\mu|<\mu_{n}$. We may assume that $\mu_{n}<1 / n^{2}$ for each $n$. Consequently we obtain 
$-\left(\frac{n+1}{n} \mu\right)^{2} e^{-2 u}<(X-\operatorname{div} X) X u+(X-\operatorname{div} X) \operatorname{div} X<-\left(\frac{n-1}{n} \mu\right)^{2} e^{-2 u}$

for any $(\mathrm{x}, y) \in D_{R}$ and for any $\mu$ with $0<|\mu|<\mu_{n}$. On the other hand, by Proposition 2.2.2 and Lemma 2.2.3 or the remark, we have

$$
\operatorname{div}\left(e^{u} X\right)= \pm \mu \cos \left(\pi \int_{[0, r]} \omega\right)+[\mu]_{2},
$$

where $[\mu]_{2}$ is a function such that $\left|[\mu]_{2}\right| \leqslant K_{2}|\mu|^{2}$ for any $(x, y) \in D_{R}$ and any $\mu$ with $|\mu|<1$, where $K_{2}$ is a positive constant. From the proof of Theorem 1.2.2 we now observe that if $N$ is a sufficiently large number, then for any $n \geqslant N$ and for any $\mu$ with $0<|\mu| \leqslant \mu_{n}$, the set

$$
M_{n}=\left\{(x, y) \in D_{R}||\left(\operatorname{div} e^{u} X\right)(x, y)\left|\geqslant \frac{n-1}{n}\right| \mu \mid\right\}
$$

is written as a disjoint union

$$
M_{n}=A_{0} \cup A_{1} \cup \cdots \cup A_{m}
$$

where $A_{i}$ satisfy the following conditions:

(i) $A_{0}$ is a closed disk containing the origin, and each $A_{i}(i \geqslant 1)$ is a closed annular domain containing the circle $x^{2}+y^{2}=b_{i}^{2}$.

(ii) Each $A_{i}$ is invariant in the positive or negative direction of $X$.

(iii) Each $A_{i}(i \geqslant 1)$ contains exactly one limit cycle, and any limit cycle in $D_{R}$ is contained in some $A_{i}(i \geqslant 1)$.

(iv) As $n \rightarrow \infty$, each $A_{i}(i \geqslant 1)$ tends to the circle $x^{2}+y^{2}=b_{i}^{2}$.

This proves the theorem.

Remark. The preceding theorem does not give us the qualitative property on the whole plane $R^{2}$. By means of a Liapunov function ([8]), the following fact is known: (J. R. Graef [4, Theorem 3.1]) Assume that there are positive constants $k$ and $c$ such that

$$
f(x) \geqslant c \quad \text { if } x \geqslant k
$$

(and hence $f(x) \leqslant-c$ if $x \leqslant-k$, because $f$ is assumed to be odd). Then there is a closed disk $D$ such that the vector field $X$ points inward along the boundary $\partial D$, and such that any trajectory starting in $R^{2}-D$ enters $D$ after a positive time. 
2.3. The case $f(x)=\mu \sin x$

Let us apply our theory to the system

$$
(\mathrm{L})_{\mu \sin x} \quad\left\{\begin{array}{l}
\dot{x}=y-\mu \sin x, \\
\dot{y}=-x,
\end{array}\right.
$$

where $\mu$ is a constant. Hochstadt, Stephan [6] and D'heedene [3] proved that for $\mu \neq 0$, system $(L)_{\mu \sin x}$ has an infinite number of limit cycles. We shall prove this fact by taking such a function $u=u_{0}+\mu u_{1}+\cdots+\mu^{8} u_{8}$ as in Proposition 2.2.1, by verifying that the system multiplied by $e^{u}$ satisfies condition $\left(\mathrm{I}^{\prime}\right)$ in Theorem 2.1.2 (in a neighborhood at $\infty$ ), and by observing that the set $\Sigma$ contains an infinite number of circles.

Now let $f(x)=\mu \sin x$, and recall the notation in 2.2. The vector field $X$ corresponding to system $(\mathrm{L})_{\mu \sin x}$ is written in polar coordinates $(r, \theta)$

$$
X=-\frac{\partial}{\partial \theta}+X_{1}
$$

where

$$
X_{1}=-\cos \theta f(r \cos \theta) \frac{\partial}{\partial r}+\frac{1}{r} \sin \theta f(r \cos \theta) \frac{\partial}{\partial \theta} .
$$

For a function $g$ of angular variable $\theta$, we denote by $\bar{g}$ its mean value.

Proposition 2.3.1. Let $\omega=d x d y$ and let $\mu \neq 0$. Let $u_{0}=U_{0}(r)$ be $a$ $C^{\infty}$ function such that

$$
u_{0}=\frac{1}{2} \log \frac{\pi r}{2} \quad \text { for } r \geqslant 1,
$$

and let $U_{2}(r)=U_{4}(r)=\cdots=U_{8}(r) \equiv 0$. Define $\mu u_{1}, \mu^{2} u_{2}, \cdots, \mu^{8} u_{8}$ by the formulas in Proposition 2.2.1. Put $u=u_{0}+\mu u_{1}+\cdots+\mu^{8} u_{8}$. If $\varepsilon$ is $a$ sufficiently small positive number, then there exists a positive number $R$ such that the function $u$ satisfies

$$
(X-\operatorname{div} X) X u+(X-\operatorname{div} X) \operatorname{div} X+\varepsilon^{2} e^{-2 u}<0
$$

on $\left\{(x, y) \mid r=\sqrt{x^{2}+y^{2}}>R\right\}$.

For the proof we use the notation $O$ in the following sense: for a function $g(r, \theta)$ which is not assumed to have period $2 \pi$ in the angular variable $\theta$, we write 


$$
g(r, \theta)=O\left(r^{-m}\right)
$$

if there exists a constant $K$ such that $r^{m}|g(r, \theta)| \leqslant K$ for any $r \geqslant 1$ and for any $\theta$ satisfying $0 \leqslant \theta \leqslant 2 \pi$.

LEMMA 2.3.2. The functions

$$
a=-\frac{1}{2 \pi} \int_{0}^{2 \pi} f_{x}(r \cos \theta) d \theta, \quad b=\frac{1}{2 \pi} \int_{0}^{2 \pi} \cos \theta f(r \cos \theta) d \theta
$$

have the following properties:

(i ) $a=-\mu J_{0}(r), b=\mu J_{1}(r)$, where $J_{0}(r), J_{1}(r)$ are the Bessel functions of order 0 , order 1 respectively.

(ii) $a_{r}=b, b_{r}=-a-\frac{b}{r}$.

(iii) $a=O\left(r^{-\frac{1}{2}}\right), b=O\left(r^{-\frac{1}{2}}\right)$

(iv) $a^{2}+b^{2}=\mu^{2}\left\{\frac{2}{\pi r}+O\left(r^{-2}\right)\right\}$

Proof. The properties (i)-(iii) are well known in the theory of Bessel functions. From the asymptotic expansions of $J_{0}, J_{1}$ we have (iv).

LEMMA 2.3.3. The functions

$$
A=\int_{0}^{\theta}\left(-f_{x}(r \cos \theta)-a\right) d \theta, \quad B=\int_{0}^{\theta}(\cos \theta f(r \cos \theta)-b) d \theta
$$

have the following properties:

(i) $A_{r}=B, B_{r}=-A-\frac{1}{r} B+\frac{1}{r} \sin \theta f$

(ii) $A=O\left(r^{-\frac{1}{2}}\right), B=O\left(r^{-\frac{1}{2}}\right)$.

Proof. It is easy to verify (i). The proof of (ii) is the same as that of Lemma 1 of Hochstadt and Stephan [6, p. 372].

Lemma 2.3.4. The function

$$
\Phi_{2}=\mu^{2} u_{2 \theta}-X_{1}\left(\mu u_{1}\right)
$$

has the following property: For any integer $i \geqslant 0$ we have

$$
\frac{\partial^{i}}{\partial r^{i}} \Phi_{2}=O\left(r^{-1}\right), \quad \frac{\partial^{i+1}}{\partial r^{i} \partial \theta} \Phi_{2}=O\left(r^{-\frac{1}{2}}\right), \quad \frac{\partial^{i+2}}{\partial r^{i} \partial \theta^{2}} \Phi_{2}=O\left(r^{\frac{1}{2}}\right) .
$$

Proof. By the definition of $u_{2}$, we have 


$$
\Phi_{2}=\left(a-b u_{0 r}\right)_{r} B+\left(a-b u_{0 r}\right) A
$$

and hence

$$
\begin{gathered}
\Phi_{2 \theta}=\left(a-b u_{0 r}\right)_{r}(\cos \theta f-b)+\left(a-b u_{0 r}\right)\left(-f_{x}-a\right) \\
\Phi_{2 \theta \theta}=\left(a-b u_{0 r}\right)_{r}\left(-\sin \theta f-r \sin \theta \cos \theta f_{x}\right)+\left(a-b u_{0 r}\right)\left(r \sin \theta f_{x x}\right) .
\end{gathered}
$$

Recall that $u_{0}=\frac{1}{2} \log \pi r / 2$. Hence $u_{0 r}=1 / 2 r$. Using (ii), (iii) of Lemma 2.3.2, we observe that for any integer $i \geqslant 0$,

$$
\frac{\partial^{i}}{\partial r^{i}}\left(a-b u_{0 r}\right)=O\left(r^{-\frac{1}{2}}\right) \text {. }
$$

On the other hand, by (i), (ii) of Lemma 2.3 .3 we also have

$$
\frac{\partial^{i}}{\partial r^{i}} A=O\left(r^{-\frac{1}{2}}\right), \quad \frac{\partial^{i}}{\partial r^{i}} B=O\left(r^{-\frac{1}{2}}\right)
$$

Consequently, using Leibniz's formula we conclude that

$$
\frac{\partial^{i}}{\partial r^{i}} \Phi_{2}=O\left(r^{-1}\right)
$$

for each integer $i \geqslant 0$. Similarly we can verify the others.

LEMma 2.3.5. The function

$$
\mu^{2} u_{2}=\int\left(X_{1} \mu u_{1}\right) d \theta+\left(a-b u_{0 r}\right)_{r} \int B d \theta+\left(a-b u_{0 r}\right) \int A d \theta
$$

has the following property: For any integer $i \geqslant 0$ we have

$$
\frac{\partial^{i}}{\partial r^{i}} \mu^{2} u_{2}=O\left(r^{-1}\right), \quad \frac{\partial^{i+1}}{\partial r^{i} \partial \theta} \mu^{2} u_{2}=O\left(r^{-\frac{1}{2}}\right), \quad \frac{\partial^{i+2}}{\partial r^{i} \partial \theta^{2}} \mu^{2} u_{2}=O\left(r^{\frac{1}{2}}\right) .
$$

Proof. First we verify that

$$
\int A d \theta=O\left(r^{-\frac{1}{2}}\right), \quad \int B d \theta=O\left(r^{-\frac{1}{2}}\right) .
$$

By definition we have

$$
\int A d \theta=\int_{0}^{\theta} A d \theta-\frac{1}{2 \pi} \int_{0}^{2 \pi}\left(\int_{0}^{\theta} A d \theta\right) d \theta
$$

Using the fact $A=O\left(r^{-\frac{1}{2}}\right)$ we obtain $\int A d \theta=O\left(r^{-\frac{1}{2}}\right)$. Similarly we obtain $\int B d \theta=O\left(r^{-\frac{1}{2}}\right)$. Furthermore, using (i) of Lemma 2.3.3, we get 


$$
\frac{\partial^{i}}{\partial r^{i}} \int A d \theta=O\left(r^{-\frac{1}{2}}\right), \quad \frac{\partial^{i}}{\partial r^{i}} \int B d \theta=O\left(r^{-\frac{1}{2}}\right)
$$

for each $i \geqslant 0$. Consequently the function

$$
g=\left(a-b u_{0 r}\right)_{r} \int B d \theta+\left(a-b u_{0 r}\right) \int A d \theta
$$

satisfies $\left(\partial^{i} / \partial r^{i}\right) g=O\left(r^{-1}\right)$. It is easy to see that $g$ satisfies also

$$
\frac{\partial^{i+1}}{\partial r^{i} \partial \theta} g=O\left(r^{-1}\right), \quad \frac{\partial^{i+2}}{\partial r^{i} \partial \theta^{2}} g=O\left(r^{-\frac{1}{2}}\right) .
$$

Therefore, to prove our lemma we have to prove that the function $\int\left(X_{1} \mu u_{1}\right) d \theta$ satisfies

$$
\frac{\partial^{i}}{\partial r^{i}} \int\left(X_{1} \mu u_{1}\right) d \theta=O\left(r^{-1}\right), \quad \frac{\partial^{i}}{\partial r^{i}}\left(X_{1} \mu u_{1}\right)=O\left(r^{-\frac{1}{2}}\right), \quad \frac{\partial^{i+1}}{\partial r^{i} \partial \theta}\left(X_{1} \mu u_{1}\right)=O\left(r^{\frac{1}{2}}\right) .
$$

To prove this, recall that $\mu u_{1}=A-u_{0 r} B$. Since $A_{r}=B$, we have

$$
X_{1} \mu u_{1}=-\cos \theta f\left\{B-\left(u_{0 r} B\right)_{r}\right\}+\frac{\sin \theta f}{r}\left\{-f_{x}-a-u_{0 r}(\cos \theta f-b)\right\} .
$$

Recall that $u_{0 r}=1 / 2 r$. Hence, to prove $\partial^{i} / \partial r^{i} \int\left(X_{1} \mu u_{1}\right) d \theta=O\left(r^{-1}\right)$ it suffices to prove

$$
\frac{\partial^{i}}{\partial r^{i}} \int \cos \theta f B d \theta=O\left(r^{-1}\right)
$$

Note that

$$
\int \cos \theta f B d \theta=\int(\cos \theta f-b) B d \theta+b \int B d \theta=\frac{1}{2} B^{2}+b \int B d \theta .
$$

This shows that $\int \cos \theta f B d \theta=O\left(r^{-1}\right)$. Furthermore, using (i) of Lemma 2.3.3 we observe that $\partial^{i} / \partial r^{i} \int \cos \theta f B d \theta=O\left(r^{-1}\right)$, as desired. It is easy to see that $\left(\partial^{i} / \partial r^{i}\right)\left(X_{1} \mu u_{1}\right)=O\left(r^{-\frac{1}{2}}\right), \quad\left(\partial^{i+1} / \partial r^{i} \partial \theta\right)\left(X_{1} \mu u_{1}\right)=O\left(r^{\frac{1}{2}}\right)$. Our lemma is proved.

LEMMA 2.3.6. Let $g(r, \theta)$ be a $C^{\infty}$ function which is not assumed to have period $2 \pi$ in the angular variable $\theta$. Let $G(r, \theta)$ be the function 
$\int_{0}^{\theta}\left(X_{1} g\right) d \theta$ or the function $\int_{0}^{\theta}\left(\chi_{1} g\right) d \theta$.

(i) Assume that for any integer $i \geqslant 0$, we have

$$
\frac{\partial^{i}}{\partial r^{i}} g=O\left(r^{-m}\right), \quad \frac{\partial^{i+1}}{\partial r^{i} \partial \theta} g=O\left(r^{-m+1 / 2}\right), \quad \frac{\partial^{i+2}}{\partial r^{i} \partial \theta^{2}} g=O\left(r^{-m+3 / 2}\right) .
$$

Then for any integer $i \geqslant 0$ we have

$$
\frac{\partial^{i}}{\partial r^{i}} G=O\left(r^{-m}\right), \quad \frac{\partial^{i+1}}{\partial r^{i} \partial \theta} G=O\left(r^{-m}\right), \quad \frac{\partial^{i+2}}{\partial r^{i} \partial \theta^{2}} G=O\left(r^{-m+1}\right) .
$$

(ii) Assume that for any integer $i \geqslant 0$, we have

$$
\frac{\partial^{i}}{\partial r^{i}} g=O\left(r^{-m}\right), \quad \frac{\partial^{i+1}}{\partial r^{i} \partial \theta} g=O\left(r^{-m}\right), \quad \frac{\partial^{i+2}}{\partial r^{i} \partial \theta^{2}} g=O\left(r^{-m+1}\right) .
$$

Then for any integer $i \geqslant 0$ we have

$$
\frac{\partial^{i}}{\partial r^{i}} G=O\left(r^{-m-\frac{1}{2}}\right), \quad \frac{\partial^{i+1}}{\partial r^{i} \partial \theta} G=O\left(r^{-m}\right), \quad \frac{\partial^{i+2}}{\partial r^{i} \partial \theta^{2}} G=O\left(r^{-m+1}\right) .
$$

Proof. (i) Let $G(r, \theta)=\int_{0}^{\theta}\left(\chi_{1} g\right) d \theta$. By the definition of $\chi_{1}$, we have $G(r, \theta)=-\int_{0}^{\theta} \cos \theta f g_{r} d \theta+\frac{1}{r} \int_{0}^{\theta} \sin \theta f g_{\theta} d \theta-\int_{0}^{\theta}(\operatorname{div} X) g d \theta$

First, we shall prove that the function $G_{1}=\int_{0}^{\theta} \cos \theta f g_{r} d \theta$ satisfies

$$
\frac{\partial^{i}}{\partial r^{i}} G_{1}=O\left(r^{-m}\right), \quad \frac{\partial^{i+1}}{\partial r^{i} \partial \theta} G_{1}=O\left(r^{-m}\right), \quad \frac{\partial^{i+2}}{\partial r^{i} \partial \theta^{2}} G_{1}=O\left(r^{-m+1}\right) .
$$

It is obvious that $\left(\partial^{i} / \partial r^{i}\right) f=O\left(r^{0}\right)$. Hence, by the assumption $\left(\partial^{i} / \partial r^{i}\right) g=$ $O\left(r^{-m}\right)$ we see that $G_{1}$ satisfies the first two properties. Since

$$
\frac{\partial^{i+2}}{\partial r^{i} \partial \theta^{2}} G_{1}=\frac{\partial^{i}}{\partial r^{i}}\left\{-\sin \theta f g_{r}+\cos \theta\left(-r \sin \theta f_{x}\right) g_{r}+\cos \theta f g_{r \theta}\right\},
$$

by the assumption $\left(\partial^{i+1} / \partial r^{i} \partial \theta\right) g=O\left(r^{-m+\frac{1}{2}}\right)$ we see that $G_{1}$ satisfies also the last property. Similarly we observe that the function $\int_{0}^{\theta}(\operatorname{div} X) g d \theta$ satisfies the same property as $G_{1}$. Next, we shall prove that the function $G_{2}=$ $1 / r \int_{0}^{\theta} \sin \theta f g_{\theta} d \theta$ satisfies 


$$
\frac{\partial^{i}}{\partial r^{i}} G_{2}=O\left(r^{-m-\frac{1}{2}}\right), \quad \frac{\partial^{i+1}}{\partial r^{i} \partial \theta} G_{2}=O\left(r^{-m-\frac{1}{2}}\right), \quad \frac{\partial^{i+2}}{\partial r^{i} \partial \theta^{2}} G_{2}=O\left(r^{-m+\frac{1}{2}}\right)
$$

Since $\left(\partial^{i+1} / \partial r^{i} \partial \theta\right) g=O\left(r^{-m+\frac{1}{2}}\right)$, the function $G_{2}$ satisfies the first two properties. Since

$$
\frac{\partial^{i+2}}{\partial r^{i} \partial \theta^{2}} G_{2}=\frac{\partial^{i}}{\partial r^{i}} \frac{1}{r}\left\{\cos \theta f g_{\theta}+\sin \theta(-r \sin \theta) f_{x} g_{\theta}+\sin \theta f g_{\theta \theta}\right\},
$$

and $\left(\partial^{i+2} / \partial r^{i} \partial \theta^{2}\right) g=O\left(r^{-m+3 / 2}\right)$, we obtain $\left(\partial^{i+2} / \partial r^{i} \partial \theta^{2}\right) G_{2}=O\left(r^{-m+\frac{1}{2}}\right)$. In particular, the function $G_{2}$ satisfies the same property as $G_{1}$. From these we conclude that the function $\int_{0}^{\theta}\left(\chi_{1} g\right) d \theta$ satisfies the desired property. The proof above shows that the function $\int_{0}^{\theta}\left(X_{1} g\right) d \theta$ also satisfies the same property. Part (i) is proved.

(ii) As in the proof of (i), let $G_{1}=\int_{0}^{\theta} \cos \theta f g_{r} d \theta$. We shall prove that

$$
\frac{\partial^{i}}{\partial r^{i}} G_{1}=O\left(r^{-m-\frac{1}{2}}\right), \quad \frac{\partial^{i+1}}{\partial r^{i} \partial \theta} G_{1}=O\left(r^{-m}\right), \quad \frac{\partial^{i+2}}{\partial r^{i} \partial \theta^{2}} G_{1}=O\left(r^{-m+1}\right)
$$

By integration by parts we have

$$
\frac{\partial^{i}}{\partial r^{i}} G_{1}=\frac{\partial^{i}}{\partial r^{i}}\left(B g_{r}-\int_{0}^{\theta} B g_{r \theta} d \theta+b \int_{0}^{\theta} g_{r} d \theta\right) \text {. }
$$

Note that $\left(d^{i} / d r^{i}\right) b=O\left(r^{-\frac{1}{2}}\right)$ because of (ii), (iii) of Lemma 2.3.2, and note that $\left(\partial^{i} / \partial r^{i}\right) B=O\left(r^{-\frac{1}{2}}\right)$ because of (i), (ii) of Lemma 2.3.3. Then the assumptions $\left(\partial^{i} / \partial r^{i}\right) g=O\left(r^{-m}\right),\left(\partial^{i+1} / \partial r^{i} \partial \theta\right) g=O\left(r^{-m}\right)$ yield $\left(\partial^{i} / \partial r^{i}\right) G_{1}=O\left(r^{-m-\frac{1}{2}}\right)$. Since $\left(\partial^{i+1} / \partial r^{i} \partial \theta\right) G_{1}=\left(\partial^{i} / \partial r^{i}\right)\left(\cos \theta f g_{r}\right)$, we have at once $\left(\partial^{i+1} / \partial r^{i} \partial \theta\right) G_{1}=O\left(r^{-m}\right)$. Further, since

$$
\frac{\partial^{i+2}}{\partial r^{i} \partial \theta^{2}} G_{1}=\frac{\partial^{i}}{\partial r^{i}}\left\{-\sin \theta f g_{r}+\cos \theta\left(-r \sin \theta f_{x}\right) g_{r}+\cos \theta f g_{r \theta}\right\}
$$

we obtain $\left(\partial^{i+2} / \partial r^{i} \partial \theta^{2}\right) G_{1}=O\left(r^{-m+1}\right)$. Similarly we observe that the function $\int_{0}^{\theta}(\operatorname{div} X) g d \theta$ satisfies the same property as $G_{1}$. It is easy to see that the function $G_{2}=1 / r \int_{0}^{\theta} \sin \theta f g_{\theta} d \theta$ satisfies

$$
\frac{\partial^{i}}{\partial r^{i}} G_{2}=O\left(r^{-m-1}\right), \quad \frac{\partial^{i+1}}{\partial r^{i} \partial \theta} G_{2}=O\left(r^{-m-1}\right), \quad \frac{\partial^{i+2}}{\partial r^{i} \partial \theta^{2}} G_{2}=O\left(r^{-m}\right) .
$$


In particular, the function $G_{2}$ satisfies the same property as $G_{1}$. Consequently we see that the functions $\int_{0}^{\theta} X_{1} g d \theta, \int_{0}^{\theta} \chi_{1} g d \theta$ satisfy the same property as $G_{1}$. This proves (ii).

Proof of Proposition 2.3.1. We use the notation in Proposition 2.2.1. We shall prove that

(i) If $\varepsilon$ is a sufficiently small positive number, there exist positive constants $K, R_{0}$ such that

$$
b\left(b u_{0 r}-a\right)_{r}+a\left(b u_{0 r}-a\right)+\varepsilon^{2} e^{-2 u}<-\frac{K}{r}
$$

for all $r>R_{0}$.

(ii) $\overline{\chi_{1} \Phi_{3}}=O\left(r^{-3 / 2}\right), \overline{\chi_{1} \Phi_{5}}=O\left(r^{-2}\right), \overline{\chi_{1} \Phi_{7}}=O\left(r^{-5 / 2}\right)$.

(iii) $\left(X_{1} \mu^{8} u_{8}\right)_{\theta}=O\left(r^{-3 / 2}\right), \chi_{1} \Phi_{8}=O\left(r^{-5 / 2}\right)$.

(iv) $\chi_{1}\left(X_{1} \mu^{8} u_{8}\right)=O\left(r^{-5 / 2}\right)$.

These and Proposition 2.2.1 will prove our proposition. In order to prove our assertion, it is convenient to introduce the following notation: Let $g(r, \theta)$ be a function which is not assumed to have period $2 \pi$ in the angular variable $\theta$ and which is of class $C^{\infty}$ on $\{(r, \theta) \mid r>0\}$. Let $\alpha, \beta$ be two real numbers $\geqslant 0$. We write

$$
g(r, \theta)=O\left(r^{-m} ; \alpha, \beta\right)
$$

if for any integer $i \geqslant 0$ the function $g$ satisfies

$$
\frac{\partial^{i}}{\partial r^{i}} g=O\left(r^{-m}\right), \quad \frac{\partial^{i+1}}{\partial r^{i} \partial \theta} g=O\left(r^{-m+\alpha}\right), \quad \frac{\partial^{i+2}}{\partial r^{i} \partial \theta^{2}} g=O\left(r^{-m+\alpha+\beta}\right) .
$$

Lemmas 2.3.4 and 2.3.5 assert that

$$
\Phi_{2}=O\left(r^{-1} ; \frac{1}{2}, 1\right), \quad \mu^{2} u_{2}=O\left(r^{-1} ; \frac{1}{2}, 1\right) .
$$

Furthermore, by Lemma 2.3.6 we have:

(2) $\quad \int_{0}^{\theta}\left(X_{1} g\right) d \theta, \int_{0}^{\theta}\left(\chi_{1} g\right) d \theta=\left\{\begin{array}{lr}O\left(r^{-m} ; 0,1\right) & \text { if } g(r, \theta)=O\left(r^{-m} ; \frac{1}{2}, 1\right), \\ O\left(r^{-m-\frac{1}{2}} ; \frac{1}{2}, 1\right) & \text { if } g(r, \theta)=O\left(r^{-m} ; 0,1\right) .\end{array}\right.$

We shall also use the following obvious fact: If $G(r, \theta)=O\left(r^{-m} ; \alpha, \beta\right)$, then $\int_{0}^{\theta} G(r, \theta) d \theta=O\left(r^{-m} ; \alpha, \beta\right)$, because we assume that $\alpha, \beta \geqslant 0$. We now contend that 


$$
\begin{gathered}
\Phi_{3}, \mu^{3} u_{3}=O\left(r^{-1} ; 0,1\right), \\
\Phi_{4}, \mu^{4} u_{4}=O\left(r^{-3 / 2} ; \frac{1}{2}, 1\right), \\
\Phi_{5}, \mu^{5} u_{5}=O\left(r^{-3 / 2} ; 0,1\right), \\
\Phi_{6}, \mu^{6} u_{6}=O\left(r^{-2} ; \frac{1}{2}, 1\right), \\
\Phi_{7}, \mu^{7} u_{7}=O\left(r^{-2} ; 0,1\right), \\
\Phi_{8}, \mu^{8} u_{8}=O\left(r^{-5 / 2} ; \frac{1}{2}, 1\right) .
\end{gathered}
$$

Indeed, by definition we have

$$
\begin{aligned}
\Phi_{3} & =-\overline{X_{1} \mu^{2} u_{2}}+\int\left(\chi_{1} \Phi_{2}\right) d \theta \\
\mu^{3} u_{3} & =\int\left(X_{1} \mu^{2} u_{2}-\overline{X_{1} \mu^{2} u_{2}}\right) d \theta+\iint\left(\chi_{1} \Phi_{2}\right) d \theta d \theta .
\end{aligned}
$$

By (1), (2) we see at once that $\Phi_{3}, \mu^{3} u_{3}=O\left(r^{-1} ; 0,1\right)$. The formulas in (3) are proved. By definition we have

$$
\begin{aligned}
\Phi_{4} & =\int\left(\chi_{1} \Phi_{3}-\overline{\chi_{1} \Phi_{3}}\right) d \theta \\
\mu^{4} u_{4} & =\int\left(X_{1} \mu^{3} u_{3}\right) d \theta+\iint\left(\chi_{1} \Phi_{3}-\overline{\chi_{1} \Phi_{3}}\right) d \theta d \theta .
\end{aligned}
$$

By (2), (3) we obtain at once $\Phi_{4}, \mu^{4} u_{4}=O\left(r^{-3 / 2} ; \frac{1}{2}, 1\right)$. Similarly we can verify (5)-(8). Our contention is proved. We can now prove our assertion (i)-(iv). To prove (i), note that the function $\mu u_{1}+\mu^{2} u_{2}+\cdots+\mu^{8} u_{8}$ is bounded on $\left\{(x, y) \mid r^{2}=x^{2}+y^{2} \geqslant 1\right\}$. Using Lemma 2.3.2 we get

$$
b\left(b u_{0 r}-a\right)_{r}+a\left(b u_{0 r}-a\right)+\varepsilon^{2} e^{-2 u}=\frac{2}{\pi r}\left\{-\mu^{2}+\varepsilon^{2} e^{-2 \tilde{u}}+O\left(r^{-1}\right)\right\},
$$

where $\tilde{u}=\mu u_{1}+\cdots+\mu^{8} u_{8}$. Since $e^{-2 \tilde{u}}$ is bounded on $\left\{(x, y) \mid x^{2}+y^{2} \geqslant 1\right\}$, we obtain (i). To prove (ii), we use again (2). Indeed, by (2), (3) we have at once $\overline{\chi_{1} \Phi_{3}}=O\left(r^{-3 / 2} ; \frac{1}{2}, 1\right)$. This shows that $\overline{\chi_{1} \Phi_{3}}=O\left(r^{-3 / 2}\right)$. Similarly we get $\overline{\chi_{1} \Phi_{5}}=O\left(r^{-2}\right), \overline{\chi_{1} \Phi_{7}}=O\left(r^{-5 / 2}\right)$. In order to prove that $\left(X_{1} \mu^{8} u_{8}\right)_{\theta}=O\left(r^{-3 / 2}\right)$, we note that

$$
\int_{0}^{\theta}\left(X_{1} \mu^{8} u_{8}\right) d \theta=O\left(r^{-5 / 2} ; 0,1\right)
$$

because of (2) and (8). Then by definition we get what we want. To prove the latter part of (iii) we use again (2) and (8). Then 


$$
\int_{0}^{\theta} \chi_{1} \Phi_{8} d \theta=O\left(r^{-5 / 2} ; 0,1\right)
$$

and hence, in particular, $\chi_{1} \Phi_{8}=O\left(r^{-5 / 2}\right)$. It remains to prove (iv). The formula (9) yields

$$
X_{1} \mu^{8} u_{8}=O\left(r^{-5 / 2}\right), \quad\left(X_{1} \mu^{8} u_{8}\right)_{r}=O\left(r^{-5 / 2}\right), \quad\left(X_{1} \mu^{8} u_{8}\right)_{\theta}=O\left(r^{-3 / 2}\right) .
$$

From these it follows that $\chi_{1}\left(X_{1} \mu^{8} u_{8}\right)=O\left(r^{-5 / 2}\right)$. This completes the proof of our assertion (i)-(iv). Consequently our proposition is proved.

Proposition 2.3.7. Let $\omega=d x d y$, and $\mu \neq 0$. Let $u=u_{0}+\mu u_{1}+\cdots$ $+\mu^{8} u_{8}$ be the same as in Proposition 2.3.1. Then we have

$$
\operatorname{div}\left(e^{u} X\right)=\mu e^{u}\left\{-\sqrt{\frac{2}{\pi r}} \cos \left(r-\frac{\pi}{4}\right)+O\left(r^{-1}\right)\right\} .
$$

Hence there exists a compact set $K$ in $R^{2}$ such that the set

$$
\Sigma_{K}=\left\{(x, y) \in R^{2}-K \mid\left(\operatorname{div} e^{u} X\right)(x, y)=0\right\}
$$

consists of an infinite number of circles.

Proof. From Proposition 2.2.2 and (1), (3)-(9) in the proof of Proposition 2.3.1 it follows that $\operatorname{div}\left(e^{u} X\right)=e^{u}\left(\alpha+\mu O\left(r^{-1}\right)\right)$. By (i) of Lemma 2.3.2 we have $\operatorname{div}\left(e^{u} X\right)=e^{u}\left(-\mu J_{0}(r)+\mu O\left(r^{-1}\right)\right)$. It is well known that

$$
J_{0}(r)=\sqrt{\frac{2}{\pi r}} \cos \left(r-\frac{\pi}{4}\right)+O\left(r^{-3 / 2}\right) .
$$

This shows the former part of our proposition. The latter part is obvious.

By Propositions 2.3.1, 2.3.7 and from the proof of Theorem 2.1.2 we now conclude that if $\mu \neq 0$, then system $(L)_{\mu \sin x}$ has an infinite number of limit cycles. More precisely, we have:

Theorem (cf. Hochstadt and Stephan [6], D'heedene [3]). Let $\mu \neq 0$. Then there exists a compact set $K$ such that the system $(\mathrm{L})_{\mu \sin x}$ restricted on $\boldsymbol{R}^{2}-K$ has the following properties:

(i) It has an infinite number of closed integral curves.

(ii) Each closed integral curve is positively or negatively asymptotically stable.

Remark. For generalizations of this result, see Comstock [2], Ponzo and Wax [11]. 
2.4. Liénard's equations with a unique limit cycle

It is well known that if $\mu$ is a positive constant, then the van der Pol equation

$$
(\mathrm{L})_{\mu\left(x^{3}-x\right)} \quad\left\{\begin{array}{l}
\dot{x}=y-\mu\left(x^{3}-x\right), \\
\dot{y}=-x
\end{array}\right.
$$

has a unique limit cycle. The remark at the end of 2.1 asserts that if $\mu>0$, there exists a function $u: R^{2}-\{(0,0)\} \rightarrow \boldsymbol{R}$ such that the divergence of the system

$$
\left\{\begin{array}{l}
\dot{x}=\left\{y-\mu\left(x^{3}-x\right)\right\} e^{u} \\
\dot{y}=-x e^{u}
\end{array}\right.
$$

on $\boldsymbol{R}^{2}-\{(0,0)\}$ (with respect to the ordinary area-element $d x d y$ ) is a negative constant. It is important to know the analytic expression of such a function $u$. For instance, it will provide an effective tool in the study of forced van der Pol equations. Here, we shall give explicitly a function $w$ such that if $0<|\mu| \leqslant 1$, then the divergence $\operatorname{div} X$ of the system $(\mathrm{L})_{\mu\left(x^{3}-x\right)}$ with respect to the area-element $\omega=e^{w} d x d y$ satisfies

$$
|\operatorname{div} X|>\text { positive constant }
$$

on $\boldsymbol{R}^{2}-\{(0,0)\}$. This and Theorem 2.4.1 mentioned below will prove that if $0<|\mu| \leqslant 1$, the system $(\mathrm{L})_{\mu\left(x^{3}-x\right)}$ has a unique limit cycle.

Remark. Let $X$ and $w$ be a vector field and a function on $\boldsymbol{R}^{2}$ respectively. The relation between the divergence $\operatorname{div}_{0}\left(e^{w} X\right)$ of $e^{w} X$ with respect to the ordinary area-element $d x d y$ and the divergence $\operatorname{div}_{w} X$ of $X$ with respect to $\omega=e^{w} d x d y$ is given by the formula:

$$
\operatorname{div}_{0}\left(e^{w} X\right)=e^{w} \operatorname{div}_{w} X .
$$

We now begin by noticing the following fact corresponding to Theorem 2.1.4. As in 2.1, let $f(x)$ be a $C^{\infty}$ odd function and let

$$
X=(y-f) \frac{\partial}{\partial x}-x \frac{\partial}{\partial y} .
$$

Theorem 2.4.1. Suppose that $f_{x}(0) \neq 0$. Assume that there exists a $C^{1}$ function $w: \boldsymbol{R}^{2}-\{(0,0)\} \rightarrow \boldsymbol{R}$ and a positive constant $\varepsilon$ such that the divergence $\operatorname{div} X$ of $X$ with respect to $\omega=e^{w} d x d y$ satisfies the following inequality:

$$
\operatorname{div} X<-\varepsilon \text { or } \operatorname{div} X>\varepsilon
$$


on $R^{2}-\{(0,0)\}$ according as $f_{x}(0)<0$ or $f_{x}(0)>0$. If $\int_{R^{2}-D} \omega=\infty$ where $D=\left\{(x, y) \mid x^{2}+y^{2} \leqslant 1\right\}$, then there exists a unique closed integral curve for (L). This integral curve is positively or negatively asymptotically stable according as $f_{x}(0)<0$ or $f_{x}(0)>0$.

The proof is the same as that of Theorem 2.1.4. In order that we may apply this theorem, we prepare the following proposition. We use the notation in 2.2 .

Proposition 2.4.2. Suppose that $f$ is written in the form

$$
f(x)=\mu f_{1}(x),
$$

where $\mu$ is a constant, and $f_{1}$ is independent of $\mu$. Put

$$
a=\frac{-1}{2 \pi} \int_{0}^{2 \pi} f_{x}(r \cos \theta) d \theta, \quad b=\frac{1}{2 \pi} \int_{0}^{2 \pi} \cos \theta f(r \cos \theta) d \theta .
$$

Let $W_{0}(r), W_{2}(r), \cdots, W_{2 n}(r)(n \geqslant 1)$ be functions defined on $r>0$ and of class $C^{\infty}$. Define a function

$$
w=w_{0}+\mu w_{1}+\cdots+\mu^{2 n} w_{2 n}
$$

on $\boldsymbol{R}^{2}-\{(0,0)\}$ as follows.

$$
\begin{gathered}
w_{0}=W_{0}(r) . \\
\mu w_{1}=A-w_{0 r} B,
\end{gathered}
$$

where

$$
A=\int\left(-f_{x}(r \cos \theta)-a\right) d \theta, \quad B=\int(\cos \theta f(r \cos \theta)-b) d \theta .
$$

$$
\mu^{2} w_{2}=\int\left(X_{1} \mu w_{1}\right) d \theta+\mu^{2} W_{2}(r)
$$

In general, for $2 \leqslant m \leqslant n$,

$(2 m-1)$

$$
\begin{aligned}
\mu^{2 m-1} w_{2 m-1} & =\int\left(X_{1} \mu^{2(m-1)} w_{2(m-1)}-\overline{X_{1} \mu^{2(m-1)} w_{2(m-1)}}\right) d \theta . \\
\mu^{2 m} w_{2 m} & =\int\left(X_{1} \mu^{2 m-1} w_{2 m-1}\right) d \theta+\mu^{2 m} W_{2 m}(r) .
\end{aligned}
$$

Then the divergence $\operatorname{div} X$ of $X$ with respect to

$$
\omega=e^{w} d x d y
$$


is given by

$$
\operatorname{div} X=a-w_{0 r} b+\sum_{1 \leqslant m \leqslant n-1} \overline{X_{1} \mu^{2 m} w_{2 m}}+X_{1} \mu^{2 n} w_{2 n}
$$

(In the case $n=1$, read $\operatorname{div} X=a-w_{0 r} b+X_{1} \mu^{2} w_{2}$.)

Proof. Note that $\operatorname{div} X=X w-f_{x}$. Substituting $w=w_{0}+\mu w_{1}+\cdots$ $+\mu^{2 n} w_{2 n}$ in $X w-f_{x}$, and recalling that $X=-\partial / \partial \theta+X_{1}$, we obtain

$$
\begin{aligned}
\operatorname{div} X= & -\mu w_{1 \theta}+X_{1} w_{0}-f_{x} \\
& -\mu^{2} w_{2 \theta}+X_{1} \mu w_{1} \\
& -\cdots \\
& -\mu^{k} w_{k \theta}+X_{1} \mu^{k-1} w_{k-1} \\
& -\cdots \\
& -\mu^{2 n} w_{2 n \theta}+X_{1} \mu^{2 n-1} w_{2 n-1}+X_{1} \mu^{2 n} w_{2 n} .
\end{aligned}
$$

Since $X_{1} w_{0}=-\cos \theta f w_{0 r}$, our definition of $w_{1}$ implies that the 1st term becomes

$$
-\mu w_{1 \theta}+X_{1} w_{0}-f_{x}=a-w_{0 r} b .
$$

Note that $\mu w_{1}$ is odd (as a function of $\theta$ ), and hence that $X_{1} \mu w_{1}$ is also odd. Therefore the function $\mu^{2} w_{2}=\int\left(X_{1} \mu w_{1}\right) d \theta+\mu^{2} W_{2}(r)$ is well-defined and even. The 2nd term $-\mu^{2} w_{2 \theta}+X_{1} \mu w_{1}$ certainly vanishes. Generally, both $\mu^{k} w_{k}, X_{1} \mu^{k} w_{k}$ are even or odd functions of $\theta$ according as $k$ is even or odd. Our proposition is now obvious.

In order to apply this proposition to the van der Pol equation, let $f=\mu\left(x^{3}-x\right)$, and let $W_{0}(r)=-4 \log r$, and $W_{2}(r)$ a function to be determined. Define $w=w_{0}+\mu w_{1}+\mu^{2} w_{2}$ by the formulas in Proposition 2.4.2. We know already that

$$
\operatorname{div} X=a-w_{0 r} b+X_{1} \mu^{2} w_{2} .
$$

We shall prove that if $0<|\mu| \leqslant 1$, and if $W_{2}(r)$ is chosen suitably, then $\operatorname{div} X>$ positive constant or $<$ negative constant according as $\mu<0$ or $>0$. (See Proposition 2.4 .3 below. We will now give a heuristic argument for it.)

First, we contend that

$$
a-w_{0 r} b=-\mu
$$


In fact, using the formulas $\int_{0}^{2 \pi} \cos ^{2} \theta d \theta=\pi, \int_{0}^{2 \pi} \cos ^{4} \theta d \theta=\frac{3}{4} \pi$, we see that $a=-(\mu / 2)\left(3 r^{2}-2\right), b=(\mu / 2) r\left(\frac{3}{4} r^{2}-1\right)$. Our definition of $w_{0}$ implies that $a-w_{0 r} b=-\mu$.

Now, we must evaluate $X_{1} \mu^{2} w_{2}$. Note that $\mu w_{1}$ is odd (as a function of $\theta$ ), and so is $\mu w_{1 r}$. Hence $\mu w_{1 r}$ can be written in the form

$$
\mu w_{1 r}=\mu \sin \theta \Omega_{1},
$$

where $\Omega_{1}$ is even and in fact a function of $r, \cos \theta$. We consider $\Omega_{1}$ as a function of $r, x$. Recall that $\mu^{2} w_{2}=\int\left(X_{1} \mu w_{1}\right) d \theta+\mu^{2} W_{2}(r)$. Then we can write

$$
\mu^{2} w_{2}=\int(-r \sin \theta)\left(\frac{1}{r} \cos \theta f \mu \Omega_{1}-\frac{1}{r^{2}} f \mu w_{1 \theta}\right) d \theta+\mu^{2} W_{2}(r)
$$

Note that $\mu w_{1 \theta}=-f_{x}-\left(w_{0 r} / r\right) x f+\mu$. We now choose $W_{2}(r)$ so that $\mu^{2} w_{2}$ is divisible by $\left(x^{2}-1\right)^{2}$. In other words, we define

$$
\mu^{2} w_{2}=\int_{1}^{x}\left\{\frac{1}{r^{2}} x f \mu \Omega_{1}+\frac{1}{r^{2}} f\left(f_{x}+\frac{w_{0 r}}{r} x f-\mu\right)\right\} d x .
$$

Then $\mu^{2} w_{2 r}$ can be written in the form

$$
\mu^{2} w_{2 r}=\mu^{2}\left(x^{2}-1\right) \Omega_{2} .
$$

Thus

$$
\begin{aligned}
X_{1} \mu^{2} w_{2} & =-\cos \theta f \mu^{2} w_{2 r}+\frac{1}{r} \sin \theta f \mu^{2} w_{2 \theta} \\
& =f\left\{-\frac{1}{r} \mu^{2} x\left(x^{2}-1\right) \Omega_{2}-\sin ^{2} \theta \frac{f}{r^{2}}\left(\mu x \Omega_{1}+f_{x}+\frac{w_{0 r}}{r} x f-\mu\right)\right\} \\
& =-\frac{\mu f^{2}}{r^{2}}\left\{r \Omega_{2}+\sin ^{2} \theta\left(x \Omega_{1}+\frac{w_{0 r}}{\mu r} x f+\frac{1}{\mu} f_{x}-1\right)\right\} .
\end{aligned}
$$

It remains to determine $\Omega_{1}, \Omega_{2}$. By definition we have

$$
\mu w_{1 r}=-\int_{0}^{\theta}\left(f_{x}-\frac{4}{r} \cos \theta f-\mu\right)_{r} d \theta \text {. }
$$

Then it is easy to see that

$$
\mu w_{1 r}=2 \mu r \sin \theta \cos ^{3} \theta,
$$

whence 


$$
\Omega_{1}=\frac{2 x^{3}}{r^{2}}
$$

We contend that

$$
\Omega_{2}=\frac{1}{r^{5}}\left(-x^{6}+x^{4}+x^{2}+1\right)+\frac{1}{r^{3}}\left(2 x^{4}-\frac{1}{2} x^{2}-\frac{1}{2}\right) .
$$

Indeed, since $w_{0 r}=-4 / r$, the expression for $\mu^{2} w_{2}$ yields

$$
\mu^{2} w_{2 r}=\left\{\frac{1}{r^{4}} \int_{1}^{x}\left(2 \mu x^{4} f-4 x f^{2}\right) d x\right\}_{r}+\left\{\frac{1}{r^{2}} \int_{1}^{x} f\left(f_{x}-\mu\right) d x\right\}_{r} .
$$

Using the fact that

$$
\int_{1}^{x}\left(x^{2}-1\right) x^{3}\left(x^{2}-2\right) d x=\frac{1}{8}\left(x^{2}-1\right)^{2}\left(x^{4}-2 x^{2}-1\right),
$$

one can verify directly that

$$
\mu^{2} w_{2 r}=\mu^{2}\left(x^{2}-1\right)\left\{\frac{1}{r^{5}}\left(-x^{6}+x^{4}+x^{2}+1\right)+\frac{1}{r^{3}}\left(2 x^{4}-\frac{1}{2} x^{2}-\frac{1}{2}\right)\right\} .
$$

From this our contention follows. We now substitute these $\Omega_{1}, \Omega_{2}$ in the expression for $X_{1} \mu^{2} w_{2}$ obtained above. Omitting the intermediate calculations, we obtain

$$
\begin{aligned}
X_{1} \mu^{2} w_{2}=-\frac{\mu}{r^{2}} f^{2}\left\{\frac{1}{r^{4}}\left(x^{3}-\frac{3}{2} x\right)^{2}\right. & +\frac{5}{4 r^{4}}\left(r^{2}-x^{2}\right) \\
& \left.+\frac{1}{r^{4}}+\frac{7}{2 r^{2}}\left(x^{2}-\frac{1}{2}\right)+\sin ^{2} \theta\left(3 x^{2}-2\right)\right\} .
\end{aligned}
$$

We shall prove that

$$
-\frac{1}{\mu} X_{1} \mu^{2} w_{2} \geqslant(-1+\varepsilon) \mu^{2}
$$

on $R^{2}-\{(0,0)\}$, where $\varepsilon$ is a sufficiently small positive constant. This will prove that if $0<|\mu| \leqslant 1$, then

$$
\operatorname{div} X=-\mu\left(1-\frac{1}{\mu} X_{1} \mu^{2} w_{2}\right) \leqslant-\mu \varepsilon \quad \text { or } \geqslant-\mu \varepsilon
$$

according as $\mu>0$ or $\mu<0$.

In order to prove the estimate $(*)$, it suffices to prove that 
(i) $\frac{f^{2}}{r^{2}}\left\{\frac{1}{r^{4}}+\frac{7}{2 r^{2}}\left(x^{2}-\frac{1}{2}\right)\right\} \geqslant\left(-\frac{1}{2}+\varepsilon\right) \mu^{2}$.

(ii) $\frac{f^{2}}{r^{2}} \sin ^{2} \theta\left(3 x^{2}-2\right) \geqslant-\frac{1}{2} \mu^{2}$.

It is obvious that (i) holds on $x^{2} \geqslant \frac{1}{2}$. Moreover (i) holds on $r^{2} \leqslant \frac{4}{7}$. If $x^{2} \leqslant \frac{1}{2}$, and $r^{2} \geqslant 1$, we have

$$
\frac{f^{2}}{r^{2}}\left\{\frac{1}{r^{4}}+\frac{7}{2 r^{2}}\left(x^{2}-\frac{1}{2}\right)\right\} \geqslant \frac{f^{2}}{r^{2}}\left(-\frac{7}{4 r^{2}}\right) \geqslant-\frac{7}{4} f^{2} \geqslant-\frac{7}{27} \mu^{2},
$$

because

$$
\max _{x^{2} \leqslant 1} f^{2}=\frac{4}{27} \mu^{2}
$$

If $x^{2} \leqslant \frac{1}{2}$, and $\frac{4}{7} \leqslant r^{2} \leqslant 1$, then

$$
\begin{aligned}
\frac{f^{2}}{r^{2}}\left\{\frac{1}{r^{4}}+\frac{7}{2 r^{2}}\left(x^{2}-\frac{1}{2}\right)\right\} & =\frac{7 f^{2}}{2 r^{4}}\left(x^{2}-\frac{1}{2}+\frac{2}{7 r^{2}}\right) \\
& \geqslant \frac{7 f^{2}}{2 r^{4}}\left(-\frac{3}{14}\right) \geqslant-\frac{3}{4}\left(\frac{7}{4}\right)^{2} f^{2} \geqslant-\frac{49}{16 \cdot 9} \mu^{2} .
\end{aligned}
$$

These show (i). To prove (ii), note that $\cos ^{2} \theta \sin ^{2} \theta \leqslant \frac{1}{4}$. Since (ii) holds on $x^{2} \geqslant \frac{2}{3}$, it suffices to prove that (ii) holds on $x^{2} \leqslant \frac{2}{3}$. If $x^{2} \leqslant \frac{2}{3}$, we have

$$
\frac{f^{2}}{r^{2}} \sin ^{2} \theta\left(3 x^{2}-2\right)=\mu^{2} \cos ^{2} \theta \sin ^{2} \theta\left(x^{2}-1\right)^{2}\left(3 x^{2}-2\right) \geqslant-\frac{1}{2} \mu^{2} .
$$

The estimate (ii) is proved. Consequently, calculating the terms $w_{1}, w_{2}$ we obtain:

Proposition 2.4.3. Put

$$
\begin{aligned}
w= & -4 \log r+\frac{\mu}{r^{2}} x\left(x^{2}-2\right) y \\
& +\frac{\mu^{2}\left(x^{2}-1\right)^{2}}{2 r^{2}}\left\{x^{2}-\frac{1}{2}-\frac{1}{2 r^{2}}\left(x^{4}-2 x^{2}-1\right)\right\}, r^{2}=x^{2}+y^{2} .
\end{aligned}
$$

Then the divergence $\operatorname{div} X$ of

$$
X=\left(y-\mu\left(x^{3}-x\right)\right) \frac{\partial}{\partial x}-x \frac{\partial}{\partial y}
$$

with respect to $\omega=e^{w} d x d y$ satisfies the following inequality: There is a positive number $\varepsilon$ such that 


$$
\operatorname{div} X \begin{cases}\leqslant-\mu \varepsilon & \text { if } 0<\mu \leqslant 1, \\ \geqslant-\mu \varepsilon & \text { if }-1 \leqslant \mu<0 .\end{cases}
$$

Verifying that $\int_{R^{2}-D} \omega=\infty$ for $\mu \neq 0$, by Theorem 2.4.1 we have:

Corollary 2.4.4. Suppose that $0<|\mu| \leqslant 1$. Then system $(\mathrm{L})_{\mu\left(x^{3}-x\right)}$ has a unique closed integral curve, which is positively or negatively asymptotically stable according as $\mu>0$ or $\mu<0$. (Compare Lefschetz [9, p. 268], Hirsh-Smale [5, p. 218].)

\subsection{A proof of the Liénard's theorem}

Let $f_{1}(x)$ be a $C^{1}$ odd function such that $f_{1}(x)$ has a single positive zero $x_{1}, f_{1}<0$ on $\left(0, x_{1}\right)$, and such that $f_{1 x}(x)>0$ if $|x|>x_{1}$. Let $X=$ $\left(y-\mu f_{1}\right) \partial / \partial x-x \partial / \partial y$, where $\mu$ is a nonzero constant. We want to prove, by means of $\operatorname{div} X$, the following theorem.

Theorem 2.5.1 (cf. Lefschetz [9, p. 268]). The vector field $X$ possesses a unique closed integral curve. This integral curve is positively or negatively asymptotically stable according as $\mu>0$ or $\mu<0$.

Proof. Let $c, d:\left[-x_{1}, x_{1}\right] \rightarrow R$ be functions defined by

$$
\begin{aligned}
& c(x)=\max \left(\sqrt{x_{1}^{2}-x^{2}}, \mu f_{1}(x)\right), \\
& d(x)=\min \left(-\sqrt{x_{1}^{2}-x^{2}}, \mu f_{1}(x)\right) .
\end{aligned}
$$

We introduce a closed (topological) disk

$$
D=\left\{(x, y)|| x \mid \leqslant x_{1}, d(x) \leqslant y \leqslant c(x)\right\} .
$$

It is easily verified that $D$ is positively or negatively invariant for $X$ according as $\mu<0$ or $\mu>0$. Moreover, noting that $X\left(x^{2}+y^{2}\right)=-2 \mu x f_{1}$, one sees easily that $D$ contains no closed integral curve. On the other hand, using the same Liapunov function as in Graef [4, p. 45], we see that there is a closed disk $E$ containing $D$ such that $E$ is positively or negatively invariant for $X$ according as $\mu>0$ or $\mu<0$. Hence $E-D$ contains a closed integral curve (Poincaré's closed path theorem). We shall prove its uniqueness. We define $w: R^{2}-D \rightarrow R$ by the following formula:

$$
e^{w(x, y)}=\left\{\begin{array}{cl}
\frac{y}{y-\mu f_{1}} & \text { if }|x| \leqslant x_{1} \\
1 & \text { if }|x| \geqslant x_{1}
\end{array}\right.
$$


The definition of $D$ assures us that $w$ is well-defined. It is obvious that $w$ is continuous (on $R^{2}-D$ ) and of class $C^{1}$ except on $|x|=x_{1}$. Now, we let $\omega=e^{w} d x d y$ and consider the divergence $\operatorname{div} X$ (defined on $R^{2}-D-$ $\left.\left\{|x|=x_{1}\right\}\right)$ of $X$ with respect to $\omega$. Using the formula $\operatorname{div} X=X w-\mu f_{1 x}$, we obtain

$$
\operatorname{div} X=\left\{\begin{array}{cc}
\frac{\mu x f_{1}}{y\left(y-\mu f_{1}\right)} & \text { on }|x|<x_{1}, \\
-\mu f_{1 x} & \text { on }|x|>x_{1} .
\end{array}\right.
$$

Hence

$$
\frac{1}{\mu} \operatorname{div} X \leqslant 0
$$

on $\left\{(x, y) \in R^{2}-D \mid x \neq 0, x \neq \pm x_{1}\right\}$ and the equality holds only on $x=0$. Noting that $X \mid R^{2}-D$ traverses the lines $x=0$ and $|x|=x_{1}(y \neq 0)$, we conclude that if $\mu>0$ (resp. $<0$ ), any closed integral curve in $R^{2}-D$ is positively (resp. negatively) asymptotically stable. Hence the closed integral curve is unique (cf. [9, p. 235]). Theorem 2.5.1 is proved.

\section{§3. Proof of Theorem 1.1.2}

As in $\S 1$, let $M$ be a connected, closed 2-dimensional $C^{3}$ manifold with a fixed area-element $\omega$ (of class $C^{2}$ ).

3.1. Geometric conditions for $X$ and a decomposition of $M$

Let $X$ be a $C^{1}$ vector field on $M$. We consider the following conditions:

(a) Each singular point of $X$ is either a source, a sink or a saddle point (cf. [5]).

(b) For any singular point $p$ of $X$, we have

$$
(\operatorname{div} X)(p) \neq 0 \text {. }
$$

(c) For any periodic trajectory $\gamma(t)$ of $X$ with period $\tau$, we have

$$
\int_{0}^{\tau}(\operatorname{div} X)(\gamma(t)) d t \neq 0
$$

(d) If there is a trajectory $\gamma(t)$ which connects two saddle points

$$
\lim _{t \rightarrow-\infty} \gamma(t)=p, \quad \lim _{t \rightarrow+\infty} \gamma(t)=q,
$$


and if $(\operatorname{div} X)(p)$ is negative, then $(\operatorname{div} X)(q)$ is also negative.

Remark 1. Let $X$ satisfy condition (b). Then, for any $C^{1}$ function $v: M \rightarrow R$, the vector field $Y=e^{v} X$, also, satisfies condition (b) because of the formula $\operatorname{div} Y=X\left(e^{v}\right)+e^{v} \operatorname{div} X$ and the trivial fact that $X\left(e^{v}\right)(p)=0$ for any singular point $p$ of $X$.

Remark 2. For a singular point $p$ of $X$, if $(\operatorname{div} X)(p)>0($ resp. $<0)$, then a function $v: M \rightarrow R$ satisfying

$$
e^{v(p)}=\frac{1}{(\operatorname{div} X)(p)}\left(\operatorname{resp} \cdot \frac{-1}{(\operatorname{div} X)(p)}\right)
$$

has the following property:

$$
\left(\operatorname{div} e^{v} X\right)(p)=e^{v(p)}(\operatorname{div} X)(p)=1(\text { resp. }-1) .
$$

In order to describe the qualitative property of $X$ with these conditions, we need the definition of pathpolygon.

Definition (cf. Lefschetz [9]). A pathpolygon $\Gamma$ is a closed subset of $M$ having the following properties:

(i) The set $\Gamma$ is written as a union

$$
\Gamma=p_{1} \cup\left\{\gamma_{1}\right\} \cup p_{2} \cup\left\{\gamma_{2}\right\} \cup \cdots \cup p_{r} \cup\left\{\gamma_{r}\right\}
$$

where $p_{i}$ are saddle points (not necessarily distinct), and $\gamma_{i}$ are trajectories with $\lim _{t \rightarrow-\infty} \gamma_{i}(t)=p_{i}, \lim _{t \rightarrow+\infty} \gamma_{i}(t)=p_{i+1}\left(p_{r+1}=p_{1}\right)$, and orbits $\left\{\gamma_{i}\right\}=$ $\bigcup_{-\infty<t<\infty} \gamma_{i}(t)$.

(ii) There is a continuous map $h: S^{1} \times[0,1) \rightarrow M$ such that the image $h\left(S^{1} \times 0\right)$ coincides with $\Gamma$ and such that the restriction $h \mid: S^{1} \times(0,1) \rightarrow$ $h\left(S^{1} \times(0,1)\right)$ is a homeomorphism whose image intersects with neither local stable manifolds nor local unstable manifolds of the saddle points $p_{i}$.

The set $h\left(S^{1} \times[0,1)\right)$ will be called a collar of the pathpolygon $\Gamma$. An $\alpha$-limit pathpolygon is defined to be a pathpolygon $\Gamma$ which has a collar $W$ such that the $\alpha$-limit set of any point in $W-\Gamma$ coincide with $\Gamma$. Similarly, we can define an $\omega$-limit pathpolygon.

Lemma 3.1.1. Suppose that $X$ satisfies conditions (b), (d), and has a pathpolygon $\Gamma$. Let $p$ be a saddle point in $\Gamma$. If $(\operatorname{div} X)(p)$ is positive, then $\Gamma$ is an $\alpha$-limit pathpolygon. If $(\operatorname{div} X)(p)$ is negative, then $\Gamma$ is an $\omega$-limit pathpolygon. 
Proof. Let $\Gamma=p_{1} \cup\left\{\gamma_{1}\right\} \cup p_{2} \cup\left\{\gamma_{2}\right\} \cdots \cup p_{r} \cup\left\{\gamma_{r}\right\}$ as in the definition, and let $W$ be a collar of $\Gamma$. Replacing $X$ by $-X$ if necessary, we may assume that $(\operatorname{div} X)\left(p_{1}\right)<0$. We shall prove that $\Gamma$ is an $\omega$-limit pathpolygon. Note that condition (d) implies that $(\operatorname{div} X)\left(p_{i}\right)<0$ for all $i$. Let $q \in \Gamma$ be a regular point. Choose a local coordinate system $(t, r)$ in a coordinate neighborhood $U$ of $q$ such that $X=\partial / \partial t$ on $U, \omega= \pm d t d r$ on the segment $t=0$, and such that $U \cap \Gamma=\{(t, r) \mid r=0\}, U \cap W=\{(t, r) \mid r \geqslant 0\}$. By means of the normal $\{(t, r) \mid t=0, r \geqslant 0\}$ with coordinate function $r$, we can define a so-called Poincaré map $\rho:[0, \varepsilon) \rightarrow[0,1)$ for a sufficiently small positive number $\varepsilon$. Let $U_{i}$ be neighborhoods of $p_{i}$ such that $(\operatorname{div} X)(x)<$ $-\delta$ for $x \in U_{i}$, where $\delta$ is a positive constant. Note that there exists a positive constant $l$ satisfying the following condition. For any $T>0$, there exists $r_{0}>0$ such that for any point $p$ with coordinates $(0, r), 0<$ $r<r_{0}$, the trajectory $\gamma(t)$ starting from $p$ has the following properties:

(i) The trajectory $\gamma(t)$ crosses the normal $\{(0, r) \mid r \geqslant 0\}$ at the point with coordinates $(0, \rho(r))$ after a time $\tau>T$ for the first time.

(ii) The point $\gamma(t)$ is contained in $\bigcup_{i=1}^{r} U_{i}$ for any $t \in[0, \tau]-S$, where $S$ is a union of a finite number of subintervals of $[0, \tau]$ such that the sum of the lengths is less than $l$.

Using this fact and the formula

$$
\frac{d \rho(r)}{d r}=\exp \left(\int_{0}^{\tau}(\operatorname{div} X)\left(\phi_{t}(p)\right) d t\right)
$$

( $\left\{\phi_{t}\right\}$ denotes the 1-parameter transformation group generated by $X$ ), we can easily see that $d \rho(r) / d r \rightarrow+0$ as $r \rightarrow+0$, and hence that $\rho(r)<r$ for sufficiently small $r>0$. This shows that $\Gamma$ is an $\omega$-limit pathpolygon. Our lemma is proved.

Now, let $X$ be a vector field satisfying conditions (a)-(d). We introduce the following subsets of $M$. Let $\Omega$ be the set consisting of singular points and of limit cycles of $X$. Let $\Omega_{+}$be the subset of $\Omega$ consisting of singular points at which $\operatorname{div} X$ is positive, and of $\alpha$-limit cycles. Similarly, let $\Omega_{-}$be the subset of $\Omega$ consisting of singular points at which $\operatorname{div} X$ is negative, and of $\omega$-limit cycles. It is clear that $\Omega=\Omega_{+} \cup \Omega_{-}$ (disjoint union) because of conditions (b), (c). Furthermore, we denote by $\Omega_{+}^{\times}, \Omega_{-}^{\times}$the sets consisting of saddle points at which $\operatorname{div} X$ is positive, negative respectively. We put

$$
\Xi_{+}=\left\{p \in M \mid p \notin \Omega_{+}^{\times}, \omega-\lim (p) \in \Omega_{+}^{\times}\right\},
$$




$$
\begin{aligned}
E_{-} & =\left\{p \in M \mid p \notin \Omega_{-}^{\times}, \alpha-\lim (p) \in \Omega_{-}^{\times}\right\}, \\
\Xi & =\Xi_{+} \cup \Xi_{-},
\end{aligned}
$$

where $\alpha$-lim $(p), \omega$-lim $(p)$ denote the $\alpha$-limit set of $p, \omega$-limit set of $p$ respectively (cf. [5]). Note that $\Xi_{+} \cap \Xi_{-}=\phi$ because of condition (d). It is obvious that the sets introduced above are invariant sets of $X$ (in the both directions).

Proposition 3.1.2. Suppose that $X$ satisfies conditions (a)-(d) and the following condition:

(e) The $\alpha$ and $\omega$-limit sets of every trajectory are either singular points, closed orbits or pathpolygons.

Let $\Pi_{+}, \Pi_{-}$denote the unions of $\alpha$-limit pathpolygons, $\omega$-limit pathpolygons respectively. Then $M$ is expressed as the disjoint union

$$
M=\Omega \cup \Xi \cup M_{0},
$$

where

$$
M_{0}=\left\{p \in M \mid \alpha-\operatorname{limit}(p) \subset \Omega_{+} \cup \Pi_{+}, \omega-\operatorname{limit}(p) \subset \Omega_{-} \cup \Pi_{-}\right\} .
$$

The subset $M_{0}$ is open, and the subset $\Omega \cup \Xi$ is closed. The set $\Omega$ consists of a finite number of singular points and a finite number of closed orbits. Furthermore, we have

$$
\begin{aligned}
& \Omega_{+} \cup \Xi_{+}=\left\{p \in M \mid \alpha-\lim (p) \subset \Omega_{+} \cup \Pi_{+}, \omega-\lim (p) \subset \Omega_{+}\right\} \\
& \Omega_{-} \cup \Xi_{-}=\left\{p \in M \mid \alpha-\lim (p) \subset \Omega_{-}, \omega-\lim (p) \subset \Omega_{-} \cup \Pi_{-}\right\} .
\end{aligned}
$$

Proof. It is obvious that, $\Omega, \Xi, M_{0}$ are disjoint each other. By condition (e) we see that for any point $p$ of $M$, if $p \notin \Omega$ then the $\alpha$-limit set of $p$ coincides with a source, saddle point, $\alpha$-limit cycle or $\alpha$-limit pathpolygon, and the $\omega$-limit set of $p$ coincides with a sink, saddle point, $\omega$ limit cycle or $\omega$-limit pathpolygon. In other words, $M$ is expressed as a disjoint union

$$
M=\Omega \cup\left\{p \in M-\Omega \mid \begin{array}{l}
\alpha-\lim (p) \subset \Omega_{+} \cup \Omega_{-}^{\times} \cup \Pi_{+} \\
\omega-\lim (p) \subset \Omega_{-} \cup \Omega_{+}^{\times} \cup \Pi_{-}
\end{array}\right\} .
$$

Note that condition (d) implies that

$$
\left\{p \in M \mid \alpha-\lim (p) \in \Omega_{-}^{\times}, \omega-\lim (p) \in \Omega_{+}^{\times}\right\}=\phi .
$$

From these facts and the definitions of $\Xi_{ \pm}$, it follows immediately that $M=\Omega \cup \Xi_{+} \cup E_{-} \cup M_{0}$ and hence $M=\Omega \cup \Xi \cup M_{0}$. It is obvious that 
$M_{0}$ is open and hence $\Omega \cup \Xi$ is closed. The former part of our assertion is proved. We see easily that

$$
\begin{aligned}
& \Omega_{+} \cup \Xi_{+} \subset\left\{p \in M \mid \alpha-\lim (p) \subset \Omega_{+} \cup \Pi_{+}, \omega-\lim (p) \subset \Omega_{+}\right\}, \\
& \Omega_{-} \cup \Xi_{-} \subset\left\{p \in M \mid \alpha-\lim (p) \subset \Omega_{-}, \omega-\lim (p) \subset \Omega_{-} \cup \Pi_{-}\right\} .
\end{aligned}
$$

Since $M_{0}$ and the two sets of the right-hand sides of these inclusion relations are disjoint each other, and since $M=\Omega \cup \Xi \cup M_{0}$ (disjoint union) as proved above, we conclude that in the inclusion relations, the equalities hold.

It remains to prove that $\Omega$ consists of a finite number of singular points and a finite number of closed orbits. To prove this, it suffices to prove that the number of closed orbits is finite. Lemma 3.1.1 implies that each pathpolygon (if any) has a collar containing no closed orbit. Therefore in order to prove the finiteness of the number of closed orbits, it suffices to prove the following lemma.

Lemma 3.1.3. Suppose that a $C^{1}$ vector field $X$ satisfies conditions (a), (c), (e), and has an infinite number of closed orbits. Then there exists a pathpolygon $\Gamma$ such that any collar of $\Gamma$ contains an infinite number of closed orbits.

Proof. Let $\pi_{i}, i \in I$, be the infinite number of closed orbits. Denote by $\left\{\pi_{i}\right\}^{\prime}$ the set of points $p$ such that the set $\left\{i \in I \mid \pi_{i} \cap U \neq \phi\right\}$ is infinite for any neighborhood $U$ of $p$. Then, using conditions (a), (c), (e) we see that

(i) If $p \in\left\{\pi_{i}\right\}^{\prime}$, then $\omega$-lim $(p)$ is a saddle point, and $\omega-\lim (p) \in\left\{\pi_{i}\right\}^{\prime}$.

(ii) If $p$ is a saddle point and $p \in\left\{\pi_{i}\right\}^{\prime}$, then there is a trajectory $\gamma(t)$ such that $\lim _{t \rightarrow-\infty} \gamma(t)=p$, and $\gamma(t) \in\left\{\pi_{i}\right\}^{\prime}$ for each $t$.

Now, since $M$ is compact, there is a point $p \in\left\{\pi_{i}\right\}^{\prime}$. Then, from (i) it follows that $\omega-\lim (p)$ is a saddle point $p_{1} \in\left\{\pi_{i}\right\}^{\prime}$. By (ii) we have a trajectory $\gamma_{1}(t)$ such that $\lim _{t \rightarrow-\infty} \gamma_{1}(t)=p_{1}$, and $\gamma_{1}(t) \in\left\{\pi_{i}\right\}^{\prime}$ for each $t$. Again from (i) it follows that $\omega$-lim $\left(\gamma_{1}(t)\right)$ is a saddle point $p_{2} \in\left\{\pi_{i}\right\}^{\prime}$, and hence $\lim _{t \rightarrow \infty} \gamma_{1}(t)$ $=p_{2}$. Repeating the same arguments, since the number of saddle points is finite, we obtain a pathpolygon $\Gamma$. It can be directly checked that $\Gamma$ has the desired property. This completes the proof of our proposition. 
3.2. Elementary vector fields

Let $Y$ be a continuous vector field on $M$. Assume that $Y$ generates a unique 1-parameter transformation group $\left\{\psi_{s}\right\}$ of $M$ and that $Y$ has a divergence $\operatorname{div} Y$. Furthermore, we assume that the divergence $\operatorname{div} Y$ is continuous, and the derivative $Y(\operatorname{div} Y$ ) exists and is continuous (on $M$ ). Here, we define $\operatorname{div} Y$ to be a function on $M$ such that for any $s \in R$ and any open set $U \subset M$, the area of the set $\psi_{s}(U)$ is given by

$$
\int_{x \in U}\left\{\exp \int_{0}^{s}(\operatorname{div} Y)\left(\psi_{s}(x)\right) d s\right\} \omega .
$$

We define the derivative $Y f$ of a function $f$ by the formula

$$
(Y f)(x)=\lim _{s \rightarrow 0} \frac{1}{s}\left\{f\left(\psi_{s}(x)\right)-f(x)\right\} .
$$

Definition. Let $\lambda$ be a positive number. We shall say that the vector field $Y$ is elementary with stability exponent $\lambda$ if $Y$ satisfies the equation

$$
(Y-\operatorname{div} Y) \operatorname{div} Y=-\lambda^{2},
$$

i.e.,

$$
(Y(\operatorname{div} Y))(p)-(\operatorname{div} Y)^{2}(p)=-\lambda^{2}
$$

for all $p \in M$.

Definition. Let $X$ be a vector field on $M$ (such that $X$ generates a unique 1-parameter transformation group). A function $u: M \rightarrow R$ is said to be of class $C^{r}$ with respect to $X$ if $u$ is continuous and if the derivatives $X u, X^{2} u=X(X u), \cdots, X^{r} u$ exist and are continuous on $M$.

Lemma 3.2.1. Let $X$ be a $C^{2}$ vector field, and let $\left\{\phi_{t}\right\}$ be the 1-parameter transformation group generated by $X$. Let $u: M \rightarrow R$ be a function of class $C^{2}$ with respect to $X$. Then the vector field $Y=e^{u} X$ generates $a$ unique 1-parameter transformation group $\left\{\psi_{s}\right\}$ and has a divergence $\operatorname{div} Y$ of class $C^{1}$ with respect to $Y$. The divergence $\operatorname{div} Y$ is given by

$$
\operatorname{div} Y=X\left(e^{u}\right)+e^{u}(\operatorname{div} X) .
$$

Furthermore, for each point $p$ in $M$, there is a function $S(t)$ such that, for all $t$,

$$
\begin{aligned}
& \text { (i ) } \phi_{t}(p)=\psi_{S(t)}(p) . \\
& \text { (ii) } \frac{d S(t)}{d t}=e^{-u\left(\phi_{t}(p)\right)}, S(0)=0 .
\end{aligned}
$$


where

(iii) $\int_{0}^{t} \delta(t) d t=e^{u(p)} \int_{0}^{S(t)} \varepsilon(s) d s$

$$
\delta(t)=\exp \left\{\int_{0}^{t}(\operatorname{div} X)\left(\phi_{t}(p)\right) d t\right\}, \quad \varepsilon(s)=\exp \left\{\int_{0}^{s}(\operatorname{div} Y)\left(\psi_{s}(p)\right) d s\right\} .
$$

Proof. For $p \in M$, let $S(t, p)$ be the solution of the differential equation

$$
\frac{d S(t, p)}{d t}=e^{-u\left(\phi_{t}(p)\right)} \quad \text { with } S(0, p)=0 .
$$

We define $\psi_{s}$ by the formula

$$
\psi_{s}(p)=\phi_{t}(p)
$$

where $s=S(t, p)$. We can easily verify that $\left\{\psi_{s}\right\}$ is the unique 1-parameter transformation group generated by the vector field $Y$. To prove $\operatorname{div} Y=X\left(e^{u}\right)+e^{u}(\operatorname{div} X)$, it suffices to prove that this formula holds in a flow box at any regular point of $X$. This can be directly verified. It remains to prove (iii). For this, we put

$$
h(t)=\int_{0}^{t} \delta(t) d t-e^{u(p)} \int_{0}^{s(t)} \varepsilon(s) d s .
$$

We shall prove $h(t) \equiv 0$. First, note that $h(0)=0$. From the formulas in (ii) it follows immediately that

$$
\frac{d h(t)}{d t}=\delta(t)-e^{u(p)-u\left(\phi_{t}(p)\right)} \varepsilon(S(t)), \quad h^{\prime}(0)=0 .
$$

Furthermore, using the formulas

$$
\begin{gathered}
\frac{d \delta(t)}{d t}=\delta(t) \cdot(\operatorname{div} X)\left(\phi_{t}(p)\right), \\
\frac{d \varepsilon(S(t))}{d t}=\varepsilon(S(t)) \cdot(\operatorname{div} Y)\left(\phi_{t}(p)\right) \cdot e^{-u\left(\phi_{t}(p)\right)}, \\
(\operatorname{div} Y)\left(\phi_{t}(p)\right)=e^{u\left(\phi_{t}(p)\right)} \cdot\left\{\frac{d u\left(\phi_{t}(p)\right)}{d t}+(\operatorname{div} X)\left(\phi_{t}(p)\right)\right\},
\end{gathered}
$$

we obtain

$$
\frac{d^{2} h(t)}{d t^{2}}=\frac{d h(t)}{d t} \cdot(\operatorname{div} X)\left(\phi_{t}(p)\right)
$$

Since $h(0)=h^{\prime}(0)=0$, by uniqueness theorem of differential equations we 
conclude that $h(t) \equiv 0$, as desired. This completes the proof of our lemma.

Lemma 3.2.2. Let $X$ be a $C^{2}$ vector field. Let $u: M \rightarrow R$ be a function of class $C^{2}$ with respect to $X$. Let $\lambda$ be a positive number. Then, in order that $e^{u} X$ is elementary with stability exponent $\lambda$ it is necessary and sufficient that the identity

$$
(X-\operatorname{div} X) X u+(X-\operatorname{div} X) \operatorname{div} X+\lambda^{2} e^{-2 u}=0
$$

holds.

Proof. By Lemma 3.2.1 we have $\operatorname{div}\left(e^{u} X\right)=e^{u}(X u+\operatorname{div} X)$. By definition, $e^{u} X$ is elementary with stability exponent $\lambda$ if and only if $\left(e^{u} X\right.$ $\left.-\operatorname{div}\left(e^{u} X\right)\right)\left(\operatorname{div} e^{u} X\right)=-\lambda^{2}$. It is easy to see that this equation can be written

$$
e^{2 u}\{(X-\operatorname{div} X) X u+(X-\operatorname{div} X) \operatorname{div} X\}=-\lambda^{2} .
$$

This proves our lemma.

From this lemma we get immediately:

Lemma 3.2.3. Let $X, u$ be as in the previous lemma. Let $u_{0}$ be a real number. Then $e^{u} X$ is elementary with stability exponent 1 if and only if $e^{u+u_{0}} X$ is elementary with stability exponent $e^{u_{0}}$.

The analytic property of elementary vector fields is given by the following lemma.

Lemma 3.2.4. Let $Y$ be an elementary field with stability exponent 1. Let $\left\{\psi_{s}\right\}$ be the 1-parameter transformation group generated by $Y$. Let $p \in M$, and put

$$
\varepsilon(s)=\exp \left\{\int_{0}^{s}(\operatorname{div} Y)\left(\psi_{s}(p)\right) d s\right\}
$$

Then we have

$$
\varepsilon(s)=\frac{1}{c e^{s}+(1-c) e^{-s}}
$$

where $c$ is a constant satisfying $0 \leqslant c \leqslant 1$. Furthermore, two infinite integrals $\int_{0}^{\infty} \varepsilon(s) d s, \int_{-\infty}^{0} \varepsilon(s) d s$ converge and coincide if and only if $(\operatorname{div} Y)(p)$ $=0$. If that is the case, we have 


$$
\varepsilon(s)=\frac{1}{\cosh s}, \quad(\operatorname{div} Y)\left(\psi_{s}(p)\right)=-\tanh s
$$

Proof. Since $\varepsilon(s)$ satisfies the differential equation

$$
\frac{d \varepsilon(s)}{d s}=(\operatorname{div} Y)\left(\psi_{s}(p)\right) \cdot \varepsilon(s)
$$

we see that the function $1 / \varepsilon(s)$ satisfies differential equations

$$
\begin{gathered}
\frac{d}{d s}\left(\frac{1}{\varepsilon(s)}\right)=-(\operatorname{div} Y)\left(\psi_{s}(p)\right) \cdot \frac{1}{\varepsilon(s)}, \\
\frac{d^{2}}{d s^{2}}\left(\frac{1}{\varepsilon(s)}\right)=\left((\operatorname{div} Y)^{2}-Y(\operatorname{div} Y)\right)\left(\psi_{s}(p)\right) \cdot \frac{1}{\varepsilon(s)} .
\end{gathered}
$$

From the assumption $(Y-\operatorname{div} Y) \operatorname{div} Y=-1$, we obtain

$$
\frac{d^{2}}{d s^{2}}\left(\frac{1}{\varepsilon(s)}\right)=\frac{1}{\varepsilon(s)}
$$

which is a linear differential equation with constant coefficients. Since $\varepsilon(0)=1$, we conclude that

$$
\frac{1}{\varepsilon(s)}=c e^{s}+(1-c) e^{-s}
$$

where $c$ is a constant. Since $\varepsilon(s)>0$, the constant $c$ satisfies $0 \leqslant c \leqslant 1$. If $c=0$ (or 1 ), it is obvious that $\int_{0}^{\infty} \varepsilon(s) d s=\infty$ (or $\left.\int_{-\infty}^{0} \varepsilon(s) d s=\infty\right)$. If $0<$ $c<1$, it is obvious that $\int_{0}^{\infty} \varepsilon(s) d s, \int_{-\infty}^{0} \varepsilon(s) d s$ converge, and that $\int_{0}^{\infty} \varepsilon(s) d s=$ $\int_{-\infty}^{0} \varepsilon(s) d s$ if and only if $c=\frac{1}{2}$. On the other hand, since

$$
(\operatorname{div} Y)\left(\psi_{s}(p)\right)=\frac{1}{\varepsilon(s)} \frac{d \varepsilon(s)}{d s}=-\frac{c e^{s}-(1-c) e^{-s}}{c e^{s}+(1-c) e^{-s}}
$$

we see that $(\operatorname{div} Y)(p)=0$ if and only if $c=\frac{1}{2}$. Consequently we conclude that $\int_{0}^{\infty} \varepsilon(s) d s=\int_{-\infty}^{0} \varepsilon(s) d s$ if and only if $(\operatorname{div} Y)(p)=0$. The latter part of our assertion is obvious, because if $c=\frac{1}{2}$, then $\varepsilon(s)=1 / \cosh s,(\operatorname{div} Y)\left(\psi_{s}(p)\right)$ $=-\tanh s$.

Remark. In order to explain the analytic property of elementary vector fields, let us recall the meaning of $\varepsilon(s)=\exp \left\{\int_{0}^{s}(\operatorname{div} Y)\left(\psi_{s}(p)\right) d s\right\}$. 
For simplicity, we assume that $Y$ is of class $C^{2}$. Then the 1-parameter transformation group $\left\{\psi_{s}\right\}$ induces the 1-parameter transformation group $\left\{\psi_{s *}\right\}$ on the tangent bundle of $M$. Let $p \in M$, and let $\left\{v_{1}, v_{2}\right\}$ be a basis of the tangent space at $p$. Denote by $\left|\left(v_{1}, v_{2}\right)\right|$ the area of the parallelogram with the two sides $v_{1}, v_{2}$. Then it is an elementary fact that the relation between $\left|\left(v_{1}, v_{2}\right)\right|$ and $\left|\left(\psi_{s *} v_{1}, \psi_{s *} v_{2}\right)\right|$ is given by the formula

$$
\left|\left(\psi_{s *} v_{1}, \psi_{s *} v_{2}\right)\right|=\left|\left(v_{1}, v_{2}\right)\right| \exp \left\{\int_{0}^{s}(\operatorname{div} Y)\left(\psi_{s}(p)\right) d s\right\}
$$

Therefore, if $Y$ is elementary with stability exponent 1 , then the function

$$
\frac{1}{\left|\left(\psi_{s *} v_{1}, \psi_{s *} v_{2}\right)\right|}
$$

of $s$ is of the form const. $\left\{c e^{s}+(1-c) e^{-s}\right\}$, which is one of the simplest functions in analysis.

The preceding lemma implies the following geometric property of elementary vector fields.

Proposition 3.2.5. Let $Y$ be an elementary vector field with stability exponent 1. Let $\left\{\psi_{s}\right\}$ be the 1-parameter transformation group generated by Y. Put

$$
\begin{aligned}
& N_{ \pm}=\{p \in M \mid(\operatorname{div} Y)(p)= \pm 1\} \\
& M_{0}=\{p \in M||(\operatorname{div} Y)(p) \mid<1\}
\end{aligned}
$$

Then we have

$$
M=N_{+} \cup N_{-} \cup M_{0} \text { (disjoint union) . }
$$

The two sets $N_{+}, N_{-}$are closed and invariant by $\left\{\psi_{s}\right\}$. Furthermore, for any point $p$ in $M_{0}$, the $\alpha$-limit set of $p$ is contained in $N_{+}$and the $\omega$-limit set of $p$ is contained in $N_{-}$.

Considering the function $f(s)=(1 / \varepsilon(s))(d \varepsilon(s) / d s)$ instead of $\varepsilon(s)$, we see that the proof of Lemma 3.2.4 implies the following:

LEMMA 3.2.6. Every bounded solution $f=f(s)$ of a differential equation

$$
\frac{d f}{d s}-f^{2}+1=0
$$

is given by 


$$
f(s)=-\frac{c e^{s}-(1-c) e^{-s}}{c e^{s}+(1-c) e^{-s}}
$$

where $c$ is an arbitrary constant satisfying $0 \leqslant c \leqslant 1$. Hence, the solution is constant if and only if $c=0$ or $c=1$. If $0<c<1$, then the solution $f$ has the following properties:

$$
\begin{gathered}
-1<f(s)<1 \quad \text { for all } s \in R, \\
\lim _{s \rightarrow \pm \infty} f(s)=\mp 1 .
\end{gathered}
$$

ExAmple. Let $R^{2}$ be a plane with coordinate system $(x, y)$. Let $T^{2}$ be the torus defined by $R^{2} /(2 \pi Z \times 2 \pi Z)$, and let $\omega=d x d y$ be the areaelement on $T^{2}$. Let $Y$ be a vector field

$$
Y=f(x) \frac{\partial}{\partial x}+g(x) \frac{\partial}{\partial y},
$$

and assume that $g(x)$ is continuous and periodic with period $2 \pi$. It is directly verified that if $f(x)=\lambda / m \sin m x$ ( $\lambda$ is a positive number and $m$ is a positive integer), then $Y$ is an elementary vector field on $T^{2}$ with stability exponent $\lambda$. More generally, using the fact that a function $f$ defined by

$$
f(x)= \begin{cases}\frac{1}{\mu} \sin \mu x & \text { for } x \geqslant 0 \\ \frac{1}{\nu} \sin \nu x & \text { for } x \leqslant 0(\mu, \nu ; \text { constants })\end{cases}
$$

is of class $C^{2}$ (at $x=0$ ), we get many examples of nonsingular elementary vector fields on $T^{2}$. Conversely, we can prove that if $X$ is a nonsingular vector field on $T^{2}$ and if $X$ is structurally stable, then there is a homeomorphism which preserves the area-element $\omega$ and by which $X$ is equivalent to the vector field mentioned above. Indeed, in the proof of Proposition 3.3.2, we seek out essentially such a homeomorphism.

3.3. Existence theorems of the multiplier $e^{u}$ such that $e^{u} X$ is elementary

In 3.2, we introduced a class of vector fields (elementary vector fields). Despite its rather simple definition, this class is of generality in the following sense. For almost every vector field $X$, by choosing a suitable multiplier, we can make $X$ elementary. More precisely, as a (slightly 
generalized) reformulation of Theorem 1.1.2, we have:

Theorem 3.3.1. Let $X$ be a $C^{2}$ vector field on $M$. Assume that $X$ satisfies conditions (a)-(e) as in Proposition 3.1.2. Then there exists a function $u: M \rightarrow R$ and a positive number $\lambda$ such that

(i) The function $u$ is of class $C^{2}$ with respect to $X$.

(ii) The vector field $e^{u} X$ is elementary with stability exponent $\lambda$.

(iii) The integral of $u$ on $M, \int_{M} u \omega$, is equal to zero.

Furthermore, these $u$ and $\lambda$ are uniquely determined.

This theorem is a direct consequence of Lemma 3.2.3 and the following proposition.

Proposition 3.3.2. Let $X$ be the same as in the preceding theorem. Then there exists uniquely a function $u: M \rightarrow R$ such that

(i) $u$ is of class $C^{2}$ with respect to $X$.

(ii) $e^{u} X$ is elementary with stability exponent 1 .

This proposition will be proved in 3.6. In 3.4, 3.5 we shall prepare for the proof.

3.4. Solving linear differential equations with boundary conditions

We give some elementary facts of 1st order linear differential equations. Let $d: \boldsymbol{R} \rightarrow \boldsymbol{R}$ be a continuous function. Put

$$
\delta(t)=\exp \left\{\int_{0}^{t} d(t) d t\right\}
$$

LEMma 3.4.1. Let $d=d(t)$ be periodic with period $\tau$. Assume that

$$
\delta(\tau)=\exp \left\{\int_{0}^{\tau} d(t)\right\} \neq 1
$$

Then the solution of a differential equation

$$
\frac{d g(t)}{d t}+d(t) g(t)=1(\text { resp. }-1)
$$

with condition

$$
g(0)=g(\tau)
$$

is given by 


$$
g(t)=\frac{\int_{0}^{\tau} \delta(s+t) d s}{(\delta(\tau)-1) \delta(t)}\left(\operatorname{resp} \cdot \frac{\int_{0}^{\tau} \delta(s+t) d s}{(1-\delta(\tau)) \delta(t)}\right)
$$

Proof. The general solution of $d g / d t+d \cdot g=1$ is given by

$$
g(t)=\left\{c+\int_{0}^{t} \delta(t) d t\right\} / \delta(t) .
$$

From the condition $g(0)=g(\tau)$, we get

$$
c=\int_{0}^{\tau} \delta(t) d t /(\delta(\tau)-1) .
$$

On the other hand, by change of variable we have

$$
\int_{0}^{\tau} \delta(s+t) d s=\int_{0}^{\tau} \delta(t) d t+\int_{0}^{t} \delta(\tau+t) d t-\int_{0}^{t} \delta(t) d t
$$

Note that $\delta(\tau+t)=\delta(\tau) \delta(t)$ because of the periodicity of $d$. Using these relations, we see that

$$
g(t)=\frac{\int_{0}^{\tau} \delta(s+t) d s}{(\delta(\tau)-1) \delta(t)}
$$

Replacing $g$ by $-g$, we get the assertion corresponding to the parentheses.

LemMA 3.4.2. Assume that $\lim _{t \rightarrow \pm \infty} d(t)=1$ (resp. $\left.\lim _{t \rightarrow \pm \infty} d(t)=-1\right)$. Then the solution of a differential equation

$$
\frac{d g(t)}{d t}+d(t) g(t)=1(\text { resp. }-1)
$$

with condition

$$
\lim _{t \rightarrow \pm \infty} g(t)=1
$$

is given by

$$
g(t)=\frac{\int_{-\infty}^{0} \delta(s+t) d s}{\delta(t)}\left(\operatorname{resp} . \frac{\int_{0}^{\infty} \delta(s+t) d s}{\delta(t)}\right) .
$$

Proof. Let us consider the case $\lim _{t \rightarrow \pm \infty} d(t)=1$. Note that

$$
\int_{0}^{\infty} \delta(t) d t=\infty, \quad \int_{-\infty}^{0} \delta(t) d t<\infty
$$


The general solution of $d g / d t+d \cdot g=1$ is given by

$$
g(t)=\left\{c+\int_{0}^{t} \delta(t) d t\right\} / \delta(t)
$$

In order that $g$ satisfies condition $\lim _{t \rightarrow-\infty} g(t)=1$, it is necessary to choose

$$
c=\int_{-\infty}^{0} \delta(t) d t
$$

because $\lim _{t \rightarrow-\infty} \delta(t)=0$. Let $c$ be chosen as above. Since $\lim _{t \rightarrow \infty} \delta(t)=\infty$, $\lim _{t \rightarrow-\infty} \delta(t)=0$, by the theorem of de l'Hospital we see that

$$
\lim _{t \rightarrow \pm \infty} g(t)=1
$$

Furthermore, since $\int_{-\infty}^{t} \delta(t) d t=\int_{-\infty}^{0} \delta(s+t) d s$, we obtain

$$
g(t)=\frac{\int_{-\infty}^{0} \delta(s+t) d s}{\delta(t)} .
$$

Similarly, we can prove the assertion corresponding to the parentheses.

LEMmA 3.4.3. Assume that $\lim _{t \rightarrow-\infty} d(t)=1, \lim _{t \rightarrow \infty} d(t)=-1$. Furthermore, assume that

$$
\int_{-\infty}^{0} \delta(t) d t=\int_{0}^{\infty} \delta(t) d t
$$

Let $k$ be a positive constant. Then the solution of a differential equation

$$
\frac{d g(t)}{d t}+d(t) g(t)=-\sin \left(k^{-1} \int_{0}^{t} \delta(t) d t\right)
$$

with conditions

$$
g>0, \quad \lim _{t \rightarrow \pm \infty} g(t)=1
$$

exists if and only if $k=2 / \pi \int_{0}^{\infty} \delta(t) d t$. If that is the case, the solution is given by

$$
g(t)=\frac{k \cos \left(k^{-1} \int_{0}^{t} \delta(t) d t\right)}{\delta(t)} .
$$

Proof. The general solution is 


$$
g(t)=\frac{c-\int_{0}^{t}\left\{\sin \left(k^{-1} \int_{0}^{t} \delta(t) d t\right)\right\} \delta(t) d t}{\delta(t)} .
$$

Changing the variable by $z=\int_{0}^{t} \delta(t) d t$, we observe that

$$
g(t)=\frac{c-k+k \cos \left(k^{-1} \int_{0}^{t} \delta(t) d t\right)}{\delta(t)} .
$$

On the other hand, by the assumption $\lim _{t \rightarrow \pm \infty} d(t)=\mp 1$, we see that $\lim _{t \rightarrow \pm \infty} \delta(t)=0$. Hence, in order that $\lim _{t \rightarrow \infty} g(t)=1$, it is necessary that

$$
c-k+k \cos \left(k^{-1} \int_{0}^{\infty} \delta(t) d t\right)=0
$$

and

$$
\lim _{t \rightarrow \infty} \frac{-\sin \left(k^{-1} \int_{0}^{t} \delta(t) d t\right)}{d(t)}=1 .
$$

Hence, it is necessary that $k=2 / \pi \int_{0}^{\infty} \delta(t) d t$. Conversely, let $k$ be as above. Put $c=k$. Then, we can easily verify that $g(t)$ satisfies $g>0, \lim _{t \rightarrow \pm \infty} g(t)$ $=1$. This proves our lemma.

3.5. The expression of the multiplier $e^{u}$

Let $Z$ be a vector field on $M$. Suppose that $Z$ can be written

$$
Z=e^{v} X
$$

where $X$ is a $C^{2}$ vector field satisfying conditions (a)-(e) in 3.1, and $v$ is a function of class $C^{2}$ with respect to $X$. Let $\left\{\zeta_{t}\right\}$ be the 1-parameter transformation group generated by $Z$ (cf. Lemma 3.2.1). For $p \in M$, put

$$
\delta(t, p)=\exp \left\{\int_{0}^{t}(\operatorname{div} Z)\left(\zeta_{t}(p)\right) d t\right\} .
$$

Let $M=\Omega \cup \Xi \cup M_{0}$ be the decomposition induced by $X$ as in Proposition 3.1.2. Note that $Z$ induces the same decomposition. Now, let $w: M$ $\rightarrow R$ be a function of class $C^{2}$ with respect to $Z$. Put $Y=e^{w} Z$. In terms of $\left\{\zeta_{t}\right\}, \operatorname{div} Z, w$, we shall study the condition for $Y$ to be elementary with stability exponent 1 . Note that we have a basic relation $\operatorname{div} Y=Z\left(e^{w}\right)$ 
$+e^{w} \operatorname{div} Z$ (cf. Lemma 3.2.1).

LEMMA 3.5.1. In order that

$$
(Y-\operatorname{div} Y) \operatorname{div} Y=-1 \quad \text { on } \Omega \cup \Xi
$$

it is necessary and sufficient that

$$
\operatorname{div} Y=\left\{\begin{array}{lr}
1 & \text { on } \Omega_{+} \cup \Xi_{+}, \\
-1 & \text { on } \Omega_{-} \cup \Xi_{-} .
\end{array}\right.
$$

Proof. Suppose that $(Y-\operatorname{div} Y) \operatorname{div} Y=-1$ on $\Omega \cup \Xi$. Let $p \in \Omega \cup \Xi$. Let $\left\{\psi_{s}\right\}$ be the 1-parameter transformation group generated by $Y$. Consider a function

$$
f(s)=(\operatorname{div} Y)\left(\psi_{s}(p)\right) .
$$

It is obvious that the function $f$ is bounded. Since $\Omega \cup \Xi$ is an invariant set of $\left\{\psi_{s}\right\}$, we get

$$
\frac{d f(s)}{d s}-f^{2}(s)=-1 \quad \text { for all } s .
$$

From Lemma 3.2.6 it follows either that $f(s) \equiv \pm 1$ or that $f$ has the following properties:

$$
-1<f(s)<1 \quad \text { for all } s, \text { and } \lim _{s \rightarrow \pm \infty} f(s)=\mp 1 .
$$

Hence, if $p \in \Omega_{+}$, it is obvious that $f(s) \equiv 1$. If $p \in \Xi_{+}$, by the definition of $\Xi_{+}$, we see that $\lim _{s \rightarrow \infty} f(s)=1$, and hence $f(s) \equiv 1$. Consequently, we conclude that $f(s) \equiv 1$ for $p \in \Omega_{+} \cup \Xi_{+}$, in other words, $\operatorname{div} Y=1$ on $\Omega_{+}$ $\cup \Xi_{+}$. Similarly, we see that $\operatorname{div} Y=-1$ on $\Omega_{-} \cup \Xi_{-}$. Conversely, suppose that $\operatorname{div} Y= \pm 1$ on $\Omega_{ \pm} \cup \Xi_{ \pm}$. Since $\Omega_{ \pm} \cup \Xi_{ \pm}$are invariant by $\left\{\psi_{s}\right\}$, we see that $Y(\operatorname{div} Y)=0$ on $\Omega_{ \pm} \cup \Xi_{ \pm}$, and hence that $(Y-\operatorname{div} Y) \operatorname{div} Y$ $=-1$ on $\Omega \cup \Xi$.

Lemma 3.5.2. Suppose that $Z$ has a periodic trajectory $\gamma=\gamma(t)$ with period $\tau$. If $\gamma$ represents an $\alpha$-limit cycle $\{\gamma\}$, then in order that $\operatorname{div} Y=1$ on $\{r\}$, it is necessary and sufficient that

$$
e^{w(p)}=\frac{\int_{0}^{\tau} \delta(t, p) d t}{\delta(\tau, p)-1} \quad \text { for } p \in\{\gamma\} .
$$

If $\gamma$ represents an $\omega$-limit cycle $\{\gamma\}$, then in order that $\operatorname{div} Y=-1$ on $\{\gamma\}$, 
it is necessary and sufficient that

$$
e^{w(p)}=\frac{\int_{0}^{\tau} \delta(t, p) d t}{1-\delta(\tau, p)} \quad \text { for } p \in\{\gamma\} .
$$

Proof. Fix $p \in\{\gamma\}$. Put

$$
g(t)=e^{w\left(\zeta_{t}(p)\right)}, \quad d(t)=(\operatorname{div} Z)\left(\zeta_{t}(p)\right) .
$$

Note that $g(t), d(t)$ are periodic functions with period $\tau$, and that $\int_{0}^{\tau} d(t) d t$ is positive or negative according as $\{\gamma\}$ is an $\alpha$-limit cycle or $\omega$-limit cycle. Since $\operatorname{div} Y=Z\left(e^{w}\right)+e^{w} \operatorname{div} Z$, we have at once

$$
(\operatorname{div} Y)\left(\zeta_{t}(p)\right)=\frac{d g(t)}{d t}+g(t) d(t) .
$$

Hence, we see that $\operatorname{div} Y=1$ on $\{r\}$ if and only if

$$
1=\frac{d g(t)}{d t}+g(t) d(t)
$$

Applying Lemma 3.4.1, we see that $\operatorname{div} Y=1$ on $\{r\}$ if and only if

$$
g(t)=\frac{\int_{0}^{\tau} \delta(s+t) d s}{(\delta(\tau)-1) \delta(t)}
$$

i.e.,

$$
e^{w\left(\zeta_{t}(p)\right)}=\frac{\int_{0}^{\tau} \delta(s+t, p) d s}{(\delta(\tau, p)-1) \delta(t, p)} .
$$

Using the facts that

$$
\begin{gathered}
\delta(\tau, p)=\delta\left(\tau, \zeta_{t}(p)\right) \quad \text { for all } t, \\
\delta(t+s, p)=\delta(t, p) \delta\left(s, \zeta_{t}(p)\right) \quad \text { for all } s, t,
\end{gathered}
$$

we observe that $e^{w\left(\zeta_{t}(p)\right)}$ can be written

$$
e^{w\left(\zeta_{t}(p)\right)}=\frac{\int_{0}^{\tau} \delta\left(s, \zeta_{t}(p)\right) d s}{\delta\left(\tau, \zeta_{t}(p)\right)-1} .
$$

Replacing $Z$ by $-Z$, we obtain the latter part of our lemma.

Remark. Let $\gamma$ be as in the preceding lemma. Suppose that $\{\gamma\}$ is 
an $\omega$-limit cycle. Let $p \in\{\gamma\}$. Then we have

$$
\frac{\int_{0}^{\tau} \delta(t, p) d t}{1-\delta(\tau, p)}=\int_{0}^{\infty} \delta(t, p) d t
$$

In fact, since $1>\delta(\tau, p)>0$, we observe that

$$
\frac{\int_{0}^{\tau} \delta(t, p) d t}{1-\delta(\tau, p)}=\int_{0}^{\tau} \delta(t, p) d t\left\{1+\delta(\tau, p)+\delta^{2}(\tau, p)+\cdots+\delta^{n}(\tau, p)+\cdots\right\} .
$$

Note that $\delta^{n}(\tau, p) \int_{0}^{\tau} \delta(t, p) d t=\int_{n \tau}^{(n+1) \tau} \delta(t, p) d t$, because $\delta^{n}(\tau, p) \delta(t, p)=\delta(n \tau+t, p)$. Then we get at once the desired formula.

Lemma 3.5.3. Assume that $\operatorname{div} Z= \pm 1$ on $\Omega_{ \pm}$. Suppose that $Z$ has a pathpolygon $\Gamma$. If $\Gamma$ is an $\alpha$-limit pathpolygon, then in order that $\operatorname{div} Y$ $=1$ on $\Gamma$ it is necessary and sufficient that

$$
e^{w(p)}=\int_{-\infty}^{0} \delta(t, p) d t \quad \text { for all } p \in \Gamma
$$

If $\Gamma$ is an $\omega$-limit pathpolygon, then in order that $\operatorname{div} Y=-1$ on $\Gamma$ it is necessary and sufficient that

$$
e^{w(p)}=\int_{0}^{\infty} \delta(t, p) d t \quad \text { for all } p \in \Gamma .
$$

Proof. As in the proof of the previous lemma, let $p \in \Gamma$, and put

$$
g(t)=e^{w\left(\zeta_{t}(p)\right)}, \quad d(t)=(\operatorname{div} Z)\left(\zeta_{t}(p)\right) .
$$

Then we have

$$
(\operatorname{div} Y)\left(\zeta_{t}(p)\right)=\frac{d g(t)}{d t}+g(t) d(t)
$$

Suppose that $\Gamma$ is an $\alpha$-limit pathpolygon. By assumption, we have $\operatorname{div} Z$ (saddle points) $= \pm 1$. From this and the definition of $\Gamma$, it follows that $\lim _{t \rightarrow \pm \infty} d(t)=1$. Now, suppose that $\operatorname{div} Y=1$ on $\Gamma$. Then we have

$$
\frac{d g(t)}{d t}+d(t) g(t)=1
$$

First, let $p$ be a saddle point (in $\Gamma$ ). We contend that 


$$
e^{w(p)}=\int_{-\infty}^{0} \delta(t, p) d t=1
$$

Indeed, it is obvious that $g(t)$ is constant, and $d(t) \equiv 1$. Hence we have $g(t) \equiv 1$, whence $e^{w(p)}=1$. On the other hand, we also have $\delta(t, p)=e^{t}$ and hence $\int_{-\infty}^{0} \delta(t, p) d t=1$. This proves what we want. Next, let $p$ be a regular point (in $\Gamma$ ). By what we have seen, we know that $\lim _{t \rightarrow \pm \infty} g(t)=1$. Then by Lemma 3.4.2, we get

$$
g(t)=\frac{\int_{-\infty}^{0} \delta(s+t, p) d s}{\delta(t, p)} .
$$

Using the fact $\delta(t+s, p)=\delta(t, p) \delta\left(s, \zeta_{t}(p)\right)$, we obtain

$$
e^{w\left(\zeta_{t}(p)\right)}=\int_{-\infty}^{0} \delta\left(s, \zeta_{t}(p)\right) d s .
$$

Conversely, suppose that

$$
e^{w(p)}=\int_{-\infty}^{0} \delta(t, p) d t \quad \text { for all } p \in \Gamma .
$$

Let $p$ be a regular point. Since $\Gamma$ is invariant by $\left\{\zeta_{t}\right\}$, we see that $e^{w}$ satisfies the formula obtained above. Again by Lemma 3.4.2 we see that $d g(t) / d t+d(t) g(t)=1$ and hence that $(\operatorname{div} Y)\left(\zeta_{t}(p)\right)=1$. This proves that $\operatorname{div} Y=1$ on the regular points in $\Gamma$. By the continuity of $\operatorname{div} Y$, we conclude that $\operatorname{div} Y=1$ on $\Gamma$. Replacing $Z$ by $-Z$ yields the latter part of our lemma.

Remark. Let $Z$ be as in the preceding lemma. Let $p \in \Xi_{+}$(or $\Xi_{-}$), and put

$$
g(t)=\int_{-\infty}^{0} \delta\left(s, \zeta_{t}(p)\right) d s\left(\operatorname{resp} \cdot \int_{0}^{\infty} \delta\left(s, \zeta_{t}(p)\right) d s\right)
$$

Then from the proof of the lemma, we have

$$
\begin{gathered}
\frac{d g(t)}{d t}=1-(\operatorname{div} Z)\left(\zeta_{t}(p)\right) \cdot g(t) \\
\left(\operatorname{resp} \cdot \frac{d g(t)}{d t}=-1-(\operatorname{div} Z)\left(\zeta_{t}(p)\right) \cdot g(t)\right) .
\end{gathered}
$$

Similarly we can prove the following. 
Lemma 3.5.4. Assume that $\operatorname{div} Z= \pm 1$ on $\Omega_{ \pm} \cup \Pi_{ \pm}$, where $\Pi_{+}, \Pi_{-}$ denote the unions of $\alpha$-limit pathpolygons, $\omega$-limit pathpolygons respectively. Put $\Pi=\Pi_{+} \cup \Pi_{-}$. Then in order that

$$
\operatorname{div} Y=\left\{\begin{array}{lr}
1 & \text { on } \Omega_{+} \cup E_{+}, \\
-1 & \text { on } \Omega_{-} \cup \Xi_{-},
\end{array}\right.
$$

it is necessary and sufficient that

$$
e^{w(p)}= \begin{cases}1 \quad \text { for } p \in \Omega \cup I I, \\ \int_{-\infty}^{0} \delta(t, p) d t \quad \text { for } p \in \Xi_{+}, \\ \int_{0}^{\infty} \delta(t, p) d t \quad \text { for } p \in \Xi_{-} .\end{cases}
$$

Lemma 3.5.5. Assume that $\operatorname{div} Z= \pm 1$ on $\Omega_{ \pm} \cup \Pi_{ \pm}$(as in the preceding lemma). Let $M_{0}$ be the same set as in Proposition 3.1.2.

(i ) The function $\delta(t, p)$ converges to zero uniformly in the wider sense for $p \in M_{0}$ as $t \rightarrow \pm \infty$. Moreover the infinite integrals

$$
\Delta_{+}(p)=\int_{0}^{\infty} \delta(t, p) d t, \quad \Delta_{-}(p)=\int_{-\infty}^{0} \delta(t, p) d t
$$

converge uniformly in the wider sense for $p \in M_{0}$. Hence $\Delta_{+}(p), \Delta_{-}(p)$ are continuous functions on $M_{0}$.

(ii) Put

$$
\Sigma=\left\{p \in M_{0} \mid \Delta_{+}(p)=\Delta_{-}(p)\right\} .
$$

If $Y$ is elementary with stability exponent 1 , then we have

$$
\Sigma=\{p \in M \mid(\operatorname{div} Y)(p)=0\} .
$$

(iii) Each point $p$ in $M_{0}$ can be written uniquely

$$
p=\zeta_{\tau}(q)
$$

with $q \in \Sigma, \tau \in R$.

(iv) If $Y$ is elementary with stability exponent 1 , then we have

$$
e^{w\left(\zeta_{t}(q)\right)}=\frac{k \cos \left(k^{-1} \int_{0}^{t} \delta(t, q) d t\right)}{\delta(t, q)}
$$

for any $q \in \Sigma$ and any $t \in R$, where $k=2 / \pi \int_{0}^{\infty} \delta(t, q) d t$. Conversely, if $w$ 
satisfies this equality for any $q \in \Sigma$ and any $t \in R$, then we have

$$
(Y-\operatorname{div} Y) \operatorname{div} Y=-1 \quad \text { on } M_{0} \text {. }
$$

Proof of (i). By definition we have

$$
\omega-\lim (p) \subset \Omega_{-} \cup \Pi_{-} \quad \text { for } p \in M_{0} \text {. }
$$

By assumption we have

$$
\operatorname{div} Z=-1 \quad \text { on } \Omega_{-} \cup \Pi_{-} .
$$

Hence for each point $p$ in $M_{0}$, we find a real number $T$ and a neighborhood $U(p)$ of $p$ satisfying the following condition: For any $p^{\prime} \in U(p)$, there are a finite number of intervals $I_{1}, I_{2}, \cdots, I_{r}$ such that the sum of the lengths is less than $T$ and such that

$$
(\operatorname{div} Z)\left(\zeta_{t}\left(p^{\prime}\right)\right)<-\frac{1}{2} \quad \text { for all } t \in[0, \infty)-\bigcup_{i=1}^{r} I_{i}
$$

Hence for each $p \in M_{0}$, there is a neighborhood $U(p)$ of $p$ and a constant such that

$$
\int_{0}^{t}(\operatorname{div} Z)\left(\zeta_{t}\left(p^{\prime}\right)\right) d t<-\frac{1}{2} t+\text { const. for all } t \geqslant 0 \text { and all } p^{\prime} \in U(p) .
$$

Clearly $\delta(t, p)$ converges to zero uniformly in the wider sense for $p \in M_{0}$ as $t \rightarrow \infty$. Moreover this inequality shows that the infinite integral

$$
\int_{0}^{\infty} \delta(t, p) d t=\int_{0}^{\infty}\left\{\exp \int_{0}^{t}(\operatorname{div} Z)\left(\zeta_{t}(p)\right) d t\right\} d t
$$

is uniformly convergent in the wider sense, and hence a continuous function of $p \in M_{0}$. Similarly, we can prove the other assertions.

Proof of (ii). Suppose that $Y$ is elementary with stability exponent 1. Then from Lemma 3.5.1, Propositions 3.2.5 and 3.1.2, we observe that

$$
\{p \in M \mid(\operatorname{div} Y)(p)= \pm 1\}=\Omega_{ \pm} \cup \Xi_{ \pm} .
$$

Hence in particular, we see that if $p$ satisfies $(\operatorname{div} Y)(p)=0$, then $p \in M_{0}$. Therefore to prove (ii), it suffices to prove that for $p \in M_{0}$, the condition $\Delta_{+}(p)=\Delta_{-}(p)$ is equivalent to the condition $(\operatorname{div} Y)(p)=0$. Let $\left\{\psi_{s}\right\}$ be the 1-parameter group generated by $Y$. Let $p \in M_{0}$. Put

$$
\varepsilon(s)=\exp \left\{\int_{0}^{s}(\operatorname{div} Y)\left(\psi_{s}(p)\right) d s\right\} .
$$


Using Lemma 3.2.1, we have

$$
\begin{aligned}
& \Delta_{+}(p)=\int_{0}^{\infty} \delta(t, p) d t=e^{w(p)} \int_{0}^{\infty} \varepsilon(s) d s \\
& \Delta_{-}(p)=\int_{-\infty}^{0} \delta(t, p) d t=e^{w(p)} \int_{-\infty}^{0} \varepsilon(s) d s .
\end{aligned}
$$

On the other hand, by Lemma 3.2.4 we know that the condition $\int_{0}^{\infty} \varepsilon(s) d s$ $=\int_{-\infty}^{0} \varepsilon(s) d s$ is equivalent to the condition $(\operatorname{div} Y)(p)=0$. These prove what we want.

Proof of (iii). Fix $p \in M_{0}$. Consider a function

$$
h(t)=\Delta_{+}\left(\zeta_{t}(p)\right)-\Delta_{-}\left(\zeta_{t}(p)\right) .
$$

Since the condition $h(-\tau)=0$ is equivalent to the condition $\zeta_{-\tau}(p) \in \Sigma$, it suffices to prove that $h(t)$ has a unique zero. First, we shall prove its existence. By the assumption $\operatorname{div} Z= \pm 1$ on $\Omega_{ \pm} \cup \Pi_{ \pm}$and by the definition of $M_{0}$, we have

$$
\lim _{t \rightarrow \pm \infty}(\operatorname{div} Z)\left(\zeta_{t}(p)\right)=\mp 1
$$

From this it follows that

$$
\lim _{t \rightarrow \pm \infty} \Delta_{ \pm}\left(\zeta_{t}(p)\right)=1, \quad \lim _{t \rightarrow \mp \infty} \Delta_{ \pm}\left(\zeta_{t}(p)\right)=\infty .
$$

Hence we obtain

$$
\lim _{t \rightarrow \pm \infty} h(t)=\mp \infty
$$

which shows that $h(t)$ must have at least one zero. Next, to prove the uniqueness, we shall prove that $h(t)$ satisfies

$$
\frac{d h(t)}{d t}=-2-(\operatorname{div} Z)\left(\zeta_{t}(p)\right) h(t)
$$

This will show that the differential coefficients of $h$ at zeros are negative and hence prove the uniqueness of the zero of $h$. Note that

$$
\frac{d}{d t}(\operatorname{div} Z)\left(\zeta_{s}\left(\zeta_{t}(p)\right)\right)=\frac{d}{d s}(\operatorname{div} Z)\left(\zeta_{s}\left(\zeta_{t}(p)\right)\right)
$$

Then 


$$
\frac{d}{d t} \delta\left(s, \zeta_{t}(p)\right)=\frac{d}{d s} \delta\left(s, \zeta_{t}(p)\right)-\delta\left(s, \zeta_{t}(p)\right) \cdot(\operatorname{div} Z)\left(\zeta_{t}(p)\right)
$$

Using this formula and the fact

$$
\delta\left(\infty, \zeta_{t}(p)\right)=\delta\left(-\infty, \zeta_{t}(p)\right)=0,
$$

we get

$$
\begin{aligned}
& \frac{d}{d t} \Delta_{+}\left(\zeta_{t}(p)\right)=-1-(\operatorname{div} Z)\left(\zeta_{t}(p)\right) \Delta_{+}\left(\zeta_{t}(p)\right) \\
& \frac{d}{d t} \Delta_{-}\left(\zeta_{t}(p)\right)=1-(\operatorname{div} Z)\left(\zeta_{t}(p)\right) \Delta_{-}\left(\zeta_{t}(p)\right) .
\end{aligned}
$$

Consequently we obtain

$$
\frac{d h(t)}{d t}=-2-(\operatorname{div} Z)\left(\zeta_{t}(p)\right) h(t)
$$

as desired.

Proof of (iv). Fix $q \in \Sigma$. As in the proof of Lemma 3.5.2, we put

$$
g(t)=e^{w\left(\zeta_{t}(q)\right)}, \quad d(t)=(\operatorname{div} Z)\left(\zeta_{t}(q)\right) .
$$

Then

$$
\frac{d g(t)}{d t}+d(t) g(t)=(\operatorname{div} Y)\left(\zeta_{t}(q)\right)
$$

In order to apply Lemma 3.4.3, note that

$$
\lim _{t \rightarrow \pm \infty} d(t)=\mp 1
$$

because $\operatorname{div} Z= \pm 1$ on $\Omega_{ \pm} \cup \Pi_{ \pm}$. Furthermore, note that

$$
\int_{0}^{\infty} \delta(t, q) d t=\int_{-\infty}^{0} \delta(t, q) d t
$$

because $q \in \Sigma$.

Now, suppose that $Y$ is elementary with stability exponent 1 . Then from Lemmas 3.5.1 and 3.5.4 we see at once that

$$
\lim _{t \rightarrow \pm \infty} g(t)=1 \text {. }
$$

We contend that

$$
(\operatorname{div} Y)\left(\zeta_{t}(q)\right)=-\sin \left(e^{-w(q)} \int_{0}^{t} \delta(t, q) d t\right)
$$


This and Lemma 3.4 .3 will show that

$$
\begin{array}{r}
e^{w(q)}=\frac{2}{\pi} \int_{0}^{\infty} \delta(t, q) d t \\
g(t)=\frac{e^{w(q)} \cos \left(e^{-w(q)} \int_{0}^{t} \delta(t, q) d t\right)}{\delta(t, q)},
\end{array}
$$

and hence will prove the former part of (iv). Now, we must prove the formula above. Let $\left\{\psi_{s}\right\}$ be the 1-parameter group generated by $Y$. Put

$$
\varepsilon(s)=\exp \left\{\int_{0}^{s}(\operatorname{div} Y)\left(\psi_{s}(q)\right) d s\right\} .
$$

Let $S(t)$ be the function defined by

$$
\zeta_{t}(q)=\psi_{S(t)}(q)
$$

Then using Lemma 3.2.1, we obtain

$$
\int_{0}^{t} \delta(t, q) d t=e^{w(q)} \int_{0}^{S(t)} \varepsilon(s) d s
$$

On the other hand, by Lemma 3.2.4 we have

$$
\varepsilon(s)=\frac{1}{\cosh s},
$$

because $(\operatorname{div} Y)(q)=0$. We use the fact

$$
\int_{0}^{s} \frac{1}{\cosh s} d s=\arcsin \tanh s
$$

(This function is known as the Gudermann function gd s.) Then we have

$$
\sin \left(e^{-w(q)} \int_{0}^{t} \delta(t, q) d t\right)=\tanh S(t)
$$

By Lemma 3.2.4 we have also

$$
\tanh s=-(\operatorname{div} Y)\left(\psi_{s}(q)\right) .
$$

Consequently we obtain the desired formula.

Conversely, suppose that

$$
e^{w\left(\zeta_{t}(q)\right)}=\frac{k \cos \left(k^{-1} \int_{0}^{t} \delta(t, q) d t\right)}{\delta(t, q)}
$$


for all $t \in \boldsymbol{R}$. This is written

$$
g(t)=\frac{k \cos \left(k^{-1} \int_{0}^{t} \delta(t) d t\right)}{\delta(t)},
$$

where

$$
\delta(t)=\exp \left(\int_{0}^{t} d(t) d t\right), \quad k=\frac{2}{\pi} \int_{0}^{\infty} \delta(t) d t .
$$

Applying Lemma 3.4.3, we see that $g(t)$ satisfies

$$
\frac{d g(t)}{d t}+d(t) g(t)=-\sin \left(k^{-1} \int_{0}^{t} \delta(t) d t\right) .
$$

Hence we conclude that

$$
(\operatorname{div} Y)\left(\zeta_{t}(q)\right)=-\sin \left(k^{-1} \int_{0}^{t} \delta(t) d t\right)
$$

On the other hand, since $Y=e^{w} Z$, we have

$$
(Y \operatorname{div} Y)\left(\zeta_{t}(q)\right)=e^{w\left(\zeta_{t}(q)\right)} \frac{d}{d t}\left\{(\operatorname{div} Y)\left(\zeta_{t}(q)\right)\right\} .
$$

Consequently,

$$
(Y \operatorname{div} Y)\left(\zeta_{t}(q)\right)=e^{w\left(\zeta_{t}(q)\right)} \frac{d}{d t}\left\{-\sin \left(k^{-1} \int_{0}^{t} \delta(t) d t\right)\right\},
$$

Calculating the derivative of the right-hand side yields

$$
\begin{aligned}
(Y \operatorname{div} Y)\left(\zeta_{t}(q)\right) & =-g(t) k^{-1} \delta(t) \cos \left(k^{-1} \int_{0}^{t} \delta(t) d t\right) \\
& =-\left\{\cos \left(k^{-1} \int_{0}^{t} \delta(t) d t\right)\right\}^{2} \\
& =\left\{(\operatorname{div} Y)\left(\zeta_{t}(q)\right)\right\}^{2}-1 .
\end{aligned}
$$

This and (iii) show that $Y \operatorname{div} Y=(\operatorname{div} Y)^{2}-1$ on $M_{0}$. Our lemma is proved.

Remark. Let the assumption and the notation be as in Lemma 3.5.5. Fix $q \in \Sigma$. Put

$$
g(t)=\frac{k \cos \left(k^{-1} \int_{0}^{t} \delta(t, q) d t\right)}{\delta(t, q)} .
$$


Then we have

$$
\frac{d g(t)}{d t}=-\sin \left(k^{-1} \int_{0}^{t} \delta(t, q) d t\right)-(\operatorname{div} Z)\left(\zeta_{t}(q)\right) g(t) .
$$

\subsection{Proof of Proposition 3.3.2}

Let $X$ be a $C^{2}$ vector field satisfying conditions (a)-(e). We use the notation in Proposition 3.1.2. Let $M=\Omega \cup \Xi \cup M_{0}$ be the decomposition induced by $X$. Let $v: M \rightarrow R$ be a function of class $C^{2}$ with respect to $X$. As in 3.5 , put $Z=e^{v} X$, and let $\left\{\zeta_{t}\right\}$ be the 1-parameter transformation group generated by $Z$. By the following two lemmas we may assume that $Z$ satisfies

$$
\operatorname{div} Z= \pm 1 \quad \text { on } \Omega_{ \pm} \cup \Pi_{ \pm},
$$

and we see that in order to prove our Proposition, it suffices to prove our assertion for this vector field $Z$.

Lemma 3.6.1. There exists a function $v: M \rightarrow R$ of class $C^{2}$ with respect to $X$ such that $\operatorname{div}\left(e^{v} X\right)= \pm 1$ on $\Omega_{ \pm} \cup \Pi_{ \pm}$.

This lemma will be proved in 3.7.

Lemma 3.6.2. Let $v$ be a function of class $C^{2}$ with respect to $X$. Put $Z=e^{v} X$. If there exists uniquely a function $w: M \rightarrow R$ of class $C^{2}$ with respect to $Z$ such that $e^{w} Z$ is elementary with stability exponent 1 , then there exists uniquely a function $u: M \rightarrow R$ satisfying the conditions (i), (ii) in Proposition 3.3.2.

Proof. We can check directly that the function $u=v+w$ has the desired property.

Now, we return to the proof of our proposition. Note that we can use Lemmas 3.5.4 and 3.5.5, because we have assumed that $\operatorname{div} Z= \pm 1$ on $\Omega_{ \pm} \cup \Pi_{ \pm}$. First, we shall prove the uniqueness of such a function $w$.

Suppose that there is a function $w$ of class $C^{2}$ with respect to $Z$ such that $e^{w} Z$ is elementary with stability exponent 1. From Lemmas 3.5.1 and 3.5.4, we have

$$
e^{w(p)}= \begin{cases}1 \quad \text { for } p \in \Omega \cup \Pi, \\ \int_{-\infty}^{0} \delta(t, p) d t & \text { for } p \in \Xi_{+}, \\ \int_{0}^{\infty} \delta(t, p) d t & \text { for } p \in \Xi_{-},\end{cases}
$$


where

$$
\delta(t, p)=\exp \left\{\int_{0}^{t}(\operatorname{div} Z)\left(\zeta_{t}(p)\right) d t\right\} .
$$

Furthermore, by Lemma 3.5.5 we have

$$
e^{w(p)}=\frac{k \cos \left(k^{-1} \int_{0}^{\tau} \delta(t, q) d t\right)}{\delta(\tau, q)} \quad \text { for } p=\zeta_{\tau}(q) \in M_{0},
$$

where

$$
q \in \Sigma=\left\{p \in M_{0} \mid \int_{0}^{\infty} \delta(t, p) d t=\int_{-\infty}^{0} \delta(t, p) d t\right\}, \quad k=\frac{2}{\pi} \int_{0}^{\infty} \delta(t, q) d t .
$$

Since $M=\Omega \cup \Xi \cup M_{0}$, these expressions show that $w$ is uniquely determined (provided it exists).

We shall now prove its existence. Define $w: M \rightarrow R$ by the expressions obtained above. It is well-defined, because if $p \in \Pi_{+}$(or $\Pi_{-}$), then by our assumption $\operatorname{div} Z= \pm 1$ on $\Pi_{ \pm}$, we have

$$
\int_{-\infty}^{0} \delta(t, p) d t=1 \quad\left(\text { or } \int_{0}^{\infty} \delta(t, p) d t=1\right) .
$$

If we knew that this function $w$ is of class $C^{2}$ with respect to $Z$, then again by Lemmas 3.5.1, 3.5.4, and 3.5.5 we could conclude that the vector field $Y=e^{w} Z$ satisfies $(Y-\operatorname{div} Y) \operatorname{div} Y=-1$ on $M$, in other words, $Y$ is elementary with stability exponent 1 . Therefore, to prove our proposition it suffices to prove that $w$ is of class $C^{2}$ with respect to $Z$. For this it suffices to prove that it is true for $e^{w}$.

First, we must verify the continuity of $e^{w}$. From the continuity of the function $\operatorname{div} Z$, it follows at once that

$$
\delta(t, q)=\exp \left\{\int_{0}^{t}(\operatorname{div} Z)\left(\zeta_{t}(q)\right) d t\right\}
$$

is a continuous function of $t, q$. By (i), Lemma 3.5.5 we know that $k=$ $\int_{0}^{\infty} \delta(t, q) d t$ is also a continuous function of $q \in \Sigma$. Consequently, we see that

$$
\frac{k \cos \left(k^{-1} \int_{0}^{t} \delta(t, q) d t\right)}{\delta(t, q)}
$$


is a continuous function of $t, q$, in other words, the function $e^{w}$ is continuous on the open set $M_{0}$. Let $p \in \Omega_{-} \cup E_{\text {. }}$. To prove the continuity of $e^{w}$ at $p$, let $\left\{p_{n}\right\}$ be any sequence convergent to $p$. We shall prove $\lim _{n \rightarrow \infty} e^{w\left(p_{n}\right)}=e^{w(p)}$. Note that a sufficiently small neighborhood of $p$ is contained in $\Omega_{-} \cup \Xi_{-} \cup M_{0}$, because $\Omega_{+} \cup \Xi_{+}$is closed. Therefore to prove $\lim _{n \rightarrow \infty} e^{w\left(p_{n}\right)}=e^{w(p)}$, it suffices to prove this in the case where either the sequence $\left\{p_{n}\right\}$ is contained in $\Omega_{-} \cup \Xi_{-}$, or $\left\{p_{n}\right\}$ is contained in $M_{0}$. Note that we can write

$$
e^{w\left(p^{\prime}\right)}=\int_{0}^{\infty} \delta\left(t, p^{\prime}\right) d t \quad \text { for } p^{\prime} \in \Omega_{-} \cup \Xi_{-}
$$

because $\int_{0}^{\infty} \delta\left(t, p^{\prime}\right) d t=1$ for $p^{\prime} \in \Omega_{-}$. ' On the other hand, from the proof of (i), Lemma 3.5.5 we have:

Lemma 3.6.3. The function $\Delta_{+}: \Omega_{-} \cup \Xi_{-} \cup M_{0} \rightarrow R$ defined by

$$
\Delta_{+}(p)=\int_{0}^{\infty} \delta(t, p) d t
$$

is continuous.

Hence, if the sequence $\left\{p_{n}\right\}$ is contained in $\Omega_{-} \cup \Xi_{-}$, then we have

$$
\lim _{n \rightarrow \infty} e^{w\left(p_{n}\right)}=\lim _{n \rightarrow \infty} \Delta_{+}\left(p_{n}\right)=\Delta_{+}(p)=e^{w(p)},
$$

as desired. Suppose now that $\left\{p_{n}\right\}$ is contained in $M_{0}$. Then by (iii), Lemma 3.5.5 we can write

$$
p_{n}=\zeta_{\tau_{n}}\left(q_{n}\right) \quad \text { with } q_{n} \in \Sigma
$$

Note that $\tau_{n} \rightarrow \infty$ as $n \rightarrow \infty$, because $p \notin M_{0}$. Consider the inequality

$$
\left|e^{w\left(p_{n}\right)}-e^{w(p)}\right| \leqslant\left|e^{w\left(p_{n}\right)}-\int_{0}^{\infty} \delta\left(t, p_{n}\right) d t\right|+\left|\int_{0}^{\infty} \delta\left(t, p_{n}\right) d t-\int_{0}^{\infty} \delta(t, p) d t\right| .
$$

From Lemma 3.6.3 it follows that the second term in the right-hand side converges to zero as $p_{n} \rightarrow p$. By the following lemma, we see that the first term in the right-hand side also converges to zero as $p_{n} \rightarrow p$, and hence we conclude that $e^{w}$ is continuous at any point in $\Omega_{-} \cup \Xi_{-}$.

LEMmA 3.6.4. There exist two positive numbers $c, T$ such that

$$
\left|e^{w\left(\zeta_{\tau}(q)\right)}-\int_{0}^{\infty} \delta\left(t, \zeta_{\tau}(q)\right) d t\right|<c\left|1-\int_{0}^{\tau} \delta(t, q) d t / \int_{0}^{\infty} \delta(t, q) d t\right|
$$


for any $q \in \Sigma$ and any $\tau \geqslant T$.

Proof. From the relation $\delta(\tau+t, q)=\delta(\tau, q) \delta\left(t, \zeta_{\tau}(q)\right)$, we have

$$
\int_{0}^{\infty} \delta\left(t, \zeta_{\tau}(q)\right) d t=\frac{\frac{\pi}{2} k-\int_{0}^{\tau} \delta(t, q) d t}{\delta(\tau, q)}
$$

Hence

$$
\begin{aligned}
& \left|e^{w\left(\zeta_{\tau}(q)\right)}-\int_{0}^{\infty} \delta\left(t, \zeta_{\tau}(q)\right) d t\right| \\
& =\frac{k}{\delta(\tau, q)}\left|\cos \left(k^{-1} \int_{0}^{\tau} \delta(t, q) d t\right)-\left(\frac{\pi}{2}-k^{-1} \int_{0}^{\tau} \delta(t, q) d t\right)\right| .
\end{aligned}
$$

Using the estimate

$$
\left|\cos x-\left(\frac{\pi}{2}-x\right)\right| \leqslant \text { const. }\left(x-\frac{\pi}{2}\right)^{2} \quad \text { for } x \text { close to } \frac{\pi}{2},
$$

we obtain

$$
\left|e^{w\left(\zeta_{\tau}(q)\right)}-\int_{0}^{\infty} \delta\left(t, \zeta_{\tau}(q)\right) d t\right| \leqslant \frac{\text { const. }}{\delta(\tau, q)} k\left(\frac{\pi}{2}-k^{-1} \int_{0}^{\tau} \delta(t, q) d t\right)^{2}
$$

if $\left|\pi / 2-k^{-1} \int_{0}^{\tau} \delta(t, q) d t\right|$ is small. From (i), Lemma 3.5.5 and the compactness of $\Sigma$, it follows that $\pi / 2-k^{-1} \int_{0}^{t} \delta(t, q) d t$ converges to zero uniformly for $q$ in $\Sigma$ as $\tau \rightarrow \infty$. The right-hand side of the inequality obtained above is written

$$
\text { const. }\left(\frac{\pi}{2}\right)^{2} k\left(1-\frac{\int_{0}^{\tau} \delta(t, q) d t}{\int_{0}^{\infty} \delta(t, q) d t}\right)\left(1-\frac{\int_{0}^{\tau} \delta(t, q) d t}{\int_{0}^{\infty} \delta(t, q) d t}\right)\left(\frac{1}{\delta(\tau, q)}\right) .
$$

From the continuity of the function $k$ on $\Sigma$, it follows that $k$ is bounded. Therefore, to prove our lemma it suffices to prove that the function

$$
\left(1-\frac{\int_{0}^{\tau} \delta(t, q) d t}{\int_{0}^{\infty} \delta(t, q) d t}\right)\left(\frac{1}{\delta(\tau, q)}\right)
$$

is bounded on $\{(\tau, q) \mid \tau \geqslant 0, q \in \Sigma\}$. Note that this function is equal to 


$$
\frac{\Delta_{+}\left(\zeta_{\tau}(q)\right)}{\int_{0}^{\infty} \delta(t, q) d t}
$$

Since $\Delta_{+}$is continuous on $\Omega_{-} \cup \Xi_{-} \cup M_{0}$ (Lemma 3.6.3), it is obvious that $\Delta_{+}$is bounded on the subset $\left\{\zeta_{t}(q) \mid t \geqslant 0, q \in \Sigma\right\}$. From this and the fact that the function $k$ is positive and bounded below, we see that the function $\Delta_{+}\left(\zeta_{\tau}(q)\right)\left(\int_{0}^{\infty} \delta(t, q) d t\right)^{-1}$ is bounded on $\{(\tau, q) \mid \tau \geqslant 0, q \in \Sigma\}$. This proves our lemma.

Similarly, we see that $e^{w}$ is continuous at any point in $\Omega_{+} \cup \Xi_{+}$. Consequently we know that $e^{w}$ is continuous on the whole $M$.

Next, to study $Z\left(e^{w}\right)$ we use Remarks following Lemmas 3.5.3 and 3.5.5. Then, noting that $\operatorname{div} Z= \pm 1, e^{w}=1$ on $\Omega_{ \pm}$, we have

$$
\left(Z e^{w}\right)(p)=\left\{\begin{array}{l} 
\pm 1-(\operatorname{div} Z)(p) e^{w(p)} \quad \text { for } p \in \Omega_{ \pm} \cup \Xi_{ \pm}, \\
-\sin \left(k^{-1} \int_{0}^{\tau} \delta(t, q) d t\right)-(\operatorname{div} Z)(p) e^{w(p)} \\
\quad \text { for } p=\zeta_{\tau}(q) \in M_{0}, q \in \Sigma .
\end{array}\right.
$$

Note that $\operatorname{div} Z$ is continuous on $M$. Furthermore we know already the continuity of $e^{w}$. Therefore to prove the continuity of the function $Z\left(e^{w}\right)$ on $M$, it suffices to prove that the function $S$ defined by

$$
S(p)=\left\{\begin{array}{l} 
\pm 1 \quad \text { for } p \in \Omega_{ \pm} \cup \Xi_{ \pm}, \\
-\sin \left(k^{-1} \int_{0}^{\tau} \delta(t, q) d t\right) \quad \text { for } p=\zeta_{\tau}(q) \in M_{0}, q \in \Sigma
\end{array}\right.
$$

is continuous on $M$. This is an immediate consequence of the fact that $k^{-1} \int_{0}^{\tau} \delta(t, q) d t$ converges to $\pm \pi / 2$ uniformly for $q$ in $\Sigma$ as $\tau \rightarrow \pm \infty$. Consequently, the continuity of $Z\left(e^{w}\right)$ is proved.

Finally, to study $Z^{2}\left(e^{w}\right)$, note that the function $(\operatorname{div} Z) e^{w}$ is of class $C^{1}$ with respect to $Z$, because it is true for $\operatorname{div} Z, e^{w}$. Therefore, to prove that $Z\left(e^{w}\right)$ is of class $C^{1}$ with respect to $Z$, it suffices to prove that the function $S$ given above is of class $C^{1}$ with respect to $Z$. It is obvious that

$$
(Z S)(p)=\left\{\begin{array}{l}
0 \quad \text { for } p \in \Omega \cup \Xi, \\
-k^{-1} \delta(\tau, q) \cos \left(k^{-1} \int_{0}^{\tau} \delta(t, q) d t\right) \quad \text { for } p=\zeta_{\tau}(q) \in M_{0}, q \in \Sigma .
\end{array}\right.
$$

This function $Z S$ is certainly continuous, because $\delta(\tau, q)$ converges to zero uniformly for $q$ in $\Sigma$ as $\tau \rightarrow \pm \infty$ (Lemma 3.5.5, (i)). Consequently, the 
function $Z\left(e^{w}\right)$ is of class $C^{1}$ with respect to $Z$, and hence our function $e^{w}$ is of class $C^{2}$ with respect to $Z$. This completes the proof of our proposition.

\subsection{Proof of Lemma 3.6.1}

Let $X$ be a $C^{2}$ vector field satisfying conditions (a)-(e). Let $\left\{\phi_{t}\right\}$ be the 1-parameter transformation group generated by $X$. Using Remark 2 in 3.1 and Lemma 3.5.2, we obtain easily a function $v: M \rightarrow R$ of class $C^{2}$ such that $\operatorname{div}\left(e^{v} X\right)= \pm 1$ on $\Omega_{ \pm}$. Because $\Omega$ consists of a finite number of points and a finite number of circles (Proposition 3.1.2). Using Lemma 3.5.3, we can modify the function $v$ so that

(i) $v$ is continuous on $M$.

(ii) $v$ is of class $C^{2}$ on $M-U$, where $U$ is a neighborhood of $\Pi \cap$ \{saddle points\}.

(iii) $X v, X^{2} v$ exist on $\Pi \cap U$ and are continuous on it.

(iv) $\operatorname{div}\left(e^{v} X\right)= \pm 1$ on $\Omega_{ \pm} \cup \Pi_{ \pm}$.

Furthermore, we must modify $v$ so that $v$ is of class $C^{2}$ with respect to $X$. For this purpose we need a lemma. Note that for each saddle point $p$ of $X$, there exists a local coordinate system $(x, y)$ of class $C^{2}$, in a coordinate neighborhood $V$ of $p$, such that $\left.X\right|_{V}$ is written in the form

$$
\left.X\right|_{V}=a(x, y) x \frac{\partial}{\partial x}+b(x, y) y \frac{\partial}{\partial y},
$$

where $a(x, y), b(x, y)$ are of class $C^{1}$ and satisfy $a(x, y)>0, b(x, y)<0$.

LEMMA 3.7.1. Let $X$ be a $C^{2}$ vector field on a neighborhood of the origin of $\boldsymbol{R}^{2}$, and assume that $X$ is written in the form

$$
X=a(x, y) x \frac{\partial}{\partial x}+b(x, y) y \frac{\partial}{\partial y},
$$

where $a(x, y), b(x, y)$ are of class $C^{1}$ and nonzero. Let $f(x), g(y)$ be functions of $x, y$ respectively. Assume that $f(x), g(y)$ are of class $C^{2}$ with respect to $a(x, 0) x \partial / \partial x, b(0, y) y \partial / \partial y$ respectively, and assume that $f(0)=g(0)$. Put

$$
h(x, y)=f(x)+g(y)-f(0) .
$$

Then the function $h(x, y)$ is of class $C^{2}$ with respect to $X$, and its restrictions to the $x$-axis, $y$-axis coincide with $f(x), g(y)$ respectively. Furthermore 
$h(x, y)$ is of class $C^{2}$ on $\{(x, y) \mid x \neq 0, y \neq 0\}$.

Proof. This can be directly checked.

Using this lemma, we obtain at once a function $v$ having the desired property. Lemma 3.6.1 is proved.

\subsection{Proof of Theorem 1.1 .2}

Suppose that a $C^{2}$ vector field $X$ is structurally stable. Then the characterization theorem of Peixoto ([10]) asserts that $X$ satisfies conditions (a), (c), (d), (e) in 3.1. Suppose that $X$ satisfies condition (b) besides. Then we can apply Theorem 3.3.1. Hence we find a function $u: M \rightarrow \boldsymbol{R}$ satisfying the conditions (i)-(iii) in Theorem 3.3.1. Since $e^{u} X$ is elementary with stability exponent $\lambda$, from Lemma 3.2.2 it follows that $u$ satisfies equation $(\mathrm{E})$ in Theorem 1.1.2. Our theorem is proved.

\section{REFERENCES}

[1] L. A. Cherkas, Estimation of the number of limit cycles of autonomous systems, Differential Equations 13 (1977), 529-547, (transl. from the Russian).

[2] C. Comstock, On the limit cycles of $y^{\prime \prime}+\mu F^{\prime}\left(y^{\prime}\right)+y=0$, J. Differential Equations 8 (1970), 173-179.

[ 3 ] R. N. D'heedene, For all real $\mu, \ddot{x}+\mu \sin \dot{x}+x=0$ has an infinite number of limit cycles, J. Differential Equations 5 (1969), 564-571.

[4] J. R. Graef, On the generalized Liénard equation with negative damping, J. Differential Equations 12 (1972), 34-62.

[5] M. W. Hirsch and S. Smale, Differential Equations, Dynamical Systems, and Linear Algebra, Academic Press, 1974.

[ 6 ] H. Hochstadt and B. H. Stephan, On the limit cycles of $\ddot{x}+\mu \sin \dot{x}+x=0$, Arch. Rational Mech. Anal. 23 (1967), 369-379.

[ 7 ] S. Kobayashi and K. Nomizu, Foundations of Differential Geometry I, Interscience Publishers, 1963.

[ 8 ] J. La Salle and S. Lefschetz, Stability by Liapunov's Direct Method with Applications, Academic Press, 1961.

[9] S. Lefschetz, Differential Equations: Geometric Theory, 2nd ed., Interscience Publishers.

[10] M. M. Peixoto, Structural stability on two-dimensional manifolds, Topology 1 (1962), 101-120.

[11] P. J. Ponzo and N. Wax, On periodic solutions of the system $\dot{x}=y-F(x), \dot{y}=$ - $g(x)$, J. Differential Equations 10 (1971), 262-269.

[12] K. Yamato, Qualitative theory of codimension-one foliations, Nagoya Math. J., 49 (1973), 155-229. 
[13] R. Bellman, Methods of Nonlinear Analysis, Academic Press, Vol. 1 (1970), Vol. 2 (1973).

Department of Mathematics College of General Education Nagoya University, 